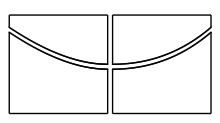

\author{
Universidade de Brasília \\ Instituto de Ciências Exatas \\ Departamento de Estatística
}

Dissertação de Mestrado

Estimação não paramétrica da distribuição do tempo de falha para dados de status corrente com erros de classificação

por

Augusto de Araújo Maia

Orientador: Prof. Dr. Antonio Eduardo Gomes

Junho de 2015 


\title{
Estimação não paramétrica da distribuição do tempo de falha para dados de status corrente com erros de classificação
}

\author{
Dissertação apresentada ao Departamento de \\ Estatística do Instituto de Ciências Exatas \\ da Universidadede de Brasília como requisito \\ parcial à obtenção do título de Mestre em \\ Estatística.
}

Universidade de Brasília

Brasília, Junho de 2015 
Termo de Aprovação

Augusto de Araújo Maia

\section{EstimaÇÃo NÃo PARAMÉtriCA DA DISTRIBUiÇÃO DO TEMPO DE FALHA PARA DADOS DE STATUS CORRENTE COM ERROS DE CLASSIFICAÇÃO}

Dissertação apresentada ao Departamento de Estatística do Instituto de Ciências Exatas da Universidadede de Brasília como requisito parcial à obtenção do título de Mestre em Estatística.

Data da defesa: 19 de Junho de 2015

Orientador:

Prof. Dr. Antonio Eduardo Gomes

Departamento de Estatística, UnB

Comissão Examinadora:

Prof. Dr. Eduardo Yoshio Nakano

Departamento de Estatística, UnB

Prof. Dra. Linda Lee Ho Departamento de Engenharia de Produção, USP

Brasília, Junho de 2015 
Ficha Catalográfica

DE ARAÚJO MAIA, AUGUSTO

Estimação não paramétrica da distribuição do tempo de falha para dados de status corrente com erros de classificação,

(UnB - IE, Mestre em Estatística, 2015).

Dissertação de Mestrado - Universidade de Brasília. Departamento de Estatística

- Instituto de Ciências Exatas.

1. estimação não-paramétrica de máxima verossimilhança 2. dados de status corrente 3. erros de classificação

É concedida à Universidade de Brasília a permissão para reproduzir cópias desta dissertação de mestrado e para emprestar ou vender tais cópias somente para propósitos acadêmicos e científicos. O autor reserva outros direitos de publicação e nenhuma parte desta monografia de Projeto Final pode ser reproduzida sem a autorização por escrito do autor.

Augusto de Araújo Maia 
Dedico esta obra a minha amada esposa, aos meus pais, a meu irmão e aos meus amigos. 


\section{Agradecimentos}

- Agradeço ao Supremo Deus e ao Messias Meishu-Sama pela graça de poder fazer um mestrado;

- agradeço a Eles por todos os anjos, pessoas maravilhosas, que estiveram comigo nesta jornada, me apoiando e me ajudando de alguma forma;

- agradeço a minha família e a meus amigos maravilhosos

- e, finalmente, agradeço a meu grande orientador professor Antonio Eduardo, pessoa pela qual tenho profunda admiração, respeito e eterna gratidão.

"Quando vejo alguém se empenhando pelo bem do próximo e do mundo, tenho a sensação de estar vendo um diamante entre o cascalho." Meishu-Sama (1953) 


\section{Sumário}

$\begin{array}{ll}\text { Lista de Figuras } & 4\end{array}$

$\begin{array}{ll}\text { Lista de Tabelas } & 6\end{array}$

$\begin{array}{ll}\text { Resumo } & 7\end{array}$

$\begin{array}{lr}\text { Abstract } & 8\end{array}$

1 Conceitos Básicos em Análise de Sobrevivência 10

1.1 Conceitos . . . . . . . . . . . . . . . . . . . . 10

1.1.1 Censura ......................... 10

1.1.2 Função de distribuição acumulada e funções relacionadas . . . 11

1.2 Estimação da função de sobrevivência . . . . . . . . . . . . . . . . . . 15

1.2.1 Estimação paramétrica . . . . . . . . . . . . . . . 15

1.2.2 Estimação não paramétrica da função de sobrevivência . . . 18

1.3 Modelo de regressão de Cox . . . . . . . . . . . . . . . . . . . . . . . 19

2 Dados de status corrente com erro de classificação 21

2.1 Conceitos de sensibilidade e especificidade . . . . . . . . . . . 22

2.2 Estimador não-paramétrico de máxima verossi-milhança . . . . . . . 23

2.2.1 Otimização Convexa . . . . . . . . . . . . . . . . . 23

2.2.2 Interpretação Gráfica: função minorante convexa máxima . . . 29

2.2 .3 A fórmula max-min . . . . . . . . . . . . . . . . . . 31

2.2.4 O Algoritmo Pool-Adjacent-Violators(PAV) . . . . . . . . . . 32

2.2.5 ENPMV sem erro de classificação para dados de status corrente 33

2.3 Métodos para correção do ENPMV com erros de classificação . . . . . 36 
2.3.1 ENPMV com erro de classificação . . . . . . . . . . . . . . 36

3 Núcleo-estimadores $\quad 46$

3.1 Motivação para o método núcleo-estimadores . . . . . . . . . . . . . . 46

3.1.1 Estimação de densidades pelo método de núcleo-estima-dores (kernel) . . . . . . . . . . . . . . . . 4 46

3.1 .2 Histogramas . . . . . . . . . . . . . . . . 47

3.1.3 Estimação de Densidades Univariadas pelo Método Kernel . . 49

3.1.4 Estimadores de função peso geral . . . . . . . . . . . . . . . 51

3.2 Medidas de discrepância: erro quadrático médio e o erro quadrático médio integrado . . . . . . . . . . . . . . . . . 52

3.2.1 Propriedades elementares de uma amostra infinita . . . . . . . 53

3.3 Suavização do ENPMV da função de distribuição acumulada do tempo de falha para dados de status corrente . . . . . . . . . . 54

4 Simulação e análise dos resultados $\quad 55$

4.1 Integração com eliminação de parâmetros incovenientes . . . . . . . . 55

4.2 Simulação e análise dos resultados . . . . . . . . . . . . . . . . . 56

4.2.1 Avaliação do vício em função da sensibilidade e especificidade 59

4.2.2 Versões suavizadas da função de distribuição . . . . . . . . . . 62

4.2.3 Aplicação para um conjunto de dados reais . . . . . . . . . . . 63

4.3 Conclusões . . . . . . . . . . . . . . . . . . . . 65

4.4 Trabalhos Futuros . . . . . . . . . . . . . . . . 66

$\begin{array}{ll}\text { Referências Bibliográficas } & 67\end{array}$ 


\section{Lista de Figuras}

1.1 Função de sobrevivência da Weibull para $\alpha=0.5, \lambda=0.26328(-)$; $\alpha=1.0, \lambda=0.1(\ldots \ldots) ; \alpha=3.0, \lambda=0.00208(\ldots \ldots) \ldots \ldots$

1.2 Função de Sobrevivência para o tempo de vida de uma variável aleatória discreta . . . . . . . . . . . . . . . . . . . . . 14

2.1 Diagrama das Somas Acumuladas (DSA) e a função Minorante Convexa Máxima $(\mathrm{MCM}) \ldots . . \ldots 30$

2.2 Diagrama das Somas Acumuladas e a Minorante Convexa Máxima para os dados da Tabela $2.1 \ldots 31$

2.3 Interpretação gráfica do pooling-adjacent-violators . . . . . . . . . . . 32

2.4 Diagrama das Somas Acumuladas (para os pontos $\left(W_{i}, G_{i}\right)$ ) e sua função minorante convexa máxima . . . . . . . . . . . . . . . 35

2.5 Hipotético ENPMV sem restrição, com as posições hipotéticas $s$ e $1-e$ mostradas no eixo vertical e os pontos $k_{0}, k_{1}, m_{0}$ e $m_{1}$ mostrados no eixo

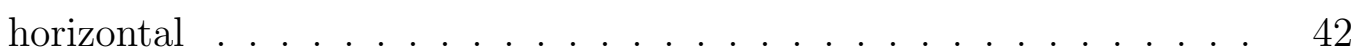

2.6 Diagrama das estimativas não paramétricas para os métodos propostos 44

2.7 Diagrama das estimativas paramétricas para os métodos propostos . . 45

3.1 Estimativa da densidade univariada pelo método kernel, $(-)$ densidade estimada e (- - - ) funções kernel individuais. . . . . . . . . . . 51

4.1 Gráfico da média das estimativas a partir do uso de distribuições a priori para sensibilidade e especificidade assumidas . . . . . . . 57

4.2 Gráfico da função de distribuição do tempo de falha, com $50 \%$ de censura à esquerda e $50 \%$ de censura à direita . . . . . . . . . . . 
4.3 Gráfico do vício em função da sensibilidade e especificidade, avaliado no $1^{\circ}$ decil, com $50 \%$ de censura à esquerda e 50\% de censura à direita 60

4.4 Gráfico do vício em função da sensibilidade e especificidade, avaliado no $1^{\circ}$ quartil, com $50 \%$ de censura à esquerda e $50 \%$ de censura à direita 60

4.5 Gráfico do vicio em função da sensibilidade e especificidade, avaliado na mediana, com 50\% de censura à esquerda e 50\% de censura à direita 61

4.6 Gráfico do vício em função da sensibilidade e especificidade, avaliado no $3^{\circ}$ quartil, com $50 \%$ de censura à esquerda e 50\% de censura à direita 62

4.7 Gráfico ilustrativo para estimativas suavizadas da função de distribui-

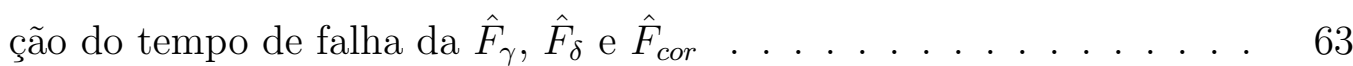

4.8 Gráfico da função suavizada de distribuição estimada do tempo de ocorrência da doença arterial . . . . . . . . . . . . . . . . . . 64 


\section{Lista de Tabelas}

2.1 Comprimento de 42 garotas de uma escola em diferentes idades . . . 24

2.2 Cálculo da log-verossimilhança corrigida, com erro de classificação e sem erro de classificação para a distribuição Weibull . . . . . . . . . . 45

3.1 Funções kernel comumente utilizadas com dados univariados . . . . . 50

4.1 Estimação da esperança e erro-padrão de $F_{\gamma}, F_{\delta}$ e $F_{c o r}$ no quantil da T, SEM suavização, com 50\% de censura à esquerda e 50\% à direita . 71

4.2 Estimação da esperança e erro-padrão de $F_{\gamma}, F_{\delta}$ e $F_{c o r}$ no quantil da T, SEM suavização, com $50 \%$ de censura à esquerda e $50 \%$ à direita .

4.3 Estimação da esperança e erro-padrão de $F_{\gamma}, F_{\delta}$ e $F_{c o r}$ no quantil da T, SEM suavização, com 50\% de censura à esquerda e 50\% à direita . 73

4.4 Estimação da esperança e erro-padrão de $F_{\gamma}, F_{\delta}$ e $F_{c o r}$ no quantil da T, COM suavização, com 50\% de censura à esquerda e 50\% à direita

4.5 Estimação da esperança e erro-padrão de $F_{\gamma}, F_{\delta}$ e $F_{c o r}$ no quantil da T, COM suavização, com $50 \%$ de censura à esquerda e $50 \%$ à direita

4.6 Estimação da esperança e erro-padrão de $F_{\gamma}, F_{\delta}$ e $F_{c o r}$ no quantil da T, COM suavização, com 50\% de censura à esquerda e $50 \%$ à direita

4.7 Estimação da esperança e erro-padrão de $F_{\gamma}, F_{\delta}$ e $F_{c o r}$ no quantil da T, SEM suavização, com $70 \%$ de censura à esquerda e $30 \%$ à direita .

4.8 Estimação da esperança e erro-padrão de $F_{\gamma}, F_{\delta}$ e $F_{c o r}$ no quantil da T, SEM suavização, com $70 \%$ de censura à esquerda e $30 \%$ à direita .

4.9 Estimação da esperança e erro-padrão de $F_{\gamma}, F_{\delta}$ e $F_{c o r}$ no quantil da T, SEM suavização, com $70 \%$ de censura à esquerda e $30 \%$ à direita . 
4.10 Estimação da esperança e erro-padrão de $F_{\gamma}, F_{\delta}$ e $F_{c o r}$ no quantil da T, COM suavização, com $70 \%$ de censura à esquerda e $30 \%$ à direita

4.11 Estimação da esperança e erro-padrão de $F_{\gamma}, F_{\delta}$ e $F_{c o r}$ no quantil da T, COM suavização, com $70 \%$ de censura à esquerda e $30 \%$ à direita

4.12 Estimação da esperança e erro-padrão de $F_{\gamma}, F_{\delta}$ e $F_{c o r}$ no quantil da T, COM suavização, com $70 \%$ de censura à esquerda e $30 \%$ à direita

4.13 Estimação da esperança e erro-padrão de $F_{\gamma}, F_{\delta}$ e $F_{c o r}$ no quantil da T, SEM suavização, com $30 \%$ de censura à esquerda e $70 \%$ à direita .

4.14 Estimação da esperança e erro-padrão de $F_{\gamma}, F_{\delta}$ e $F_{c o r}$ no quantil da T, SEM suavização, com $30 \%$ de censura à esquerda e $70 \%$ à direita .

4.15 Estimação da esperança e erro-padrão de $F_{\gamma}, F_{\delta}$ e $F_{c o r}$ no quantil da T, SEM suavização, com $30 \%$ de censura à esquerda e $70 \%$ à direita .

4.16 Estimação da esperança e erro-padrão de $F_{\gamma}, F_{\delta}$ e $F_{c o r}$ no quantil da T, COM suavização, com $30 \%$ de censura à esquerda e $70 \%$ à direita

4.17 Estimação da esperança e erro-padrão de $F_{\gamma}, F_{\delta}$ e $F_{c o r}$ no quantil da T, COM suavização, com $30 \%$ de censura à esquerda e $70 \%$ à direita

4.18 Estimação da esperança e erro-padrão de $F_{\gamma}, F_{\delta}$ e $F_{c o r}$ no quantil da T, COM suavização, com $30 \%$ de censura à esquerda e $70 \%$ à direita 


\section{Resumo}

Neste estudo, avaliamos o desempenho de um método para correção do estimador não paramétrico de máxima verossimilhança da distribuição do tempo de falha para dados de status corrente com erros de classificação.

Tais erros são devidos à sensibilidade e à especificidade do teste clínico utilizado para determinação da presença da doença de interesse no instante de observação.

O método estudado reduz substancialmente o vício quando comparado com o estimador não corrigido. O mesmo se observou para a versão suavizada do estimador corrigido via núcleo-estimador.

Palavras Chave: estimação não-paramétrica de máxima verossimilhança; dados de status corrente, erro de classificação. 


\section{Abstract}

We studied the performance of a method proposed to correct the nonparametric maximum likelihood estimator of the distribution function of failure time for current status data with misclassifications caused by sensitivity and specificity of the clinical test.

The corrected estimator reduced substantially the bias compared to the original estimator. The same was observed for the smoothed version of the corrected estimator.

key words: nonparametric maximum likelihood estimation; current status data; misclassification. 


\section{Introdução}

A análise de sobrevivência engloba metodologias de análise de dados em situações em que a variável resposta corresponde a um tempo até a ocorrência de algum evento de interesse. Pode-se ainda entender que o tempo de falha é o período de tempo que decorre para que o evento de interesse aconteça e a falha é exatamente a ocorrência do evento de interesse.

A principal característica na análise de sobrevivência é a presença de censuras.

Em estudos que envolvem dados de status corrente, temos censura à direita ou à esquerda da variável tempo de falha. Tais censuras são representadas por uma variável indicadora que pode ser registrada com erro. Esse erro pode ser devido à sensibilidade e à especificidade do teste utilizado para determinar seu valor.

A consequência desse erro é o incremento no vício do estimador não paramétrico de máxima verossimilhança (ENPMV) da distribuição do tempo de falha.

Neste estudo, abordamos uma metodologia para correção das estimativas não paramétricas da função de distribuição do tempo de falha, proposta por Gomes e daSilva (2011) e que fornece estimativas corrigidas semelhantes às obtidas utilizando-se o método de correção proposto por McKewon e Jewell (2010).

A metodologia proposta reduz substancialmente o vício do estimador não paramétrico de máxima verossimilhança (ENPMV).

Estudamos também uma versão suavizada via núcleo estimador (Kernel) do ENPMV, utilizando núcleo gaussiano. 


\section{Capítulo 1}

\section{Conceitos Básicos em Análise de Sobrevivência}

Os métodos de análise de sobrevivência são utilizados em estudos em que a variável de interesse representa o tempo até a ocorrência de um evento de interesse, a reincidência de uma doença ou a morte de um paciente, por exemplo. O evento de interesse pode estar associado ao resultado de testes, os quais podem apresentar erros de classificação. As medidas de sensibilidade e especificidade permitem incorporar a qualidade do teste na análise. Contudo, apesar de muitos modelos utilizados considerarem tais medidas, há alguns que não possuem informações sobre tais parâmetros, apresentando falta de identificabilidade.

O instante de ocorrência do evento de interesse é denominado "tempo de falha", podendo ser observado parcialmente (censurado). A censura pode ocorrer por inúmeras razões, tais como a saída do indivíduo do estudo por motivos não relacionados ao evento de interesse ou a não ocorrência do evento de interesse ao fim do experimento, entre outros.

\subsection{Conceitos}

\subsubsection{Censura}

A ocorrência de dados censurados é uma característica muito comum na Aná- 
lise de Sobrevivência. Esse tipo de dado fornece informações parciais sobre o valor da variável de interesse.

Mesmo censurados, esses dados devem ser incluídos na análise, pois apesar de estarem incompletos eles fornecem informações importantes e necessárias sobre o tempo de vida da variável de interesse em estudo e possíveis omissões podem provocar conclusões viciadas.

As censuras são especificadas de diferentes formas, podendo ocorrer pelos mais diversos motivos.

Censura do Tipo I: para um determinado experimento, o tempo de duração é pré-determinado e ao final deste tempo não ocorre falha para um ou mais elementos em estudo. A informação sobre o tempo de falha destes elementos será, portanto, incompleta.

Censura do Tipo II: o estudo é conduzido até que um número $\mathrm{k}(k \leq n)$ de elementos falhe. Ou seja, os indivíduos são acompanhados até obter-se um número pré-determinado de falhas.

Censura aleatória: o tempo de censura é aleatório quando os elementos observados falham por motivos distintos do interesse do estudo.

Censura Intervalar: somente é conhecido que o evento de interesse ocorreu em um certo intervalo de tempo. Em outras palavras, o tempo de falha não é conhecido exatamente, mas pertence a um intervalo. Ocorre, por exemplo, em estudos em que pacientes são acompanhados em visitas periódicas.

Dados agrupados de Sobrevivência: há casos de dados agrupados de sobrevivência, ou seja, quando há a ocorrência de censura intervalar e quando os instantes de tempo são os mesmos para os indivíduos avaliados.

\subsubsection{Função de distribuição acumulada e funções relaciona- das}

Seja T o tempo até a ocorrência de algum evento específico, genericamente chamado de falha. Tal evento pode ser definido como o aparecimento de um tumor, a morte de um paciente, o desenvolvimento de alguma doença, a recorrência de uma doença, a avaria de um equipamento, a cessação do aleitamento materno, a remissão 
após algum tratamento, a concepção, a cessação do tabagismo.

A variável aleatória positiva $T$ que representa o tempo de falha é normalmente caracterizada em análise de sobrevivência pela sua função de sobrevivência. Esse tempo de sobrevivência é caracterizado como sendo até a ocorrência do evento de interesse.

O tempo de sobrevivência (falha) está relacionado ao tempo de origem, o qual deve ser precisamente definido.

Para melhor compreensão, considere que para cada indivíduo $i, i=1,2, \ldots, n$, as observações serão representadas por $\left(t_{i}, \delta_{i}\right)$ em que $t_{i}$ é o tempo observado de falha ou de censura e $\delta_{i}$ é a variável que indica a ocorrência ou não de censura, dada por:

$$
\delta_{i}=\left\{\begin{array}{l}
0, \text { se } t_{i} \text { é tempo de censura } \\
1, \text { se } t_{i} \text { é tempo de falha. }
\end{array}\right.
$$

Pode-se ainda considerar no estudo covariáveis, $x_{i}$, (como por exemplo, sexo, idade, estado civil, tipo de tratamento) tendo-se então o vetor $\left(t_{i}, \delta_{i}, x_{i}\right)$ observado para o i-ésimo indivíduo.

Definição 1.1.1. A função de distribuição acumulada é dada por:

$$
F(t)=P(T \leq t)=\int_{0}^{t} f(u) d u
$$

onde $f($.$) é a função densidade de probabilidade de T$.

Considere ainda $S(t)$ a função de sobrevivência da variável aleatória não negativa T. Tal função definirá a probabilidade de um indivíduo sobreviver além de um certo tempo t. Ou seja, a quantidade básica empregada para descrever fenômenos de tempo para ocorrência do evento é:

Definição 1.1.2. a função de sobrevivência, que é denotada por:

$$
S(t)=P(T>t)=1-F(t)=\int_{t}^{\infty} f(u) d u
$$

sendo que quando $t=0, S(t)=1$ e quando $t \rightarrow \infty, S(t) \rightarrow 0$.

Como exemplo, considere uma função para a distribuição Weibull. A função de sobrevivência $S(t)=\exp \left(-\lambda t^{\alpha}\right), \quad \lambda>0, \alpha>0$. A distribuição exponencial é um 
caso especial da distribuição Weibull quando $\alpha=1$. A Curva de sobrevivência com a mediana comum de 6.93 foi exibida na Figura 1.1 para $\lambda=0.26328, \alpha=0.5 ; \lambda=0.1$, $\alpha=1 ;$ e $\lambda=0.00208, \alpha=3$

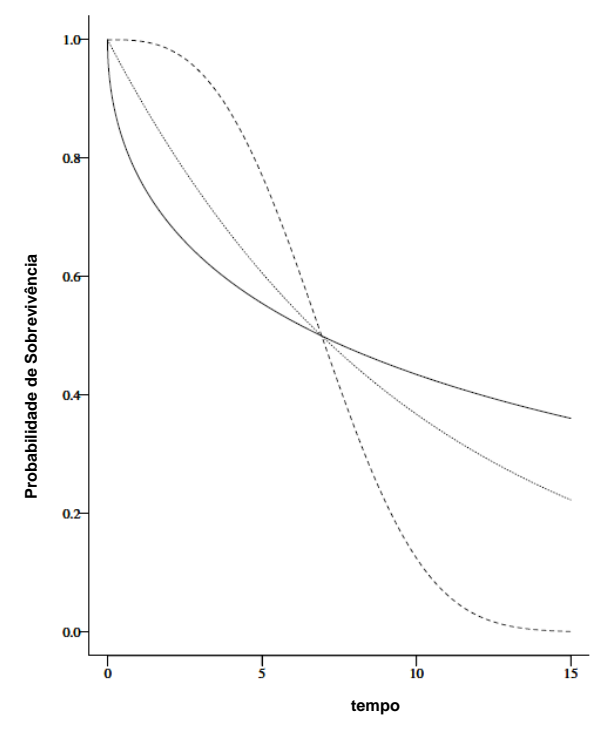

Figura 1.1: Função de sobrevivência da Weibull para $\alpha=0.5, \lambda=0.26328(\square)$; $\alpha=1.0, \lambda=0.1(\cdots \cdots) ; \alpha=3.0, \lambda=0.00208(-\cdots)$

Muitos tipos de curvas de sobrevivência podem ser mostradas, mas o ponto importante a ser destacado é que todas possuem as mesmas propriedades básicas. São monótonas e não crescente e, à medida que o tempo se aproxima de zero a função se aproxima de um e à medida que o tempo tende ao infinito, a função aproxima-se do zero. A taxa de declínio varia de acordo com o risco de ocorrência do evento no tempo t, mas é difícil determinar a essência de um padrão de falha, simplesmente observando a curva de sobrevivência. No entanto, esta quantidade continua a ser uma descrição popular de sobrevivência na literatura aplicada e pode ser muito útil na comparação de dois ou mais padrões de mortalidade.

Quando T é uma variável discreta, técnicas diferentes são necessárias para o estudo. A análise de sobrevivência para variáveis aleatórias discretas surge devido a erros de arredondamento, erros de falha nos intervalos ou quando o tempo de vida se refere a um número inteiro de unidades.

Definição 1.1.3. Suponha que T possa assumir valores $t_{j}, j=1,2, \ldots$ com função massa de probabilidade (f.m.p) $p\left(t_{j}\right)=\operatorname{Pr}\left(T=t_{j}\right), \quad j=1,2, \ldots$ quando $t_{1}<t_{2}<\ldots$ 
A função de sobrevivência para uma variável aleatória discreta T é dada por:

$$
S(t)=\operatorname{Pr}(T>t)=\sum_{t_{j}>t} p\left(t_{j}\right)
$$

Como exemplo, considerando o tempo de vida $\mathrm{T}$ com uma função de massa de probabilidade $p\left(t_{j}\right)=\operatorname{Pr}(T=j)=1 / 3, \quad j=1,2,3$ de uma distribuição uniforme discreta. A correspondente função de sobrevivência é identificada na expressão (1.4) e visualizada na Figura 1.2:

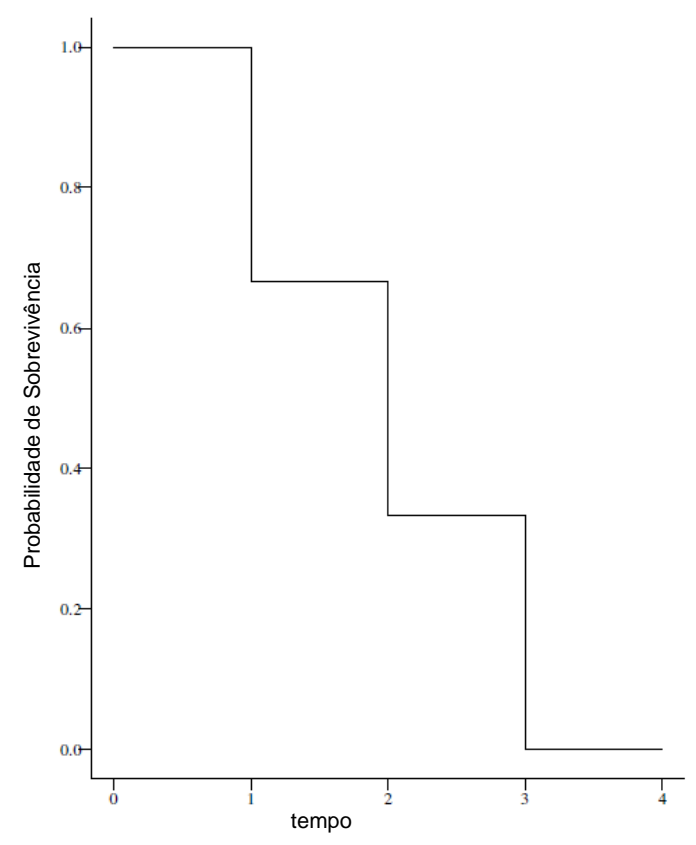

Figura 1.2: Função de Sobrevivência para o tempo de vida de uma variável aleatória discreta

$$
S(t)=\operatorname{Pr}(T>t)=\sum_{t_{j}>t}= \begin{cases}1 & , \text { se } 0 \leq t<1 \\ 2 / 3 & , \text { se } 1 \leq t<2 \\ 1 / 3 & , \text { se } 2 \leq t<3 \\ 0 \quad & , \text { se } t \geq 3\end{cases}
$$

Observe que, quando T é discreto, a função de sobrevivência é uma função escada decrescente.

A função taxa de falha, $h(t)$, é uma forma de quantificar o risco instantâneo de um acontecimento ocorrer por unidade de tempo, desempenhando um papel importante na análise de sobrevivência, Lee (1992). A função taxa de falha mostra a 
forma com que a taxa instantânea muda com o tempo, ou seja, como o risco de um indivíduo falhar no tempo $t+\Delta t$, com $\Delta t \rightarrow 0$, dado que ele sobreviveu ao tempo $t$. Essa taxa de falha é definida como:

$$
h(t)=\lim _{\Delta t \rightarrow 0} \frac{P(t \leq T<t+\Delta t \mid T \geq t)}{\Delta t}=\frac{f(t)}{S(t)}=-\frac{d}{d t} \log (S(t)) .
$$

A função taxa de falha acumulada $\mathrm{H}(\mathrm{t})$ é definida como:

$$
H(t)=\int_{0}^{t} h(u) d u
$$

As funções de sobrevivência e de probabilidade podem ser relacionadas através da expressão:

$$
S(t)=\exp \left(-\int_{0}^{t} h(u) d u\right)
$$

Pode-se ainda mostrar que:

$$
f(t)=h(t) \exp \left(-\int_{0}^{t} h(u) d u\right) .
$$

\subsection{Estimação da função de sobrevivência}

\subsubsection{Estimação paramétrica}

O método de máxima verossimilhança utilizado para estimar os parâmetros de um modelo estatístico, permite que se tenha uma opção apropriada para dados censurados, além dos estimadores possuírem propriedades favoráveis para grandes amostras.

Um bom exemplo a ser estudado seria a utilização da distribuição do tempo de ocorrência do evento como sendo uma distribuição Weibull. Portanto, através do estimador de máxima verossimilhança, obtêm-se os parâmetros que melhor se adequem à amostra observada.

Assim, se uma determinada população é caracterizada pela função de densidade $f(t ; \theta)$, onde $\theta$ é o vetor de parâmetros, a função de verossimilhança $L(\theta)$, é dada por:

$$
L(\theta)=\prod_{i=1}^{n} f\left(t_{i} ; \theta\right)
$$

para uma amostra sem dados censurados. 
Para dados com censura à direita, a contribuição de cada elemento da amostra para a construção da função de verossimilhança é dada por:

$$
\begin{cases}f\left(t_{i} \mid \theta\right) & \text {, se o } i \text {-ésimo tempo de sobrevivência foi observado, } \\ S\left(t_{i} \mid \theta\right) & \text {, se o } i \text {-ésimo tempo de sobrevivência foi censurado (censura à direita). }\end{cases}
$$

Então, pode-se dizer que a contribuição de cada observação não censurada na função de verossimilhança $L(\theta)$ é dada pela função de densidade.

As observações censuradas informam apenas que o tempo até a ocorrência do evento de interesse é maior que o tempo de censura observado.

A função de verossimilhança $L(\theta)$, então, é dada por:

$$
L(\theta)=\prod_{i=1}^{n}\left[f\left(t_{i} ; \theta\right)\right]^{\delta_{i}}\left[S\left(t_{i} ; \theta\right)\right]^{1-\delta_{i}}
$$

sendo, $\delta_{i}$ a variável indicadora de falha. Pode-se observar que quando $\delta_{i}=1, t_{i}$ será considerado como tempo de falha e quando $\delta_{i}=0, t_{i}$ será interpretado como tempo de censura.

Se considerada, por exemplo, a distribuição exponencial, sua função de verossimilhança será dada por:

$$
\begin{gathered}
L(\lambda)=\prod_{i=1}^{n}\left[\lambda \exp \left(-\lambda t_{i}\right)\right]^{\delta_{i}}\left[\exp \left(-\lambda t_{i}\right)\right]^{1-\delta_{i}} \\
L(\lambda)=\prod_{i=1}^{n} \lambda^{\delta_{i}}\left[\exp \left(-\lambda t_{i}\right)\right]=\lambda^{\sum_{i=1}^{n} \delta_{i}} \exp \left(-\lambda \sum_{i=1}^{n} t_{i}\right) .
\end{gathered}
$$

Seguindo o estudo de uma forma análoga, para a função Weibull sua função de verossimilhança será dada por:

$$
\begin{gathered}
L(\gamma, \lambda)=\prod_{i=1}^{n}\left[\frac{\gamma}{\lambda \gamma} t_{i}^{\gamma-1} \exp \left(-\left(\frac{t_{i}}{\lambda}\right)^{\gamma}\right)\right]^{\delta_{i}}\left[\exp \left(-\left(\frac{t_{i}}{\lambda}\right)^{\gamma}\right)\right]^{1-\delta_{i}} \\
L(\gamma, \lambda)=\prod_{i=1}^{n}\left[\frac{\gamma}{\lambda^{\gamma}} t_{i}^{\gamma-1}\right]^{\delta_{i}} \exp \left(-\left(\frac{t_{i}}{\lambda}\right)^{\gamma}\right) .
\end{gathered}
$$

O vetor de estimadores de verossimilhança que maximizam a função $L(\theta)$ será o mesmo que maximiza o logaritmo da função de verossimilhança $\log L(\theta)$. 
A resolução será a partir do seguinte sistema de equações:

$$
U\left(\theta_{j}\right)=\frac{\partial \log L(\theta)}{\partial\left(\theta_{j}\right)}, j=1, \ldots, k,
$$

onde k é o número de parâmetros.

Todavia, nesse sistema de equações, as equações são não lineares, não possuindo, portanto, solução analítica. A determinação da solução será dada numericamente, utilizando o método numérico de Newton-Raphson, por exemplo.

O modelo de regressão da distribuição Weibull é dado por:

$$
h\left(t \mid \boldsymbol{x}_{i}\right)=h_{0} \exp \left(\beta_{1} x_{i 1}+\beta_{2} x_{i 2}+\ldots+\beta_{p} x_{i p}\right)=h_{0}(t) \exp \left(\boldsymbol{\beta}^{\prime} \boldsymbol{x}_{i}\right)
$$

sendo que $h_{0}(t)=\frac{\gamma}{\lambda^{\gamma}} t^{\gamma-1}, \quad t \geq 0$ e, portanto,

$$
h\left(t \mid \boldsymbol{x}_{i}\right)=\frac{\gamma}{\lambda^{\gamma}} t^{\gamma-1} \exp \left\{\boldsymbol{\beta}^{\prime} \boldsymbol{x}_{i}\right\}
$$

Para esse modelo, os tempos de sobrevivência possuem uma distribuição de Weibull, implicando uma forma paramétrica particular sobre $h_{0}(t)$.

Com a forma da função de taxa de falha, pode-se observar que o tempo de sobrevivência do i-ésimo indivíduo possui uma distribuição de Weibull com parâmetro de escala

$$
\frac{\exp \left(\boldsymbol{\beta}^{\prime} \boldsymbol{x}_{i}\right)}{\lambda^{\gamma}}
$$

e parâmetro de forma $\gamma$.

Por esse resultado, pode-se perceber o efeito das covariáveis no modelo, alterando o parâmetro de escala da distribuição e mantendo o parâmetro de forma constante.

Pela função de taxa de falha, tem-se que

$$
\frac{h\left(t \mid \boldsymbol{x}_{i}\right)}{h_{0}\left(t \mid \boldsymbol{x}_{i}\right)}=k
$$

e

$$
h\left(t \mid \boldsymbol{x}_{i}\right)=k h_{0}\left(t \mid \boldsymbol{x}_{i}\right)=k \frac{\gamma}{\lambda^{\gamma}} t^{\gamma-1} .
$$

Fazendo $\frac{1}{\lambda}=\lambda^{*}$, tem-se que $h\left(t \mid \boldsymbol{x}_{i}\right)=k\left(\lambda^{*}\right)^{\gamma} \gamma t^{\gamma-1}$ e supondo que $\lambda^{*}=$ $\exp \left(\boldsymbol{\beta}^{\prime} \boldsymbol{x}_{i}\right)$, tem-se que

$$
h\left(t \mid \boldsymbol{x}_{i}\right)=k\left[\exp \left(\boldsymbol{\beta}^{\prime} \boldsymbol{x}_{i}\right)\right]^{\gamma} \gamma t^{\gamma-1}
$$




$$
\begin{gathered}
=k \exp \left(\gamma \boldsymbol{\beta}^{\prime} \boldsymbol{x}_{i}\right) \gamma t^{\gamma-1} \\
=\exp (\log k) \exp \left(\gamma \boldsymbol{\beta}^{\prime} \boldsymbol{x}_{i}\right) \gamma t^{\gamma-1} \\
=\exp \left[\log (k)+\gamma\left(\boldsymbol{\beta}^{\prime} \boldsymbol{x}_{i}\right)\right] \gamma t^{\gamma-1} \\
=\exp \left[\gamma \beta_{0}+\gamma\left(\boldsymbol{\beta}^{\prime} \boldsymbol{x}_{i}\right)\right] \gamma t^{\gamma-1} \\
=\exp \left(\boldsymbol{\beta}^{*^{\prime}} \boldsymbol{x}_{i}^{*}\right) \gamma t^{\gamma-1} .
\end{gathered}
$$

A função de sobrevivência correspondente é dada por:

$$
\begin{aligned}
S\left(t \mid \boldsymbol{x}_{i}\right) & =\exp \left\{-\int_{0}^{t} \exp \left(\boldsymbol{\beta}^{*^{\prime}} \boldsymbol{x}_{i}^{*}\right) \gamma u^{\gamma-1} d u\right\} \\
& =\exp \left\{-\exp \left(\boldsymbol{\beta}^{*^{\prime}} \boldsymbol{x}_{i}^{*}\right) t^{\gamma}\right\} .
\end{aligned}
$$

Por essa expressão, pode ser obtida a função de densidade de probabilidade, através de sua diferenciação em relação a $t$ e multiplicando-se o resultado obtido por $(-1)$ ou como resultado da relação:

$f(t)=h(t) S(t)$, ou seja,

$$
f\left(t \mid \boldsymbol{x}_{i}\right)=\exp \left(\beta^{*^{\prime}} \boldsymbol{x}_{i}^{*}\right) \gamma t^{\gamma-1} \exp \left\{-\exp \left({\beta^{*}}^{\prime} \boldsymbol{x}_{i}^{*}\right) t^{\gamma}\right\}
$$

Com a função de verossimilhança das observações e maximização dessa função em relação aos parâmetros desconhecidos, pode-se ajustar o modelo de riscos proporcionais de Weibull.

\subsubsection{Estimação não paramétrica da função de sobrevivência}

Na estimação da função de sobrevivência através de uma abordagem do tipo não paramétrica, não se faz nenhuma suposição sobre a forma matemática da distribuição do tempo de falha. Essa abordagem é muito útil para a escolha de um possível modelo teórico paramétrico.

O mais conhecido e utilizado estimador não paramétrico da função de sobrevivência é o estimador de Kaplan-Meier (1958), também chamado de estimador produto-limite. Este estimador é utilizado para estimação da função de sobrevivência quando observamos o tempo de falha ou temos censura à direita.

O estimador produto-limite é definido como: 


$$
\hat{S}(t)=\prod_{i \mid t_{i} \leq t}\left(1-\frac{d_{i}}{n_{i}}\right),
$$

onde $d_{i}$ e $n_{i}$ significam o número de eventos de interesse (falhas) ocorridos no tempo $t_{i}$ e o número de dados sob risco de ocorrência do evento (aqueles que não falharam ou que ainda não foram censurados até o tempo $t_{i}$ ), respectivamente. Considera-se ainda que $t_{1}<t_{2}<\ldots<t_{k}$ correspondem aos k tempos distintos (ordenados).

O estimador não paramétrico facilitará a identificação da função de sobrevivência. Isto poderá ser feito através de análises gráficas.

Pode-se utilizar, sob certas condições de regularidade, algumas propriedades assintóticas de $\hat{S}(t)$ (como por exemplo, normalidade e consistência) que permitem que se construam intervalos de confiança aproximados para $S(t)$ em um instante de tempo $t$.

Um intervalo aproximado de $95 \%$ de confiança para $S(t)$, é dado por:

$$
\hat{S}(t)^{\exp ( \pm 1.96 \sqrt{\operatorname{Var}(\hat{U}(t))})},
$$

onde a variância assintótica de $\hat{U}(t)$ :

$$
\hat{U}(t)=\log (-\log (\hat{S}(t)))
$$

é

$$
\operatorname{Var}(\hat{U}(t))=\frac{\sum_{i \mid t_{i} \leq t} \frac{d_{i}}{n_{i}\left(n_{i}-d_{i}\right)}}{\left[\sum_{i \mid t_{i} \leq t} \log \frac{\left(n_{i}-d_{i}\right)}{n_{i}}\right]^{2}}
$$

que assume valores no intervalo de $[0,1]$.

Há ainda outros estimadores para a função de sobrevivência, como o estimador da tabela de vida e o estimador de Nelson-Aalen (vide Colosimo, (2001), para mais informações).

\subsection{Modelo de regressão de Cox}

A utilização de covariáveis relacionadas com o tempo de sobrevida está frequentemente presente em estudos de análise de sobrevivência. A verificação dos efeitos dessas covariáveis nos tempos de sobrevivência pode ser feita através do modelo de risco proporcional. 
A função de risco no tempo t condicionada a um vetor de covariáveis $\boldsymbol{x}$ pode ser expressa como:

$$
h(t \mid \boldsymbol{x})=h_{0}(t) g(\boldsymbol{\beta}, \boldsymbol{x}),
$$

onde g representa uma função não negativa que deverá ser especificada, $\boldsymbol{x}$ representa o vetor de covariáveis e $\boldsymbol{\beta}$ representa o vetor de parâmetros relacionado às covariáveis.

Esse modelo é resultado da composição de dois componentes, um não paramétrico e outro paramétrico, sendo portanto um modelo do tipo semiparamétrico. $h_{0}(t)$ será o componente não paramétrico (não especificado) sendo portanto uma função positiva no tempo.

O componente $g(\boldsymbol{\beta}, \boldsymbol{x})$ pode ser expressa como:

$$
g(\boldsymbol{\beta}, \boldsymbol{x})=\exp \left(\boldsymbol{\beta}^{\prime} \boldsymbol{x}\right)=\exp \left(\beta_{1} x_{1}+\beta_{2} x_{2}+\ldots+\beta_{p} x_{p}\right) .
$$

No caso de $\boldsymbol{x}=0$, temos $h(t)=h_{0}(t)$.

Vale ressaltar que o componente não paramétrico no modelo absorve a constante $\boldsymbol{\beta}_{\mathbf{0}}$ encontrada nos modelos paramétricos.

O modelo acima descrito pode também ser escrito como:

$$
h(t \mid \boldsymbol{x})=h_{0}(t) \exp \left(\beta_{1} x_{1}+\beta_{2} x_{2}+\ldots+\beta_{p} x_{p}\right) .
$$

Portanto, nesse modelo, o vetor de covariávies $\boldsymbol{x}$ será dado por $\boldsymbol{x}=\left(x_{1}, x_{2}, \ldots, x_{p}\right)$. O tempo de sobrevivência será $t_{i}$ para o i-ésimo indivíduo dependente dessas covariáveis. A expressão (1.20) é denominada de risco proporcional de Cox.

A razão das taxas de risco de indivíduos diferentes não depende do tempo.

$$
\frac{h_{i}(t)}{h_{j}(t)}=\frac{h_{0}(t) \exp \left(\beta_{i} x_{i}\right)}{h_{0}(t) \exp \left(\beta_{j} x_{j}\right)}=\exp \left(\beta_{i} x_{i}-\beta_{j} x_{j}\right)
$$




\section{Capítulo 2}

\section{Dados de status corrente com erro de classificação}

Dados de status corrente ocorrem em muitos campos, incluindo experimentos cancerígenos em animais, estudos demográficos, econométricos, epidemiológicos, estudos de reabilitação. Em muitas situações, tais como experimentos cancerígenos em tumores ocultos, dados de status corrente são as únicas informações disponíveis a respeito de variáveis de sobrevivência de interesse tais como o tempo de acometimento de um tumor. Por isso, as variáveis de sobrevivência não podem ser diretamente mensuradas. Um exemplo de tal situação são estudos epidemiológicos onde o evento de interesse é o acometimento de uma certa doença crônica. Outro exemplo é dado pelo estudo demográfico onde o evento de interesse pode ser a primeira gravidez ou casamento.

Nos dados de status corrente o valor do tempo da variável T para ocorrência do evento é conhecida somente como sendo maior ou menor que o valor da variável observada C.

Alguns métodos para deteç̧ão de um evento podem estar ligados a exames clínicos, os quais sujeitam-se a erros de classificação. Ao estimar a função de distribuição $\mathrm{F}$ do tempo de falha, precisa-se considerar o efeito da sensibilidade e especificidade do teste de diagnóstico. 


\subsection{Conceitos de sensibilidade e especificidade}

Nos casos em que o evento de interesse é a ocorrência de uma doença, os erros mais comuns cometidos são a classificação de um indivíduo doente como sadio ou a classificação de um indivíduo sadio como doente. A quantificação da qualidade dos testes é feita através das medidas de sensibilidade e especificidade. Sensibilidade é a probabilidade do teste estudado classificar corretamente um indivíduo doente. A especificidade é definida como a probabilidade de classificar corretamente os indivíduos que não possuem a doença.

Seja " $s$ " a sensibilidade de um teste, " $e$ " a especificidade de um teste e $\gamma$ o indicador do resultado do teste, conforme definido a seguir:

$$
\delta=\left\{\begin{array}{l}
1, \text { se o indivíduo está de fato doente } \\
0, \text { se o indivíduo está de fato sadio }
\end{array}\right.
$$

$\gamma= \begin{cases}1 & , \text { resultado do teste indica que o indivíduo está doente } \\ 0 & , \text { resultado do teste indica que o indivíduo está sadio. }\end{cases}$

A sensibilidade e a especificidade, respectivamente, são dadas por

$$
s=P(\gamma=1 \mid \delta=1)
$$

e

$$
e=P(\gamma=0 \mid \delta=0)
$$

Observe que um teste seria, então, considerado bom quando possui altos valores para a sensibilidade e também para a especificidade, de modo a ter poucos falsos positivos e poucos falsos negativos.

Se os testes de diagnósticos forem perfeitos (ou seja, para sensibilidade e especificidade iguais a 1), podem-se utilizar os métodos tradicionais de análise de sobrevivência. Contudo, se existem erros ou se estes erros são ignorados, o processo de inferência sobre os parâmetros da distribuição dos tempos de falha pode ser seriamente comprometido. 


\subsection{Estimador não-paramétrico de máxima verossi- milhança}

Para dados de status corrente, o estimador não paramétrico de máxima verossimilhança (ENPMV) da função de distribuição é obtido utilizando-se a técnica de regressão isotônica.

Precisamos ter uma visão geral da teoria de regressão isotônica, com o objetivo de obter conhecimentos necessários para entender suas propriedades.

Consideremos uma dada função arbitrária g, definida em algum espaço X. A regressão isotônica $g^{*}$ é a solução do problema de mínimos quadrados ponderados:

$$
\min _{f \in \Im} \sum_{x \in X}[g(x)-f(x)]^{2} w(x),
$$

sendo $\Im$ o conjunto de funções não decrescentes definidas em X.

\subsubsection{Otimização Convexa}

Para manter a simplicidade, podemos nos restringir à situação, onde $S \subset \mathbb{R}^{k}$ é convexo e $\phi$ é a função convexa a ser minimizada. O objetivo é estabelecer condições necessárias e suficientes para otimização, que permitem uma interpretação geométrica em termos da função de minorante convexo sobre o $\mathbb{R}$.

Definição 2.2.1. Um conjunto $S \subset \mathbb{R}^{k}$ é convexo se para cada par de pontos $p, q \in S$, o ponto $r$ no segmento de reta que une os pontos $p$ e q estará também no conjunto $S$.

Definição 2.2.2. Uma função real $\phi$ é chamada convexa, se para cada dois pontos $x$ e y no domínio e algum $t \in[0,1]$, teremos

$$
\phi(t x+(1-t) y) \leq t \phi(x)+(1-t) \phi(y)
$$

No Lema seguinte, através da interpretação geométrica da função convexa, relacionamos as condições necessárias e suficientes para um ponto $s \in S$ ser o ideal.

Lema 2.2.1. (Jongbloed, 1999) Seja ф uma função convexa continuamente diferenciável no conjunto convexo $S \subset \mathbb{R}^{k}$. Denote por $\nabla \phi$ o vetor de derivadas parciais de $\phi$ com respeito aos componentes de s. Então,

$$
\hat{s} \in \arg \min _{s \in S} \phi(s)
$$


se e somente se

$$
\hat{s} \in S \quad \text { e para } \forall s \in S, \quad \nabla \phi(\hat{s})^{T}(s-\hat{s}) \geq 0 .
$$

Definição 2.2.3. Um conjunto $S \subset \mathbb{R}^{k}$ é um cone convexo, se além dos requisitos estabelecidos na Definição 2.2.1, S tem a propriedade que se $p \in S$ e $t$ é um número real não negativo então tp $\in S$.

Corolário 2.2.1. (Jongbloed, 1999) Suponha que $S$ é um cone convexo em $\mathbb{R}^{k}$ e que $\phi$ é convexo e continuamente diferenciável em S. Então,

$$
\hat{s} \in \arg \min _{s \in S} \phi(s)
$$

se e somente se

$$
\hat{s} \in S, \nabla \phi(\hat{s})^{T} \hat{s}=0 \quad \text { e } \forall s \in S, \quad \nabla \phi(\hat{s})^{T} s \geq 0 .
$$

Exemplo: Consideramos o problema de encontrar a altura média de 42 meninas de uma escola, onde as faixas etárias são de 12 a 18 anos. A monotonicidade da solução é importante para este exemplo. Obviamente, estes comprimentos médios devem aumentar em função de idade. Procedendo da seguinte forma: para cada idade foram medidas as alturas de cada grupo de seis garotas, veja Tabela 2.1 (Jongbloed, 1999), e assume-se ser gerado de forma independente pela distribuição normal, todos com a mesma variância, mas médias diferentes.

Tabela 2.1: Comprimento de 42 garotas de uma escola em diferentes idades

\begin{tabular}{|c|c|c|c|c|c|c|c|c|}
\hline idade $\mathbf{i}$ & & & & & & & $\overline{\boldsymbol{x}}(\mathbf{i}) \mathbf{- 1 . 6 0}$ & st-dev(i) \\
\hline 12 & 1.62 & 1.51 & 1.55 & 1.54 & 1.71 & 1.63 & -0.01 & 0.07 \\
\hline 13 & 1.67 & 1.61 & 1.83 & 1.54 & 1.53 & 1.55 & 0.02 & 0.11 \\
\hline 14 & 1.61 & 1.60 & 1.56 & 1.57 & 1.68 & 1.51 & -0.01 & 0.06 \\
\hline 15 & 1.70 & 1.56 & 1.66 & 1.68 & 1.75 & 1.50 & 0.04 & 0.09 \\
\hline 16 & 1.58 & 1.75 & 1.56 & 1.65 & 1.67 & 1.67 & 0.05 & 0.06 \\
\hline 17 & 1.84 & 1.66 & 1.71 & 1.82 & 1.79 & 1.88 & 0.18 & 0.08 \\
\hline 18 & 1.68 & 1.64 & 1.65 & 1.73 & 1.65 & 1.83 & 0.10 & 0.07 \\
\hline
\end{tabular}

Definimos que $\mu_{i-11}$ é o tamanho esperado de uma garota de idade i (então, para simplificar, temos os parâmetros $\left.\mu_{1}, \ldots, \mu_{7}\right)$. Se não considerarmos o conhecimento a priori de monotonicidade, nós estimaremos $\bar{x}(i)$ para diferentes tamanhos médios, utilizando os estimadores de máxima verossimilhança. De fato, escrevendo menos o 
logaritmo da verossimilhança em termos do vetor de parâmetros $\mu$, obteremos (ignorando os termos não dependentes de $\mu$ ):

$$
\phi(\mu)=\sum_{i=1}^{7}\left(\bar{x}_{i}-\mu_{i}\right)^{2}
$$

que é minimizado sobre o $\mathbb{R}^{7}$ e tomando $\hat{\mu}_{i}=\bar{x}_{i}$. Observando a Tabela 2.1, vemos que essas estimativas $\hat{\mu}_{i}$ não crescem como uma função da idade. Com o objetivo de encontrar uma solução, que aumente em função da idade, consideramos a "restrição de monotonicidade" (com as idades ordenadas). Portanto, a estimativa de máxima verossimilhança é obtida pela minimização de $\phi$ não sobre todo o $\mathbb{R}^{7}$ mas sobre o conjunto

$$
\left\{\mu \in \mathbb{R}^{7}: \mu_{1} \leq \mu_{2} \leq \ldots \leq \mu_{7}\right\}
$$

Antes de calcular esta estimativa, deve-se aprofundar as discussões sobre regressão isotônica.

Definição 2.2.4. A relação binária $(\prec)$ entre os elementos de $X$ é uma relação de ordem simples se (Barlow et al., 1972):

1) é reflexiva: $x \prec x$ para todo $x \in X$;

2) é transitiva: $x, y, z \in X, x \prec y$ e $y \prec z$ então $x \prec z$;

3) é anti-simétrica: $x, y \in X, x \prec y$ e $y \prec x$ então $x=y$ e

4) todo par de elementos de $X$ é comparável: $x, y \in X$, implica que, $x \prec y$ ou $y$ $\prec x$.

Definição 2.2.5. Seja $X$ um conjunto finito $\left\{x_{1}, x_{2}, \ldots, x_{k}\right\}$ com uma ordenação simples $x_{1} \prec x_{2} \prec \ldots \prec x_{k}$. Uma função $f$ em $X$ é isotônica (com respeito a essa ordenação) se $x, y \in X$ e $x \prec y$ implica $f(x) \leq f(y)$ e antitônica (com respeito a essa ordenação) se $x \prec y$ implica $f(x) \geq f(y)$.

Definição 2.2.6. Suponha $\mathbf{g}$ uma função em $X$ e $\omega$ uma dada função positiva em $X$. Uma função $g^{*}$ em $X$ é uma regressão isotônica de $g$ com pesos $\omega$ com respeito a uma ordenação simples $x_{1} \prec x_{2} \prec \ldots \prec x_{k}$ se essa função $g^{*}$ minimiza

$$
\sum_{x \in X}[g(x)-f(x)]^{2} \omega(x)
$$

para $f$ variando no conjunto de todas as funções isotônicas em X. 
Desta forma, $g^{*}$ é uma solução de mínimos quadrados restritos para a expressão acima.

Teorema 2.2.1. (Barlow et al., 1972) Uma regressão isotônica $g^{*}$ de g com pesos w

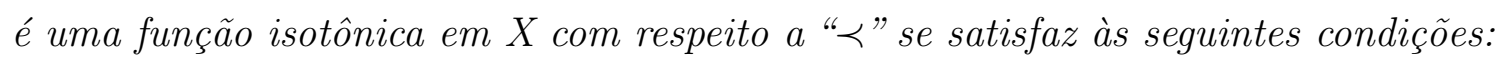

$$
\sum_{x \in X}\left[g(x)-g^{*}(x)\right]\left[g^{*}(x)-f(x)\right] w(x) \geq 0
$$

e

$$
\sum_{x \in X}[g(x)-f(x)]^{2} w(x) \geq \sum_{x \in X}\left[g(x)-g^{*}(x)\right]^{2} w(x)+\sum_{x \in X}\left[g^{*}(x)-f(x)\right]^{2} w(x)
$$

para cada função f em X. Consequentemente se uma função isotônica u satisfaz à condição

$$
\sum_{x \in X}[g(x)-u(x)][u(x)-f(x)] w(x) \geq 0 .
$$

para cada função isotônica f em X, haverá uma função u como uma regressão isotônica de g com pesos w. Existirá no máximo uma função isotônica.

Observação 2.2.1. Note que a propriedade especial da classe das funções isotônicas em $X$ usada na prova do teorema é o fato de ser convexo, ou seja, se $f_{1}$ e $f_{2}$ são isotônicas em $X$ e se $0 \leq \alpha \leq 1$ então $\alpha f_{1}+(1-\alpha) f_{2}$ será isotônica.

Prova: Primeiro nós mostramos que se $g^{*}$ é uma regressão isotônica de g, isto é, se $g^{*}$ é isotônica e minimiza

$$
\sum_{x}[g(x)-f(x)]^{2} w(x)
$$

na classe das funções f em X, então (2.6) é satisfeito. Se $0 \leq \alpha \leq 1$ e se f é isotônica, então pela observação $(2.2 .1),(1-\alpha) g^{*}+\alpha f$ será também isotônica. Por conseguinte,

$$
\sum_{x}\left\{g(x)-\left[(1-\alpha) g^{*}(x)+\alpha f(x)\right]\right\}^{2} w(x)
$$

assume seu mínimo em $\alpha=0$, o valor de $\alpha$ para cada $(1-\alpha) g^{*}(x)+\alpha f(x)=g^{*}$. Essa soma é uma função quadrática de $\alpha$, cuja derivada no ponto $\alpha=0$ será não negativa. A derivada com respeito a $\alpha$, no ponto $\alpha=0$ é justamente

$$
2 \sum_{x}\left[g(x)-g^{*}(x)\right]\left[g^{*}(x)-f(x)\right] w(x)
$$


e a equação (2.6) deve garantir por consequência que $g^{*}$ é a regressão isotônica de g. A inequação (2.7) segue da identidade:

$$
\begin{gathered}
{[g(x)-f(x)]^{2} w(x)=\left[g(x)-g^{*}(x)\right]^{2} w(x)+\left[g^{*}(x)-f(x)\right]^{2} w(x)} \\
+2\left[g(x)-g^{*}(x)\right]\left[g^{*}(x)-f(x)\right] w(x)
\end{gathered}
$$

observando que o último termo é positivo, fato decorrente de (2.6).

Suponha agora que u é uma função isotônica de X satisfazendo (2.8). Então, como descrito

$$
\sum_{x}[g(x)-f(x)]^{2} w(x) \geq \sum_{x}[g(x)-u(x)]^{2} w(x)+\sum_{x}[u(x)-f(x)]^{2} w(x)
$$

para toda função isotônica f. O último termo será não negativo e igual a 0 , se $f=u$ e u minimiza

$$
\sum_{x}[g(x)-f(x)]^{2} w(x)
$$

na classe de funções isotônicas de f; isto é, u é uma regressão isotônica de g. Completase a prova do teorema, mostrando que se duas funções isotônicas $u_{1}$ e $u_{2}$ são ambas satisfeitas, isto é, se

$$
\sum_{x}\left[g(x)-u_{1}(x)\right]\left[u_{1}(x)-f(x)\right] w(x) \geq 0
$$

$\mathrm{e}$

$$
\sum_{x}\left[g(x)-u_{2}(x)\right]\left[u_{2}(x)-f(x)\right] w(x) \geq 0
$$

para toda função isotônica $\mathrm{f}$, então $u_{1}=u_{2}$. Para isso, substitui-se $u_{2}$ para f na primeira inequação e $u_{1}$ para f na segunda inequação. Simplificadamente, teremos:

$$
-\sum_{x}\left[u_{1}(x)-u_{2}(x)\right]^{2} w(x) \geq 0
$$

Como cada termo na soma é obviamente não-negativo, cada termo deverá ser nulo. Mas $w(x)>0$ para cada $\mathrm{x}$, então $u_{1}(x)=u_{2}(x)$ para cada $\mathrm{x}$, isto é $u_{1}=u_{2}$.

Observação 2.2.2. É interessante notar que a inequação (2.6) segue diretamente da equação (2.3). Logo, se definir para o Lema (2.2.1) que $s=f(x), \hat{s}=g^{*}(x) e$

$$
\phi(s)=\phi(f(x))=\sum_{x \in X}[g(x)-f(x)]^{2} w(x)
$$


Então (2.3) implica que:

$$
2 \sum_{x \in X}(-1)\left[g(x)-g^{*}(x)\right]\left[f(x)-g^{*}(x)\right] w(x) \geq 0
$$

Teorema 2.2.2. (Barlow et al., 1972) Uma função isotônica $g^{*}$ em X é a regressão isotônica de g com pesos $w$ se e somente se

$$
\sum_{x \in X}\left[g(x)-g^{*}(x)\right] g^{*}(x) w(x)=0
$$

$e$

$$
\sum_{x \in X}\left[g(x)-g^{*}(x)\right] f(x) w(x) \leq 0,
$$

Para toda função isotônica f. A regressão isotônica $g^{*}$ satisfaz também

$$
\sum_{x \in X} g(x) w(x)=\sum_{x \in X} g^{*}(x) w(x)
$$

Pelo Teorema 2.2.1, segue a prova que será em seguida relacionada com a otimização convexa.

Observação 2.2.3. As propriedades (2.9) e (2.10) da regressão isotônica exigem uma propriedade adicional da classe de funções isotônicas, que são cônicas, isto é, se fé isotônica e c é uma constante não-negativa então cf será também isotônica.

Observação 2.2.4. A equação (2.9) e a inequação (2.10) seguem diretamente do corolário 2.2.1. Portanto, se definirmos $f(x), g^{*}(x)$ e $\phi(f(x))$ na observação 2.2.2, então a equação(2.3) implicará que

$$
2 \sum_{x \in X}(-1)\left[g(x)-g^{*}(x)\right] g^{*}(x) w(x)=0
$$

$e$

$$
2 \sum_{x \in X}(-1)\left[g(x)-g^{*}(x)\right] f^{*}(x) w(x) \geq 0 .
$$

As propriedades (2.9) e (2.10) são condições necessárias e suficientes para $g^{*}$ ser o ideal e ter uma boa interpretação geométrica no plano $\mathbb{R}^{2}$, a qual dará a possibilidade de estabelecer o modelo gráfico para o cálculo da regressão isotônica. 


\subsubsection{Interpretação Gráfica: função minorante convexa má- xima}

Assumimos uma ordenação simples $(\prec)$ em um conjunto finito $X=\left\{x_{1}, x_{2}, \ldots, x_{k}\right\}$.

A Figura 2.1 mostra um gráfico da soma acumulada ponderada

$$
G_{j}=\sum_{i=1}^{j} g\left(x_{i}\right) w\left(x_{i}\right), \quad j=1,2, \ldots, k
$$

e a soma acumulada

$$
W_{j}=\sum_{i=1}^{j} w\left(x_{i}\right), \quad j=1,2, \ldots, k .
$$

Isto é, plotamos os pontos $P_{j}=\left(W_{j}, G_{j}\right)$, para $j=1,2, \ldots, k$ e $P_{0}=(0,0)$, no plano Cartesiano. Esses pontos constituem o diagrama de somas acumuladas (DSA) da função g com pesos w. A inclinação do segmento que une os pontos $P_{j-1}$ a $P_{j}$ é dada por

$$
\frac{G_{j}-G_{j-1}}{W_{j}-W_{j-1}}=g\left(x_{j}\right)
$$

para $j=1,2, \ldots, k$. A inclinação do segmento de reta $P_{i-1}$ a $P_{j}$ para $i \leq j$ representa a média ponderada

$$
A v\left\{x_{i}, x_{i+1}, \ldots, x_{j}\right\}=\frac{\sum_{r=i}^{j} g\left(x_{r}\right) w\left(x_{r}\right)}{\sum_{r=i}^{j} w\left(x_{r}\right)} .
$$

Considerando $G^{*}$ como sendo a função minorante convexa máxima (MCM) do DSA no intervalo $\left[0, W_{k}\right]$ e o MCM é o gráfico do supremo de todas as funções convexas que estão abaixo do DSA. A função definida dessa forma é convexa em $\left[0, W_{k}\right]$. O gráfico do DSA e da MCM para um dado conjunto de pontos é dado na Figura 2.1 (Robertson et al., 1988).

Pelo fato da convexidade de $G^{*}$ estar em $\left[0, W_{k}\right], G^{*}$ deverá ser diferenciável em cada um dos pontos $W_{1}, W_{2}, \ldots, W_{k}$. Representam-se os pontos da MCM por $P_{j}^{*}=\left(W_{j}, G_{j}^{*}\right) \mathrm{com}$

$$
G_{j}^{*}=\sum_{i=1}^{j} g^{*}\left(x_{i}\right) w\left(x_{i}\right) .
$$

Portanto, o valor da regressão isotônica $g^{*}$ no ponto $x_{j}$ é somente a inclinação da MCM no ponto $P_{j}^{*}=\left(W_{j}, G_{j}^{*}\right)$.

Se $P_{j}^{*}$ for um ponto não diferenciável do gráfico, $g^{*}\left(x_{j}\right)$ será a inclinação do seguimento prolongado à esquerda. Se para algum i a MCM em $W_{i}$ for posta 


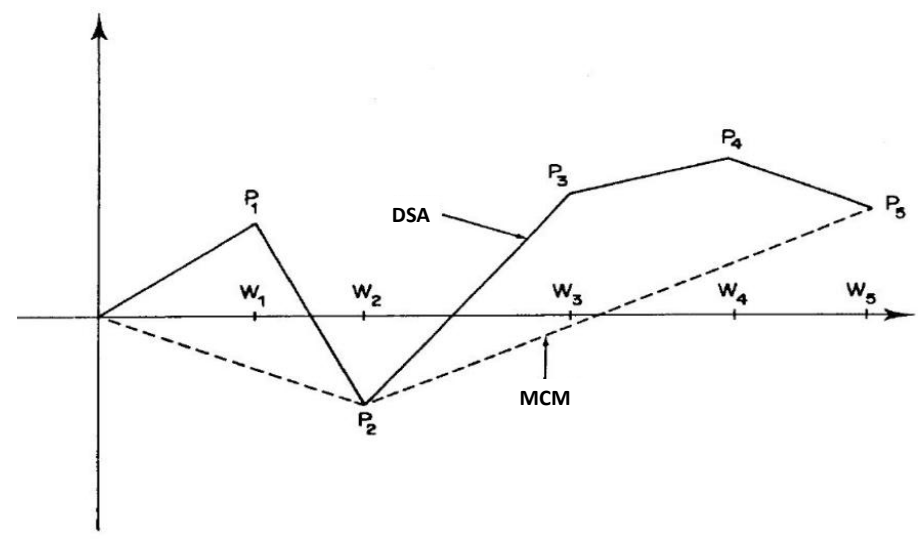

Figura 2.1: Diagrama das Somas Acumuladas (DSA) e a função Minorante Convexa Máxima (MCM)

estritamente abaixo do DSA, então a inclinação da MCM à esquerda e à direita de $W_{i}$ são os mesmos. Em outras palavras, para $i=1,2, \ldots, k-1$

$$
G^{*}\left(W_{i}\right)<G_{i} \Rightarrow g^{*}\left(x_{i+1}\right)=g^{*}\left(x_{i}\right)
$$

Teorema 2.2.3. (Robertson et al., 1988) Se X é uma ordenação simples, a derivada à esquerda ou a inclinação à esquerda, $g^{*}$, da MCM fornece a regressão isotônica de g. De fato, se fé isotônica em X, então:

$$
\sum_{x \in X}[g(x)-f(x)]^{2} w(x) \geq \sum_{x \in X}\left[g(x)-g^{*}(x)\right]^{2} w(x)+\sum_{x \in X}\left[g^{*}(x)-f(x)\right]^{2} w(x) .
$$

A regressão isotônica é única.

Exemplo (continuação): Para encontrar o mínimo de $\phi$, no exemplo dado anteriormente, sobre o cone $\left\{\mu \in \mathbb{R}^{7}: \mu_{1} \leq \mu_{2} \leq \ldots \leq \mu_{7}\right\}$, construímos os pontos $P_{i}$. Observando que os pesos são todos iguais a um, vemos que

$$
P_{i}=\left(i, \sum_{j=1}^{i}\left(\bar{x}_{j}-1.60\right)\right) \quad i=1, \ldots, 7
$$

Inicia-se o DSA pela origem. Veja a Figura 2.2 (Jongbloed, 1999) para os pontos, onde o eixo y é deslocado 1.60 para melhor visualização, e o seu minorante convexo máximo

$$
\hat{\mu}=(1.59,1.61,1.61,1.64,1.65,1.74,1.74)^{T},
$$




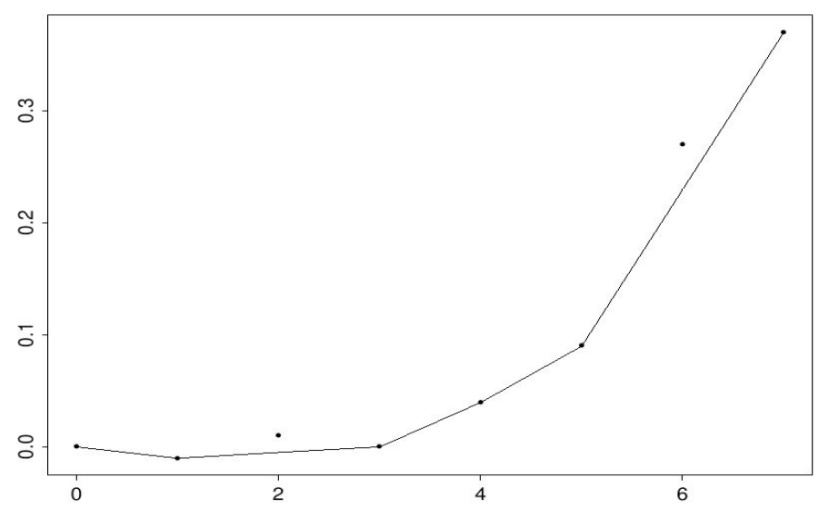

Figura 2.2: Diagrama das Somas Acumuladas e a Minorante Convexa Máxima para os dados da Tabela 2.1

que aumenta em função da idade.

Observação 2.2.5. Se g é antitônica, um mesmo argumento pode ser feito utilizando o Minimo Côncavo Majoritário em vez de MCM.

\subsubsection{A fórmula max-min}

Outra expressão que está intimamente relacionada à representação gráfica anterior (Figura 2.2) da regressão isotônica como a inclinação da MCM é a fórmula max-min.

Teorema 2.2.4. A regressão isotônica $g^{*}$ pode ser expressa como

$$
g^{*}\left(x_{i}\right)=\max _{s \leq i} \min _{t \geq i} A v\left(\left\{x_{s}, \ldots, x_{t}\right\}\right)
$$

onde

$$
A v\left(\left\{x_{s}, \ldots, x_{t}\right\}\right)=\frac{\sum_{j=s}^{t} g\left(x_{j}\right) w\left(x_{j}\right)}{\sum_{j=s}^{t} w\left(x_{j}\right)} .
$$

Fórmulas equivalentes são:

$$
\begin{aligned}
& g^{*}\left(x_{i}\right)=\min _{t \geq i} \max _{s \leq i} A v\left(\left\{x_{s}, \ldots, x_{t}\right\}\right) \\
& g^{*}\left(x_{i}\right)=\max _{s \leq i} \min _{t \geq i} A v\left(\left\{x_{s}, \ldots, x_{t}\right\}\right) \\
& g^{*}\left(x_{i}\right)=\min _{t \geq i} \max _{s \leq t} A v\left(\left\{x_{s}, \ldots, x_{t}\right\}\right) .
\end{aligned}
$$


Note que, se considerarmos uma função antitônica, o máximo e o mínimo da fórmula do teorema 2.2.4 deverão ser invertidos.

Na prática, as fórmulas max-min não são utilizadas. O algoritmo utilizado para a computação da regressão isotônica para uma ordenação simples é o PoolAdjacent-Violators Algorithm (PAVA).

\subsubsection{O Algoritmo Pool-Adjacent-Violators(PAV)}

Considere o gráfico dado pela Figura 2.3 (Robertson et al., 1988). Se a inclinação do segmento que une os pontos $P_{0}$ e $P_{1}$ ( $P_{0}$ é a origem do plano) é maior que a inclinação do segmento de reta que une os pontos $P_{1}$ e $P_{2}$ isso constituirá uma violação. Portanto, esses dois segmentos de retas são substituídos por um segmento de reta que une os pontos $P_{0}$ e $P_{2}$. Agora, se a inclinação do segmento de reta que une os pontos $P_{0}$ e $P_{2}$ for maior que a inclinação do segmento de reta que une os pontos $P_{2}$ e $P_{3}$, então esses dois segmentos serão substituídos por um segmento de reta que une os pontos $P_{0}$ e $P_{3}$. Então, para esse conjunto de dados, a MCM é determinada e consequentemente é a regressão isotônica.

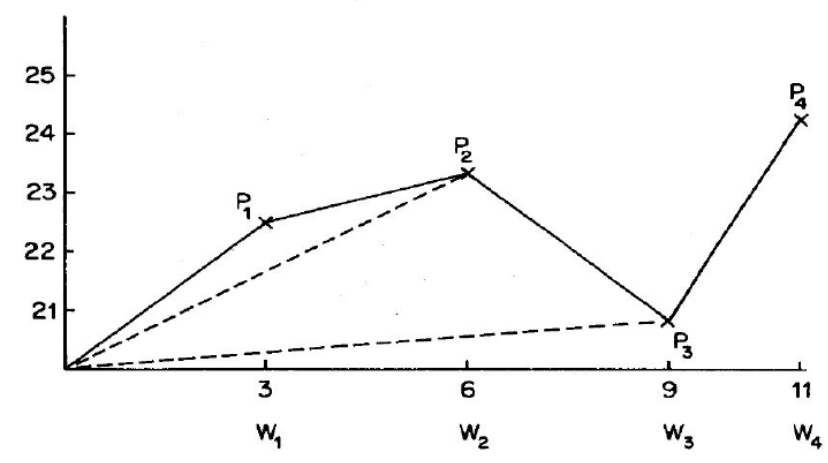

Figura 2.3: Interpretação gráfica do pooling-adjacent-violators

A regressão isotônica $g^{*}$ em subconjuntos de X de mesmo tamanho, isto é, conjuntos níveis para $g^{*}$, são chamados de soluções em blocos. Em cada um desses blocos de soluções, o valor de $g^{*}$ é a média ponderada dos valores de g sobre o bloco, usando o peso $w$; isto é, a inclinação do conjunto da MCM correspondente ao bloco. 
Assim, basta encontrar a solução dos blocos, isto é, conjuntos de elementos consecutivos de X em cada $g^{*}$ que assumem um valor particular. Na descrição do algoritmo, um conjunto arbitrário de elementos consecutivos de X estará referido a um bloco. O algoritmo inicia com a melhor partição possível dos blocos, nominalmente os pontos individuais de X, e une os blocos passo a passo até a partição final a ser alcançada.

$\mathrm{O}$ algoritmo PAV começa com g e procede conforme dado à seguir:

1) Se g é isotônica $\left(g\left(x_{1}\right) \leq g\left(x_{2}\right) \leq \ldots \leq g\left(x_{k}\right)\right)$, então sua partição inicial é também a partição final e $g^{*}\left(x_{i}\right)=g\left(x_{i}\right), \quad i=1,2, \ldots, k$ :

2) Por outro lado, se existe um violador i tal que $g\left(x_{i-1}\right)>g\left(x_{i}\right)$, esses dois valores são substituídos por suas médias ponderadas, nominalmente

$$
A v\left(\left\{x_{i-1}, x_{i}\right\}\right)=\frac{g\left(x_{i-1}\right) w\left(x_{i-1}\right)+g\left(x_{i}\right) w\left(x_{i}\right)}{w\left(x_{i-1}\right)+w\left(x_{i}\right)}
$$

e os dois pesos $w\left(x_{i-1}\right)$ e $w\left(x_{i}\right)$ são substituídos por $w\left(x_{i-1}\right)+w\left(x_{i}\right)$.

3) Se esse novo conjunto de $k-1$ valores é isotônico, isto é,

$$
g\left(x_{1}\right) \leq \ldots \leq g\left(x_{i-2}\right) \leq A v\left(\left\{x_{i-1}, x_{i}\right\}\right) \leq g\left(x_{i+1}\right) \leq \ldots \leq g\left(x_{k}\right)
$$

então

$$
g^{*}\left(x_{i-1}\right)=g^{*}\left(x_{i}\right)=A v\left(\left\{x_{i-1}, x_{i}\right\}\right)
$$

e $g^{*}\left(x_{j}\right)=g\left(x_{j}\right)$, caso contrário.

4) Se esse novo conjunto de valores é não isotônico, então esse processo será substituído pelo passo 2 usando os novos valores e pesos, até se obter um conjunto isotônico de valores. O valor de $g^{*}\left(x_{i}\right)$ é a média ponderada do bloco no qual $x_{i}$ esteja contido.

\subsubsection{ENPMV sem erro de classificação para dados de status corrente}

Dados de status corrente surgem em situações onde o valor do tempo da variável T para ocorrência do evento é conhecida somente como sendo maior ou menor que o valor da variável observada $\mathrm{C}$.

Para uma determinada amostra $\left(C_{i}, \delta_{i}\right)$, para $i=1,2, \ldots, n$, a função de verossimilhança para $\mathrm{F}$ é dada por: 


$$
L(F)=\prod_{i=1}^{n}\left[F\left(C_{i}\right)\right]^{\delta_{i}}\left[1-F\left(C_{i}\right)\right]^{1-\delta_{i}},
$$

onde $\delta_{i}=I_{\left\{T_{i} \leq C_{i}\right\}}$ é a indicadora de censura à esquerda no ponto $C_{i}$. Com a aplicação do logaritmo, a função de log verossimilhança é dada por:

$$
l_{\delta}(F)=l(F ; \mathbf{C} ; \delta)=\sum_{i=1}^{n}\left[\delta_{i} \log F\left(C_{i}\right)+\left(1-\delta_{i}\right) \log \left(1-F\left(C_{i}\right)\right)\right] .
$$

Pode-se supor, sem perda de generalidade, que $\left(0 \leq C_{1} \leq C_{2} \leq \ldots \leq C_{n}\right)$. Aqui, $\mathbf{C}=\left(C_{1}, C_{2}, \ldots, C_{n}\right)$ e $\boldsymbol{\delta}=\left(\delta_{1}, \delta_{2}, \ldots, \delta_{n}\right)$.

Teorema 2.2.5. (Barlow et al., 1972 - Apêndice 1) O Teorema mostra que

$$
\psi(F)=\sum_{i=1}^{n}\left[\Phi\left(F\left(C_{i}\right)\right)+\left(g\left(C_{i}\right)-F\left(C_{i}\right)\right) \phi\left(F\left(C_{i}\right)\right)\right] w\left(C_{i}\right)
$$

com $\phi(y)=\frac{d \Phi}{d y}(y)$. Seja $\Phi$ uma função convexa que é finita no intervalo I com o dominio de variação da função g e infinito, caso contrário. A função (2.15) é maximizada em F pela regressão $g^{*} d a$ função $g$ com pesos $w$, ou seja, a função $g^{*}$ minimiza:

$$
S(F)=\sum_{i=1}^{n}\left[g\left(C_{i}\right)-F\left(C_{i}\right)\right]^{2} w\left(C_{i}\right)
$$

entre todas as funções isotônicas (isto é, não decrescentes) F que também maximiza $\psi$ em (2.15).

Pode-se observar que $l_{\delta}(F)$ em (2.14) pode ser escrito como o lado direito da equação (2.15), com $g\left(C_{i}\right)=\delta_{i}, w\left(C_{i}\right)=1$ e $\Phi\left(F\left(C_{i}\right)\right)=F\left(C_{i}\right) \log F\left(C_{i}\right)+(1-$ $\left.F\left(C_{i}\right)\right) \log \left(1-F\left(C_{i}\right)\right)$, para $i=1, \ldots, n$.

$\mathrm{Na}$ verdade,

$$
\begin{aligned}
\psi(F)= & \sum_{i=1}^{n}\left[F\left(C_{i}\right) \log \left(F\left(C_{i}\right)\right)+\left(1-F\left(C_{i}\right)\right) \log \left(1-F\left(C_{i}\right)\right)\right. \\
& \left.+\left(\delta_{i}-F\left(C_{i}\right)\right)\left(\log F\left(C_{i}\right)-\log \left(1-F\left(C_{i}\right)\right)\right)\right] \\
& =\sum_{i=1}^{n}\left[\delta_{i} \log F\left(C_{i}\right)+\left(1-\delta_{i}\right) \log \left(1-F\left(C_{i}\right)\right)\right]
\end{aligned}
$$


Portanto, o ENPMV $\hat{F}_{\delta}$ da função $\mathrm{F}$ nos pontos $C_{i}$ é dado pela função de regressão isotônica $g\left(C_{i}\right)=g_{i}=\delta_{i}$ com os pesos $w\left(C_{i}\right)=w_{i}=1$. Logo, o estimador ENPMV $\hat{F}_{\delta}$ da função $\mathrm{F}$ será também um estimador não paramétrico de mínimos quadrados ponderados da função F.

Uma forma prática de calcular $\hat{F}_{\delta}$, ou seja $\hat{F}_{\delta}\left(C_{i}\right)$, é dada pela inclinação do lado esquerdo de $\sum_{j=1}^{i} w_{j}=i$ da função minorante convexa máxima do diagrama das somas acumuladas dada pelos pontos $P_{i}=\left(\sum_{j=1}^{i} w_{j}, \sum_{j=1}^{i} g_{j} w_{j}\right)=\left(W_{i}, G_{i}\right)=$ $\left(i, \sum_{j=1}^{i} \delta_{j}\right)$, para $i=1, \ldots, n$ e $P_{0}=(0,0)$, conforme foi definido na seção 2.2 .2 e ilustrado no gráfico 2.1 .

Pode-se observar o diagrama da soma acumulada com sua função minorante convexa máxima apresentada na Figura 2.4.

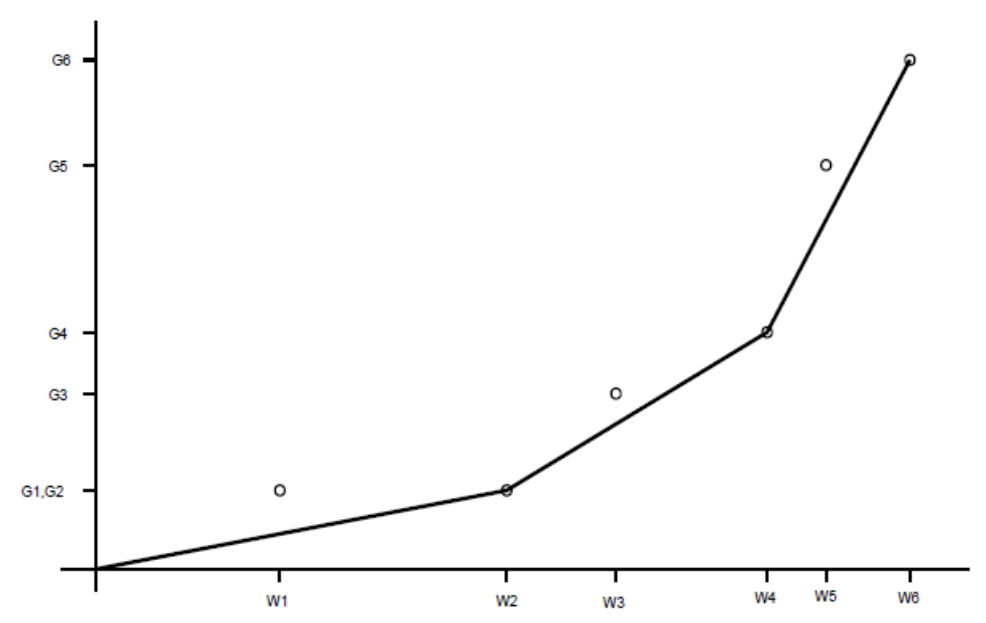

Figura 2.4: Diagrama das Somas Acumuladas (para os pontos $\left(W_{i}, G_{i}\right)$ ) e sua função minorante convexa máxima

A expressão matemática para $\hat{F}_{\delta}$ é dada pela fórmula max-min apresentada (em Barlow et al. 1972) conforme definido no Teorema 2.2.4 da Seção 2.2.3:

$$
\hat{F}_{\delta}\left(C_{i}\right)=\max _{j \leq i} \min _{k \geq i} \frac{\sum_{m=j}^{k} \delta_{m}}{k-j+1} .
$$




\subsection{Métodos para correção do ENPMV com erros de classificação}

Quando o tempo até a ocorrência de determinado evento de interesse é detectado através de exames clínicos, a identificação de tal evento fica sujeita a erros de classificação.

McKeown e Jewell (2010) estudaram as medidas de qualidade dos testes clínicos propondo um método para corrigir o ENPMV para dados de status corrente com erros de classificação.

Gomes e da-Silva (2011) propuseram também um método de estimação não paramétrico da função de distribuição do tempo de falha F levando em consideração o efeito da sensibilidade e especificidade do teste na determinação do valor de $\delta$ e consequentemente no cálculo do ENPMV da F.

Tal método envolve o uso de resultados da regressão isotônica presente em Barlow et al. (1972) e Robertson et al. (1998).

A variável indicadora $\delta$ utilizada para expressar censura à esquerda ou à direita da variável tempo de falha pode ser captada com erro de classificação, ou seja, uma consequência da sensibilidade ou especificidade do teste utilizado para determinar seu valor nos dados de status corrente.

O método iterativo e não paramétrico proposto para estimar a distribuição do tempo de falha leva exatamente em consideração esses erros de classificação devidos à sensibilidade e à especificidade do teste.

Em tal estudo, houve a indicação de que o método proposto reduz o vício consideravelmente, comparado ao ENPMV.

\subsubsection{ENPMV com erro de classificação}

Erros na classificação podem ocorrer na determinação dos valores da variável indicadora $\delta$ na presença de especificidade ou sensibilidade de um determinado teste clínico.

Na prática, observa-se uma variável indicadora positiva da variável resposta $\gamma_{i}$ em vez da variável $\delta_{i}$ (variável indicadora da verdadeira ocorrência de falha anterior 
ao instante de tempo $C_{i}$ ), para $i=1,2, \ldots, n$.

A sensibilidade e a especificidade foram definidas anteriormente, respectivamente, como as probabilidades condicionais:

$$
s=P(\gamma=1 \mid \delta=1)
$$

e

$$
e=P(\gamma=0 \mid \delta=0)
$$

Na presença de erro de classificação, o ENPMV $\hat{F}_{\gamma}$ de F, baseado na variável indicadora observada $\gamma$, é obtido pela maximização de log da verossimilhança da equação:

$$
l_{\gamma}(F)=l(F ; \mathbf{C} ; \gamma)=\sum_{i=1}^{n}\left[\gamma_{i} \log F\left(C_{i}\right)+\left(1-\gamma_{i}\right) \log \left(1-F\left(C_{i}\right)\right)\right]
$$

onde $\gamma=\left(\gamma_{1}, \ldots, \gamma_{n}\right)$.

De fato, nós tendemos a ter $\hat{F}_{\gamma}>\hat{F}_{\delta}$ na cauda à esquerda e $\hat{F}_{\gamma}<\hat{F}_{\delta}$ na cauda à direita de $F$. Isso acontece porque, na cauda à esquerda, nós esperamos ter mais mais observações com $\delta_{i}=0$ do que com $\delta_{i}=1$. E portanto, nós tendemos a ter mais erros de classificação com $\delta_{i}=0$ e $\gamma_{i}=1$ do que com $\delta_{i}=1$ e $\gamma_{i}=0$ (a menos que nós tenhamos $s$ muito maior que $e$, já que as probabilidades dos erros de classificação são 1-s e 1-e, respectivamente).

Por outro lado, na cauda à direita de $\mathrm{F}$, há a tendência de que a falha já tenha acontecido, caso do $\delta_{i}$ ser quase todo igual a $1\left(\delta_{i}=1\right)$. Então, na cauda à direita, nós esperamos ter mais observações com $\delta_{i}=1$ do que com $\delta_{i}=0$, fazendo os erros de classificação com $\delta_{i}=1$ e $\gamma_{i}=0$ serem mais frequentes que os com $\delta_{i}=0$ e $\gamma_{i}=1$.

Observando 2.14 e o procedimento de cálculo de $\hat{F}_{\gamma}$ e $\hat{F}_{\delta}$ descritos na Subseção 2.2.5, nós poderemos ver que devemos ter $\hat{F}_{\gamma}\left(C_{i}\right)>\hat{F}_{\delta}\left(C_{i}\right)$ para valores baixos de $C_{i}$ e o oposto, $\hat{F}_{\gamma}\left(C_{i}\right)<\hat{F}_{\delta}\left(C_{i}\right)$, para valores grandes de $C_{i}$.

A metodologia para obtenção da estimativa corrigida para a função do tempo de falha $\mathrm{F}$ através de $\hat{F}_{c}$, proposta por Gomes e da-Silva (2011), maximiza:

$$
E\left[l_{\delta}(F) \mid C, \gamma\right]=\sum_{i=1}^{n}\left\{E\left[\delta_{i} \mid \gamma_{i}\right] \log F\left(C_{i}\right)+\left(1-E\left[\delta_{i} \mid \gamma_{i}\right]\right) \log \left(1-F\left(C_{i}\right)\right)\right\}
$$


isto é,

$$
\hat{F}_{c}=\arg \max _{F \in \mathcal{F}}\left\{E\left[L_{\delta}(F \mid C, \gamma)\right]\right\}
$$

onde $\mathcal{F}=\{F:[0, \infty] \longrightarrow[0,1]: F$ é não decrescente $\}$.

Assume-se para isso que a especificidade e a sensibilidade não são dependentes do instante de tempo $C_{i}$ do teste de diagnóstico, embora o algoritmo possa ser utilizado quando estas dependem de $C_{i}$.

Então, pela aplicação do teorema 1.10 (Barlow et al., 1972 - Apêndice 1), os valores para $F\left(C_{i}\right)$ que maximizam (2.19) são dados pela função de regressão isotônica de:

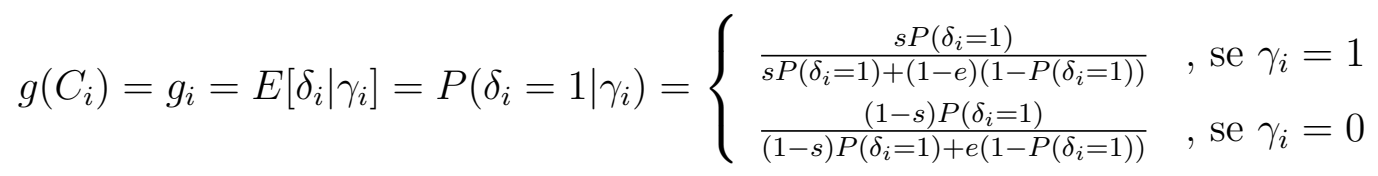

onde $s$ e $e$ são a sensibilidade e a especificidade do teste, respectivamente. Observe que $P\left(\delta_{i}=1 \mid \gamma_{i}=1\right)$ é o valor preditivo positivo (VPP) e $P\left(\delta_{i}=0 \mid \gamma_{i}=0\right)$ é o valor preditivo negativo (VPN) do teste.

Note que $P\left(\delta_{i}=1\right)=P\left(T_{i} \leq C_{i}\right)=F\left(C_{i}\right)$. Portanto, $E\left[\delta_{i} \mid \gamma_{i}\right]$ depende da função desconhecida $\mathrm{F}$ que queremos estimar. O método iterativo para calcular o ENPMV $\hat{F}_{c}$ de $\mathrm{F}$ é dado pelo algoritmo descrito a seguir:

Começamos pelo valor inicial de $P^{(0)}\left(\delta_{i}=1\right)=P^{(0)}\left(T_{i} \leq C_{i}\right)=F^{(0)}\left(C_{i}\right), \quad i=$ $1, \ldots, n$. Pode-se calcular inicialmente valores para $E^{(0)}\left[\delta i \mid \gamma_{i}\right]$ e usar esses valores para uma nova estimativa $F^{(1)}\left(C_{i}\right), \quad i=1, \ldots, n$, fazendo disso um processo iterativo para obter $\hat{F}_{c}$ :

\section{Algoritmo para o cálculo do ENPMV:}

1. Se $k=0$, e assumindo um valor pequeno para $\epsilon$ (a tolerância para o critério de parada), assumindo $s$ (sensibilidade) e $e$ (especificidade) faça os valores $P^{(k)}\left(\delta_{i}=1\right)=$ $P^{(k)}\left(T_{i} \leq C_{i}\right)=F^{(k)}\left(C_{i}\right), \quad i=1, \ldots, n ;$

2. Para as observações coletadas com $\gamma_{i}=1$, faça

$$
\boldsymbol{E}^{(k)}\left[\delta_{i} \mid \gamma_{i}=1\right]=P^{(k)}\left(\delta_{i}=1 \mid \gamma_{i}=1\right)=\frac{s F^{(k)}\left(C_{i}\right)}{s F^{(k)}\left(C_{i}\right)+(1-e)\left(1-F^{(k)}\left(C_{i}\right)\right)},
$$


Para as observações coletadas com $\gamma_{i}=0$, faça

$$
\boldsymbol{E}^{(k)}\left[\delta_{i} \mid \gamma_{i}=0\right]=P^{(k)}\left(\delta_{i}=1 \mid \gamma_{i}=0\right)=\frac{(1-s) F^{(k)}\left(C_{i}\right)}{(1-s) F^{(k)}\left(C_{i}\right)+e\left(1-F^{(k)}\left(C_{i}\right)\right)} ;
$$

3. Obtenha $F^{(k+1)}\left(C_{i}\right), \quad i=1, \ldots, n$ dada pela regressão isotônica da função $g^{(k)}\left(C_{i}\right)=g_{i}^{(k)}=\boldsymbol{E}^{(k)}\left[\delta_{i} \mid \gamma_{i}\right]$ e pesos $w^{k}\left(C_{i}\right)=w_{i}^{(k)}=1$, ou seja, $F^{(k+1)}\left(C_{i}\right)$ irá ser obtida pela inclinação no lado esquerdo de $\sum_{j=1}^{i} w_{j}^{(k)}=i$ da minorante convexa máxima do diagrama de somas acumuladas do maior minorante convexo com pontos dados por:

$$
P_{i}=\left(\sum_{j=1}^{i} w_{j}^{(k)}, \sum_{j=1}^{i} g_{j}^{(k)} w_{j}^{(k)}\right)=\left(i, \sum_{j=1}^{i} \boldsymbol{E}^{(k)}\left[\delta_{j} \mid \gamma_{j}\right]\right)
$$

4. Se $\max _{1 \leq i \leq n}\left|F^{(k+1)}\left(C_{i}\right)-F^{(k)}\left(C_{i}\right)\right|<\epsilon$, então pare e faça $\hat{F}_{c}\left(C_{i}\right)=F^{(k+1)}\left(C_{i}\right), \quad i=$ $1, \ldots, n$, caso contrário acrescente uma unidade ao indice k e volte ao passo 2 .

McKeown e Jewell (2010) também estudaram as medidas de qualidade dos testes clínicos propondo um método para corrigir o ENPMV para dados de status corrente com erros de classificação. Incorporaram tais medidas a um método não paramétrico considerando os indivíduos analisados apenas uma vez. Suas ideias foram aplicadas a um exemplo através do status de infecção papiloma vírus humano (HPV) de uma amostra de mulheres examinadas em São Francisco (E.U.A.).

McKeown e Jewell (2010) também utilizam-se das definições de tempo de falha (T) com função de distribuição $\mathrm{F}$, tempo de censura $(\mathrm{C})$ independente do $\mathrm{T}$, sensibilidade e especificidade para a solução do mesmo problema. Assumindo as verdadeiras probabilidades de erros de classificação $s, e>0,5$, sob esse modelo de erro de classificação, a função de verossimilhança (condicional) é dada por: 


$$
\prod_{i=1}^{n}\left[P\left(\gamma_{i}=1 \mid C_{i}\right)\right]^{\gamma_{i}}\left[P\left(\gamma_{i}=0 \mid C_{i}\right)\right]^{1-\gamma_{i}}
$$

onde

$$
\begin{gathered}
P\left(\gamma_{i}=1 \mid C_{i}\right)=P\left(\gamma_{i}=1 \mid \delta_{i}=1, C_{i}\right) P\left(\delta_{i}=1 \mid C_{i}\right)+P\left(\gamma_{i}=1 \mid \delta_{i}=0, C_{i}\right) P\left(\delta_{i}=0 \mid C_{i}\right) \\
=(s-1+e) F\left(C_{i}\right)+1-e
\end{gathered}
$$

e

$$
\begin{gathered}
P\left(\gamma_{i}=0 \mid C_{i}\right)=P\left(\gamma_{i}=0 \mid \delta_{i}=0, C_{i}\right) P\left(\delta_{i}=0 \mid C_{i}\right)+P\left(\gamma_{i}=0 \mid \delta_{i}=1, C_{i}\right) P\left(\delta_{i}=1 \mid C_{i}\right) \\
=e-(s-1+e) F\left(C_{i}\right)
\end{gathered}
$$

Para facilitar a notação, seja $\eta=s+e-1>0$. Então, a função de verossimilhança que admite erro de classificação na variável resposta pode ser escrita como:

$$
\prod_{i=1}^{n}\left[\eta F\left(C_{i}\right)+(1-e)\right]^{\gamma_{i}}\left[e-\eta F\left(C_{i}\right)\right]^{1-\gamma_{i}}
$$

com correspondente log-verossimilhança

$$
\sum_{i=1}^{n} \gamma_{i} \log \left(\eta F\left(C_{i}\right)+(1-e)\right)+\sum_{i=1}^{n}\left(1-\gamma_{i}\right) \log \left(e-\eta F\left(C_{i}\right)\right) .
$$

Escrevendo $G\left(C_{i}\right) \equiv \eta F\left(C_{i}\right)+(1-e)$, então a estimativa não-paramétrica da função de verossimilhança, quando os resultados do status corrente estão sujeitos a erros de classificação, pode ser encontrada através da obtenção de um vetor:

$$
\tilde{z}=\left(z_{1}=\hat{G}\left(C_{1}\right), \ldots, z_{n}=\hat{G}\left(C_{n}\right)\right) \in \mathbb{R}^{n}
$$

maximizando

$$
\phi\left(G\left(C_{i}\right)\right)=\sum_{i=1}^{n} \gamma_{i} \log \left(G\left(C_{i}\right)\right)+\sum_{i=1}^{n}\left(1-\gamma_{i}\right) \log \left(1-G\left(C_{i}\right)\right)
$$

sob a restrição:

$$
1-e \leq G\left(C_{1}\right) \leq G\left(C_{2}\right) \leq \ldots \leq G\left(C_{n}\right) \leq s
$$

Note que G é em si uma função de distribuição. 
Teorema 2.3.1. A identidade $z_{m}=\min \left(\max \left(\hat{z}_{m}, 1-e\right), s\right), m=1, \ldots, n$, define um vetor único, $\tilde{z}=\left(z_{1}, z_{2}, \ldots, z_{n}\right) \in \mathbb{R}^{n}$ maximizando (2.22) sob a restrição (2.23), com $G\left(C_{i}\right)$ substituído por $z_{i}$, onde

$$
\hat{z}_{m}=\max _{i \leq m} \min _{k \geq m} \frac{\sum_{i \leq j \leq k} \gamma_{j}}{k-i+1}
$$

é o Estimador Não-Paramétrico de Máxima Verossimilhança (ENPMV) não restrito da função de distribuição G baseada na verossimilhança (2.22), mas sem a restrição adicional (2.23).

Note que o vetor $\left(\hat{z}_{m}: m=1, \ldots, n\right)$ pode ser calculado usando o algoritmo padrão "pool-adjacent-violators" (PAV) em termos de minorante convexo. O vetor $\left\{z_{m}\right\}$ modifica qualquer valor de $\left\{\hat{z}_{m}\right\}$ menor que $1-e$ para igual a $1-e$ e similarmente modifica qualquer valor de $\left\{\hat{z}_{m}\right\}$ maior que s para igual a s. O ENPMV de F em um tempo de censura $C_{i}$ segue então utilizando a relação

$$
\hat{F}\left(C_{i}\right)=\frac{\left[\hat{G}\left(C_{i}\right)-1+e\right]}{\eta} .
$$

Prova do Teorema 2.3.1: primeiro, note que se $\gamma_{i}=0$ para $i=1,2, \ldots, k$ então maximizar (2.22) requer que o segundo termo seja tão grande quanto possível, nesse caso, definimos $z_{1}, z_{2}, \ldots, z_{k}=1-e$ sem afetar o problema de maximização sob o restante $z_{k+1}, \ldots, z_{n}$. Similarmente, se $\gamma_{i}=1$ para $j \leq i \leq n$, então para maximizar (2.22) devemos fazer o primeiro termo tão grande quanto possível, definindo $z_{j}, z_{j+1}, \ldots, z_{n}=s$.

Suponha que exista ao menos um $\gamma_{i}=1$ acompanhado por um $\gamma_{j}=0$, para algum $j>i$ (caso contrário, nós terminamos).

Seja ainda que $k_{0}$ ser o menor índice tal que $\gamma_{i}=1$ e seja $k_{1}$ ser o menor índice $k \geq k_{0}$ tal que

$$
\max _{i \leq m} \min _{k \geq m} \frac{\sum_{i \leq j \leq k} \gamma_{j}}{k-i+1} \geq 1-e .
$$

Analogamente, seja $m_{0}$ ser o menor índice $k \geq k_{1}$ tal que

$$
\max _{i \leq m} \min _{k \geq m} \frac{\sum_{i \leq j \leq k} \gamma_{j}}{k-i+1} \leq s,
$$

com $m_{1}$ sendo o maior índice i tal que $\gamma_{i}=0$. 
Então, $k_{0}$ e $m_{1}$ representa o índice do primeiro $\gamma_{i}=1$ e o último $\gamma_{j}=0$, respectivamente, onde $j>i$. Também, $k_{1}-1$ é o menor índice para o qual o ENPMV sem restrição não está abaixo de $1-e$, e $m_{0}$ é o maior índice para o qual o ENPMV sem restrição não está acima de s. A Figura 2.5 mostra como o posicionamento de tais índices apareceriam em termos de uma função de distribuição hipotética do ENPMV sem restriçao. As linhas tracejadas são posicionadas em $1-e$ e $s$, entre as quais o ENPMV sem restrição deve estar.

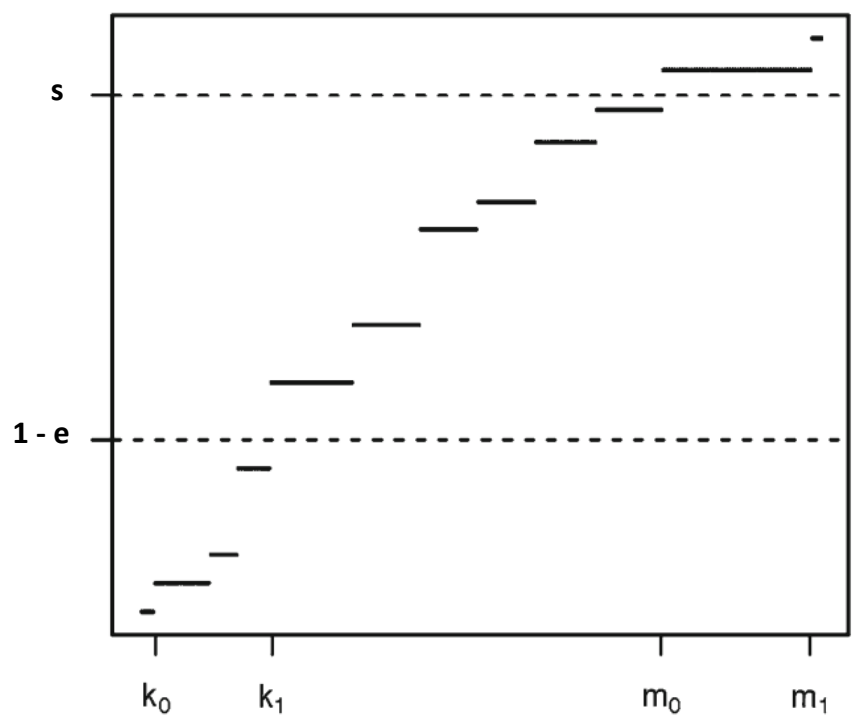

Figura 2.5: Hipotético ENPMV sem restrição, com as posições hipotéticas $s$ e $1-e$ mostradas no eixo vertical e os pontos $k_{0}, k_{1}, m_{0}$ e $m_{1}$ mostrados no eixo horizontal

Usando essas afirmações, o Teorema (2.3.1) pode ser escrita como:

(A) Para todos os índices $m<k_{1}, z_{m}=1-e$

(B) Para todos os índices $m>m_{0}, z_{m}=s$

(C) Para todos os índices $k_{1} \leq m \leq m_{0} ; z_{m}=\max _{i \leq m} \min _{k \geq m} \frac{\sum_{i \leq j \leq k} \gamma_{j}}{k-i+1}$, o ENPMV sem restrição.

Podemos provar a definição estabelecendo cada afirmação separadamente. Primeiro, mostramos que para todo índice $m<k_{1}, z_{m}=1-e$ maximiza os termos relevantes na verossimilhança (2.22), sujeito à restrição (2.23) sem afetar a função de otimização, ou restrição, baseada em $z_{i}$ para outros índices. Considere os índices $i$ para $k_{0} \leq i \leq k_{1}$. Suponha que os valores de $z_{i}$ sob este intervalo de índices assumem valores que aumentam e, necessariamente, $\geq 1-e$. Considere o maior desses índices 
(só para a "esquerda" de $k_{1}$ ) e os valores propostos maximizados de $z_{i}$ assumem o valor $(1-e+\epsilon)$, onde $\epsilon>0$. Não importa aqui se $z_{i}$ assume esse valor em um ou outro conjunto S de índices consecutivos. Suponha que entre o conjunto de índices, S, existem p índices $i$ onde $\gamma_{i}=1$ e $q$ índices onde $\gamma_{i}=0$. A contribuição para a verossimilhança (2.22) sob este conjunto de índices é por conseguinte:

$$
p \log (1-e+\epsilon)+q \log (e-\epsilon) \equiv h(\epsilon) .
$$

A derivada dessa função é

$$
h^{\prime}(\epsilon)=\left[\frac{p}{(1-e+\epsilon)}\right]-\left[\frac{q}{(e-\epsilon)}\right] .
$$

Agora, pela definição de $k_{1}$ relativa à definição do ENPMV sem restrição, segue-se

$$
\frac{p}{p+q}<1-e
$$

que por sua vez implica que

$$
\frac{q}{p}>\frac{e}{1-e}
$$

Já que $\epsilon>0$,

$$
\frac{e}{(1-e)}>\frac{(e-\epsilon)}{(1-e+\epsilon)},
$$

e disso então segue que $h^{\prime}(\epsilon)<0$ de forma que h é decrescente em $\epsilon$. Então, sem alterar o problema de otimização em termos de outros índices e restrições, podemos aumentar a verossimilhança pela redução do valor de $z_{i}$ proposto para o próximo valor inferior (à direita), onde $z_{j}=1-e+\lambda$ e $0<\lambda<\epsilon$. Entretanto, podemos repetir o mesmo argumento em termos de $\lambda$, e então continuar diminuindo os $z_{i}^{\prime} s$ relevantes até todos eles serem iguais a $(1-e)$. Isso prova $(\mathrm{A})$. Um argumento idêntico também estabelece (B). A afirmação (C) é consequência desde que

$$
z_{m}=\max _{i \leq m} \min _{k \geq m} \frac{\sum_{i \leq j \leq k} \gamma_{j}}{k-i+1}
$$

seja o ENPMV e atenda às restrições (2.23) pela definição de $k_{1}$ e $m_{0}$. A definição, portanto, está provada.

Tanto pelo método de Gomes e da-Silva (2011) quanto pelo método de McKeown e Jewell (2010), podemos obter também o EMV corrigido para dados de status corrente com erros de classificação para modelos paramétricos. 
Através da comparação dessas várias estimativas da função procurada, podese identificar a diminuição do vício nos estimadores através do método de correção proposto.

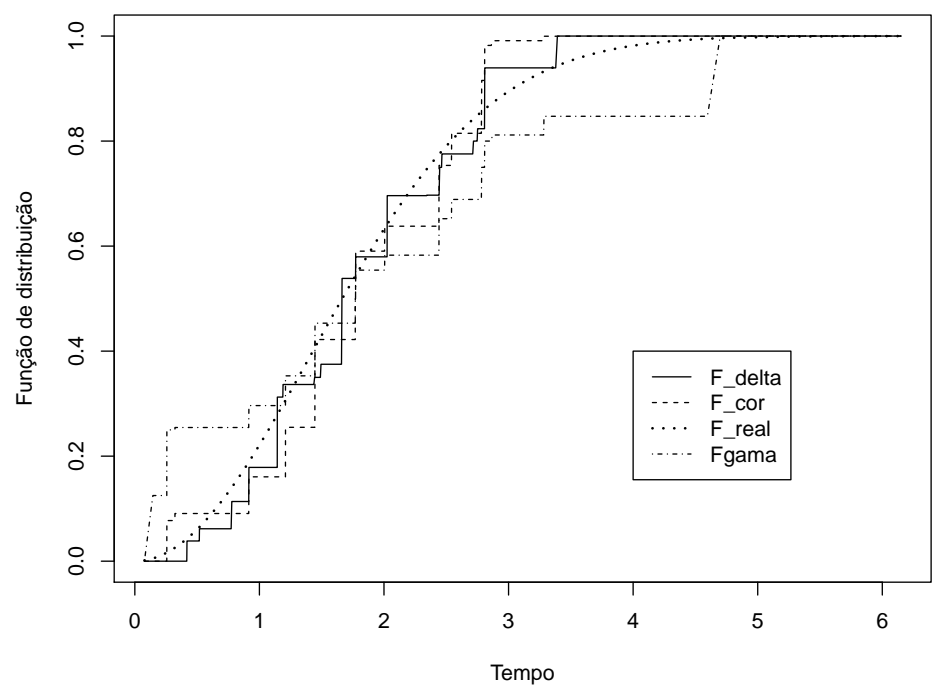

Figura 2.6: Diagrama das estimativas não paramétricas para os métodos propostos

Pelo diagrama das estimativas na Figura 2.6, pode-se perceber que $\hat{F}_{\delta}$ é o caso em que não há erro de classificação e sua estimativa foi muito próxima da curva real.

Para $\hat{F}_{\gamma}$, situação em que há vício forte nas caudas (com erro de classificação, ou seja, sem correção), percebe-se uma estimativa bastante distante da curva real.

Para $\hat{F}_{c}$, situação obtida através do método de correção para os erros de classificação, seu gráfico escada aparece muito próximo daquele que seria a curva sem erro de classificação, $\hat{F}_{\delta}$, ou bem próximo da curva real $\mathrm{F}$.

A Figura 2.7 mostra um exemplo de estimação paramétrica. Utilizou-se a função nlminb para maximização da função de verossimilhança para o caso da distribuição Weibull com parâmetro de forma igual a 2 e parâmetro de escala igual a 3. Foram geradas 1000 valores dessa distribuição.

Pelo diagrama das estimativas, pode-se perceber que para $\hat{F}_{\delta}$, caso em que não há erro de classificação, houve maior aproximação da curva real. Isso ocorre porque essa curva também é uma estimativa, estando mais próxima que a estimativa apresentada com erro (vide a seguir a tabela com as estimativas). 
Para $\hat{F}_{\gamma}$, situação em que há vício forte nas caudas (com erro de classificação e sem correção), percebe-se uma estimativa bastante distante da curva real. A estimativa sem correção apresenta um formato estranho.

Para $\hat{F}_{c}$, situação obtida através dos métodos de correção para os erros de classificação, seu gráfico aparece muito mais próximo daquele que seria a curva que não tivesse erro de classificação $\hat{F}_{\delta}$ ou bem próximo da curva real F.

Neste trabalho, abordaremos apenas a estimação não paramétrica da função de distribuição do tempo de falha.

Tabela 2.2: Valores reais dos parâmetros e estimativas corrigida, com erro de classificação e sem erro de classificação para a distribuição Weibull(2,3)

\begin{tabular}{l|r|r|r|r}
\hline$n=1000$ & real & corrigido & com erro de classif & sem erro de classif \\
\hline \hline par.forma & 2 & 2.116454 & 0.5385375 & 1.980686 \\
par.escala & 3 & 2.821738 & 3.9880818 & 2.917856 \\
\hline
\end{tabular}

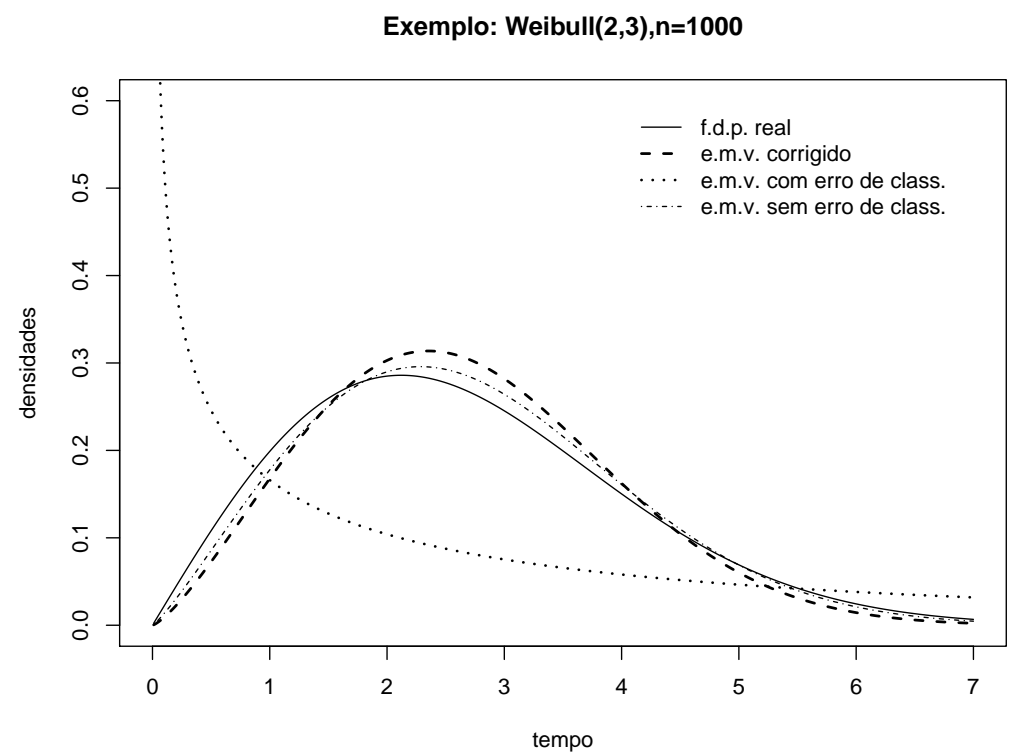

Figura 2.7: Diagrama das estimativas paramétricas para os métodos propostos 


\section{Capítulo 3}

\section{Núcleo-estimadores}

\subsection{Motivação para o método núcleo-estimadores}

Por muito tempo, diferentes formas para estimação de uma função de densidade têm sido propostas. Na metodologia clássica, utiliza-se alguma suposição sobre a forma funcional paramétrica dos dados. A partir da forma paramétrica suposta, estimam-se os parâmetros através dos dados (a máxima verossimilhança, por exemplo).

Todavia, muitas vezes, a suposição sobre a forma funcional paramétrica pode ser muito restritiva ou, em alguns casos, inadequada. Desta forma, os métodos de estimação de funções de densidade de probabilidade não paramétricos tornam-se necessários. Esta alternativa baseia-se na possibilidade de analisar os dados sem assumir uma forma funcional específica para a distribuição de probabilidade, fazendo-se suposições mais flexíveis a respeito da distribuição geradora dos dados observados.

\subsubsection{Estimação de densidades pelo método de núcleo-estima- dores (kernel)}

Para uma variável aleatória X com função de distribuição $\mathrm{F}$ absolutamente contínua temos uma função não negativa $\mathrm{f}$ tal que

$$
F(x)=P(X \leq x)=\int_{-\infty}^{x} f(t) d t \quad \forall x \in \Re .
$$


Neste caso, dizemos que f é a função densidade de probabilidade de X e deve satisfazer $\int_{-\infty}^{\infty} f(x) d x=1$.

A abordagem da estimação de funções de densidade através do método núcleoestimadores é a abordagem mais difundida.

Outros tipos de estimadores não-paramétricos de funções densidade incluem: histogramas, polígonos de frequência, splines, estimadores baseados em séries ortogonais e estimadores baseados em verossimilhança penalizada (Silverman, 1986; Scott, 1992; Simonoff, 1996). Pode-se ainda imaginar o estimador de kernel como uma generalização do histograma.

\subsubsection{Histogramas}

O método mais velho e mais amplamente utilizado para estimar uma densidade é o histograma. É um dos métodos não-paramétricos mais antigos de estimação de densidades.

A partir de uma origem $x_{0}$ e de um comprimento de intervalo h, definimos os retângulos do histograma como sendo os intervalos $\left[x_{0}+(r-1) h, x_{0}+r h\right)$ para valores inteiros positivos e negativos de r. Por definição, os intervalos devem ser escolhidos fechados à esquerda e abertos à direita.

O histograma é então definido por

$$
\hat{f}(x)=\frac{1}{n h} \times\left(\text { número de } X_{i}^{\prime} \text { s no mesmo intervalo que } x\right) .
$$

Para a construção do histograma, deve-se escolher a origem e o comprimento de intervalo, ou seja, o comprimento de intervalo controla (está diretamente relacionado) a magnitude do alisamento inerente ao procedimento.

O histograma pode ser generalizado pelo seguinte comprimento de intervalo variável:

$$
\hat{f}(x)=\frac{1}{n} \times \frac{\text { número de } X_{i}^{\prime} \text { s no mesmo intervalo que } x}{\text { comprimento do intervalo contendo } x}
$$

Empiricamente, o ponto principal é contar o número de observações que estarão contidas em cada intervalo. Sem perda de generalidade, seja o intervalo $\left[x-\frac{h}{2}, x+\frac{h}{2}\right)$. A probabilidade de uma observação pertencer a este intervalo é dado 
por

$$
P\left(X \in\left[x-\frac{h}{2}, x+\frac{h}{2}\right)\right)=\int_{x-\frac{h}{2}}^{x+\frac{h}{2}} f(t) d t,
$$

onde f é a densidade de X.

Logo, uma aproximação natural para esta probabilidade acima é

$$
P\left(X \in\left[x-\frac{h}{2}, x+\frac{h}{2}\right)\right) \approx \frac{1}{n} \#\left\{X_{i} \in\left[x-\frac{h}{2}, x+\frac{h}{2}\right)\right\} .
$$

Dessa forma, uma estimativa para f é

$$
\hat{f}(x)=\frac{1}{n h} \#\left\{X_{i} \in\left[x-\frac{h}{2}, x+\frac{h}{2}\right)\right\},
$$

onde este estimador não é contínuo e depende fortemente da escolha de h, conhecido também como parâmetro de suavização.

Ao variar o valor h obtêm-se diferentes formas de $\hat{f}_{h}(x)$. Para os extremos, ou seja, quando $h \rightarrow 0$, tem-se a representação muito ruidosa dos dados. Na situação oposta, quando $h \rightarrow \infty$, temos uma representação muito suave dos dados.

Apesar do histograma ser uma excelente ferramenta para representar os dados, é necessário considerar outras opções de estimativas da densidade.

A ideia do histograma permite encontrar um estimador de densidades mais geral, também conhecido como estimador naive (Silverman, 1986).

Da definição de densidade de probabilidade, considere:

Definição 3.1.1. Seja X uma v.a. com densidade f. Então,

$$
f(x)=\lim _{h \rightarrow 0} \frac{1}{2 h} P(x-h<X<x+h) .
$$

Para algum $h$ fixo, pode-se estimar $P(x-h<X<x+h)$ pela proporção de observações da amostra pertencentes ao intervalo $(x-h, x+h)$. Desse modo, um estimador natural $\hat{f}(x)$ de uma densidade é dado pela escolha de um $h$ pequeno, fazendo:

$$
\hat{f}(x)=\frac{1}{2 n h} \times \#\left\{X_{i} \in(x-h, x+h)\right\}, \text { para } i=1, \ldots, n .
$$

Essa expressão define o estimador naive.

Definição 3.1.2. Para expressar este estimador de forma mais clara, considere a função peso w:

$$
w(x)= \begin{cases}\frac{1}{2} & , \text { se }|x|<1 \\ 0 & , \text { caso contrário }\end{cases}
$$


Então, a estimativa para $f$ (o estimador naive) pode ser escrita como

$$
\hat{f}(x)=\frac{1}{n} \sum_{i=1}^{n} \frac{1}{h} w\left(\frac{x-X_{i}}{h}\right)
$$

A partir de (3.2) pode-se notar que o estimador (3.3) é construído colocandose um retângulo de largura $2 \mathrm{~h}$ e altura $(2 n h)^{-1}$ em cada observação e somando-os em seguida para se obter a estimativa $\hat{f}$.

Deve-se considerar o histograma construído a partir de dados e utilizar comprimentos de intervalo iguais a $2 \mathrm{~h}$. Deve-se assumir ainda que nenhum intervalo se sobrepõe a outro intervalo. Se $x$ for o centro de cada intervalo do histograma de (3.2), a estimativa naive $\hat{f}(x)$ será dada pelas ordenadas do histograma em $x$. Ou seja, a estimativa naive pode ser entendida como uma tentativa de se construir um histograma onde cada ponto é o centro do intervalo. A escolha do comprimento de intervalo será portanto determinada pelo parâmetro de suavização h (o qual controla a magnitude de suavização).

Note que $\hat{f}$ não é uma função contínua e tem derivada nula em todos os pontos exceto nos pontos de salto $x \pm h$. Além disso, o estimador de densidades baseado em uma função kernel é obtido substituindo-se a função peso $w$ por uma função nãonegativa $K$, denominada função kernel, satisfazendo a condição $\int_{-\infty}^{\infty} K(x) d x=1$.

Frequentemente, $K$ será uma função densidade de probabilidade simétrica (por exemplo, a função densidade de probabilidade normal ou a função peso $w$ utilizada na definição do estimador naive). Por analogia,

Definição 3.1.3. O estimador kernel é definido por

$$
\hat{f}(x)=\frac{1}{n h} \sum_{i=1}^{n} K\left(\frac{x-X_{i}}{h}\right),
$$

onde h é a 'janela do comprimento', também chamada de parâmetro de suavização, ou largura da banda para alguns autores.

\subsubsection{Estimação de Densidades Univariadas pelo Método Ker- nel}

No caso univariado, o estimador kernel para uma amostra aleatória $X_{1}, \ldots, X_{n}$ de observações independentes e identicamente distribuídas retirada de uma distribuição 
com densidade comum $f$, a qual será estimada, pode ser definido como:

$$
\hat{f}(x ; h)=\frac{1}{n h} \sum_{i=1}^{n} K\left(\frac{x-X_{i}}{h}\right)=\frac{1}{n} \sum_{i=1}^{n} K_{h}\left(x-X_{i}\right),
$$

onde h é o parâmetro de suavização, positivo e não-aleatório e $K$ é a função kernel, não-negativa, satisfazendo a condição $\int_{-\infty}^{\infty} K(x) d x=1$.

Vale destacar que a escolha da função kernel não é fundamental para a performance do método, sendo pois razoável escolher um kernel que auxilie na eficiência computacional (Silverman 1986; Epanechnikov 1969).

Tabela 3.1: Funções kernel comumente utilizadas com dados univariados

\begin{tabular}{l|c}
\hline Função kernel & Forma analítica, $K(x)$ \\
\hline Retangular & $\frac{1}{2}$, para $|\mathrm{x}|<1,0$ caso contrário \\
Triangular & $1-|x|$, para $|x|<1,0$ caso contrário \\
Biweight & $\frac{15}{16}\left(1-x^{2}\right)^{2}$, para $|x|<1,0$ caso contrário \\
Normal & $\frac{1}{\sqrt{2 \pi}} \exp \left(\frac{-x^{2}}{2}\right)$, para $-\infty<x<\infty$ \\
Epanechnikov & $\frac{3}{4} \frac{\left(1-\frac{x^{2}}{5}\right)}{\sqrt{5}}$, para $|x|<\sqrt{5}, 0$ caso contrário \\
\hline
\end{tabular}

A Figura 3.1 a seguir apresenta a estimativa da densidade univariada pelo método kernel. A linha sólida representa a densidade estimada e as linhas tracejadas representam as funções kernel individuais. A amostra é composta pelos valores $X_{1}=$ -1.0, $X_{2}=-0.8, X_{3}=-0.6, X_{4}=0.5, X_{5}=1.2$ e a função kernel é a função gaussiana $\mathrm{N}(0,1)$. Vale ainda destacar que na prática, a densidade estimada envolve um número muito maior de observações.

Nesse exemplo dado, $K_{h}$ é simplesmente a densidade $N\left(0, h^{2}\right)$.

Basicamente, a estimativa kernel $\hat{f}$ depende dos dados coletados, bem como da janela de amplitude. Essa dependência não irá geralmente ser expressa explicitamente. Para cada $x, \hat{f}(x)$ pode ser pensada como sendo uma variável aleatória, por causa da dependência das observações $X_{1}, \ldots, X_{n}$. Qualquer uso de probabilidade, esperança e variância envolvendo $\hat{f}$ será referente à distribuição amostral como uma estatística dessas observações aleatórias. 


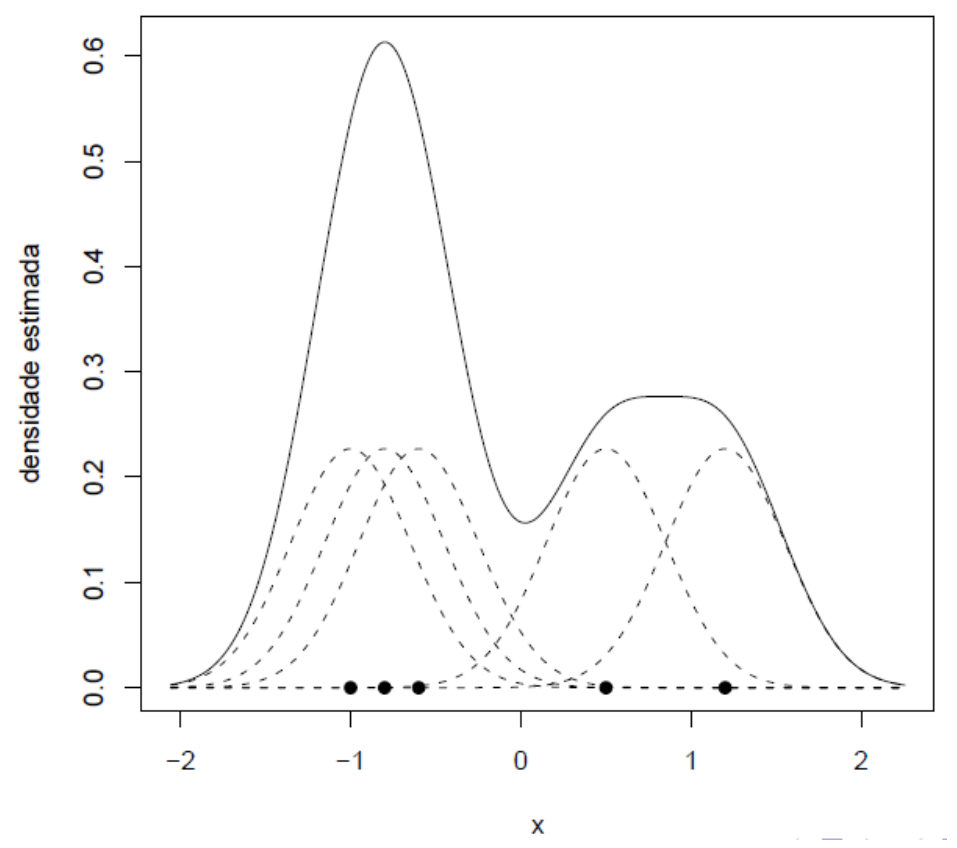

Figura 3.1: Estimativa da densidade univariada pelo método kernel, $(-)$ densidade estimada e (- - - -) funções kernel individuais.

\subsubsection{Estimadores de função peso geral}

A classe de estimadores de função peso geral é uma classe que inclui vários outros estimadores. Supondo uma função de dois $\operatorname{argumentos} w(x, y)$, a função peso irá satisfazer à condição (em muito dos casos):

$$
\int_{-\infty}^{+\infty} w(x, y) d y=1 \text { e } w(x, y) \geq 0 \text { para todo } x \text { e } y \text {. }
$$

Pode-se entender que $w$ é definido de tal forma que muitos dos pesos da densidade de probabilidade $w(x, \cdot)$ estão próximos de $x$.

Definição 3.1.4. Uma estimativa da densidade a partir dos dados pode ser obtida utilizando-se:

$$
\hat{f}(t)=\frac{1}{n} \sum_{i=1}^{n} w\left(X_{i}, t\right),
$$

sendo essa portanto a definição dos estimadores da função peso geral.

Essas duas condições, (3.6) e (3.7), garantem que $\hat{f}$ seja uma função de densidade de probabilidade e as propriedades de suavização da $\hat{f}$ sejam inerentes à função peso $w(x, \cdot)$. Essa classe de estimadores (estimadores da função peso geral) pode ser analisada de duas formas: primeiro, como sendo um conceito único o qual torna 
possível, por exemplo, obter resultados teóricos aplicáveis a todo tipo de estimadores aparentemente distintos. Segundo, é possível definir estimadores úteis que não se classifiquem entre os estimadores mais conhecidos, mas que estão restritos à fórmula (3.7).

Para obter o histograma como um caso especial de (3.7), utiliza-se:

$$
w(x, y)= \begin{cases}\frac{1}{h(x)} & , \text { se } x \text { e } y \text { estiverem no mesmo intervalo } \\ 0 & , \text { caso contrário. }\end{cases}
$$

onde $h(x)$ é o comprimento do intervalo contendo $x$.

O estimador de Kernel pode ser então obtido colocando-se:

$$
w(x, y)=\frac{1}{h} K\left(\frac{y-x}{h}\right) .
$$

Uma forma de lidar com alguns tipos de dados é utilizar uma função peso que seja, para cada $x$ fixo, uma densidade de probabilidade definida positivamente (parte positiva da curva) e que contenha a massa de probabilidade concentrada em torno de $x$.

\subsection{Medidas de discrepância: erro quadrático mé- dio e o erro quadrático médio integrado}

Várias medidas são estudadas para medir a discrepância entre a densidade estimada $\hat{f}$ e a verdadeira densidade $f$. Quando considera-se a estimação de um simples ponto, uma medida natural de discrepância a ser utilizada é o erro quadrático médio (EQM), definida como:

$$
\operatorname{EQM}_{x}(\hat{f})=\mathrm{E}\{\hat{f}(x)-f(x)\}^{2}
$$

e pela propriedade padrão da média e da variância, tem-se:

$$
\operatorname{EQM}_{x}(\hat{f})=\{\mathrm{E} \hat{f}(x)-f(x)\}^{2}+\operatorname{var} \hat{f}(x)
$$

respectivamente, o quadrado do vício e a variância de $x$. Poderemos ver que existe um trade-off entre o viés e a variância, ou seja, o viés pode ser reduzido com o prejuízo do aumento da variância e vice-versa, dependendo da suavização. 
A primeira (Rosenblatt, 1956) e mais amplamente utilizada forma de substituir a medida de precisão global de $\hat{f}$ é o erro quadrático médio integrado (EQMI) definido como:

$$
\operatorname{EQMI}(\hat{f})=\mathrm{E} \int\{\hat{f}(x)-f(x)\}^{2} d x \text {. }
$$

Embora existam outras medidas globais de discrepância, o EQMI é considerado a medida global mais utilizada.

Vale notar que se o termo a ser integrado for não-negativo, o EQMI possui a forma alternativa:

$$
\begin{aligned}
& \operatorname{EQMI}(\hat{f})=\int \mathrm{E}\{\hat{f}(x)-f(x)\}^{2} d x \\
&= \int \operatorname{EQM}_{x}(\hat{f}) d x \\
&=\int\{\mathrm{E} \hat{f}(x)-f(x)\}^{2}+\int \operatorname{var} \hat{f}(x) d x
\end{aligned}
$$

expressão que fornece o EQMI como sendo uma soma do viés quadrático integrado e da variância integrada.

\subsubsection{Propriedades elementares de uma amostra infinita}

Supondo que $\hat{f}$ seja uma estimativa de função peso geral, conforme definido em (3.7), através de uma manipulação elementar (Whittle, 1958), conclui-se que, para cada $t$,

$$
\mathrm{E} \hat{f}(t)=\frac{1}{n} \sum \mathrm{E} w\left(X_{i}, t\right)=\int w(x, t) f(x) d x
$$

e, desde que $X_{i}$ sejam independentes,

$$
\operatorname{var} \hat{f}(t)=\frac{1}{n} \operatorname{var} w\left(X_{i}, t\right)=\frac{1}{n}\left[\int w(x, t)^{2} f(x) d x-\left\{\int w(x, t) f(x) d x\right\}^{2}\right]
$$

As expressões do EQM e EQMI podem ser obtidas substituindo-se essas fórmulas em (3.13). Uma propriedade interessante de (3.14) é que, dado uma $f$, o vício

$\mathrm{E}\{\hat{f}(t)-f(t)\}$ não depende diretamente da amostra, mas depende somente da função peso. Isso é importante conceitualmente pelo fato de mostrar que por maior que seja a amostra, não necessariamente haverá a redução do viés. Será portanto necessário ajustar uma função peso para obter estimativas assintoticamente não viesadas. 


\subsection{Suavização do ENPMV da função de distribui- ção acumulada do tempo de falha para dados de status corrente}

Note que

$$
\begin{gathered}
\tilde{F}(t)=\int_{-\infty}^{t} \hat{f}_{h}(u) d u=\frac{1}{n h} \sum_{i=1}^{n} \int_{-\infty}^{t} k\left(\frac{u-X_{i}}{h}\right) d u=\frac{1}{n} \sum_{i=1}^{n} K\left(\frac{t-X_{i}}{h}\right)= \\
=\sum_{i=1}^{n} K\left(\frac{t-X_{(i)}}{h}\right)\left[\hat{F}\left(X_{(i)}\right)-\hat{F}\left(X_{(i-1)}\right)\right]=\int_{-\infty}^{\infty} K\left(\frac{t-s}{h}\right) d \hat{F}(s),
\end{gathered}
$$

onde $\hat{F}$ é a função de distribuição acumulada empírica e $X_{(i)}, i=1, \ldots, n$ são os pontos de salto de $\hat{F}$. Para a suavização da função de distribuição acumulada em que os saltos apresentam magnitudes distintas, como é o caso em que há presença de censura,

$$
\tilde{F}(t)=\int_{-\infty}^{\infty} K\left(\frac{t-s}{h}\right) d \hat{F}(s)=\sum_{i=1}^{n} K\left(\frac{t-C_{i}}{h}\right)\left[\hat{F}\left(C_{i}\right)-\hat{F}\left(C_{i-1}\right)\right]
$$

onde $K(x)=\int_{-\infty}^{x} k(u) d u$.

Para dados de status corrente, utilizamos $h=\frac{a}{6} \frac{1.1}{n^{1 / 5}}$, onde $a$ é a amplitude dos pontos de salto, isto é, $C_{n}-C_{1}$, conforme sugerido em Douglas e Cohen (2001). 


\section{Capítulo 4}

\section{Simulação e análise dos resultados}

\subsection{Integração com eliminação de parâmetros in- covenientes}

A eliminação de parâmetros inconvenientes é um problema comum na inferência estatística e tem sido formalmente estudada por vários autores.

Berger et al. (1999) descrevem situações em que se faz necessária a eliminação de parâmetros sobre os quais não se tem interesse em fazer inferência.

Em nosso caso, queremos encontrar a função de distribuição F do tempo de falha que maximiza $L(F, s, e)$ dada por (2.21) quando não sabemos os valores de $s$ e $e$. Uma maneira de fazer isso é gerar m valores de $s$ e $e$ a partir de uma distribuição de probabilidade, calcular $\hat{F}^{(j)}, j=1, \ldots, m$, que maximiza (2.21) para cada par de valores de $\left(s_{j}, e_{j}\right)$ gerados, e tomar

$$
\hat{F}(t)=\frac{1}{m} \sum_{j=1}^{m} \hat{F}^{(j)}(t):
$$

Isso equivale a aproximar o cálculo de $\hat{F}(t)$ dado por (4.1).

$$
\hat{F}(t)=\iint_{s, e} \hat{F}(t \mid s, e) \pi(s, e) d s d e
$$

Para o nosso estudo de simulação, consideramos a sensibilidade e a especificidade provindas de uma distribuição Beta(a,b) reescalonada para ter suporte no intervalo $[0.5,1]$ : 


$$
X \sim \operatorname{Beta}(\mathrm{a}, \mathrm{b}) \Rightarrow Y=\frac{X+1}{2} \Rightarrow Y \in[0.5,1]
$$

com,

$$
\begin{gathered}
E(Y)=\frac{E(X)+1}{2}=\frac{\frac{a}{a+b}+1}{2}=\frac{2 a+b}{2(a+b)}=\mu, \\
\operatorname{Var}(Y)=\frac{1}{4} \operatorname{Var}(X)=\frac{a b}{4(a+b)^{2}(a+b+1)}=\sigma^{2} \Rightarrow \\
a=(2 \mu-1)\left[\frac{(2 \mu-1)(1-\mu)}{\sigma^{2}}-1\right]
\end{gathered}
$$

e

$$
b=2(1-\mu)\left[\frac{(2 \mu-1)(1-\mu)}{\sigma^{2}}-1\right]
$$

Assim, fixando-se valores para a média e o desvio-padrão da sensibilidade e especificidade, obtêm-se os valores dos parâmetros para a distribuição Beta que assumimos descrever nossa incerteza sobre os reais valores desconhecidos de $s$ e $e$.

Note-se que poderia ser obtido intervalo de confiança para $F$ com base nas m estimativas corrigidas tomando-se os quantis de ordem $\frac{\alpha}{2}$ e $1-\frac{\alpha}{2}$ de $\hat{F}^{(j)}(t)$, para $j=1, \ldots, m$. Este intervalo refletiria o efeito de nossa incerteza sobre valores de $s$ e $e$ na estimativa de $\mathrm{F}$.

Uma ilustração do uso desta metodologia está descrito na Figura 4.1, com $\mu=0.8$ e $\sigma=0.1$.

\subsection{Simulação e análise dos resultados}

Nesta parte, são descritas as simulações computacionais realizadas via software R (versão 3.0.1) com os resultados numéricos obtidos (programação no Apêndice 2).

Para avaliação do vício do ENPMV, foram utilizados valores fixos para a sensibilidade real e para a especificidade real. Além disso, a distribuição do tempo de falha e do tempo de censura utilizada foi a distribuição Weibull $(2,2)$, com parâmetro de forma igual a 2, parâmetro de escala igual a 2, resultando assim a variável de censura $C$, com $50 \%$ de censura à direita e $50 \%$ de censura à esquerda. 


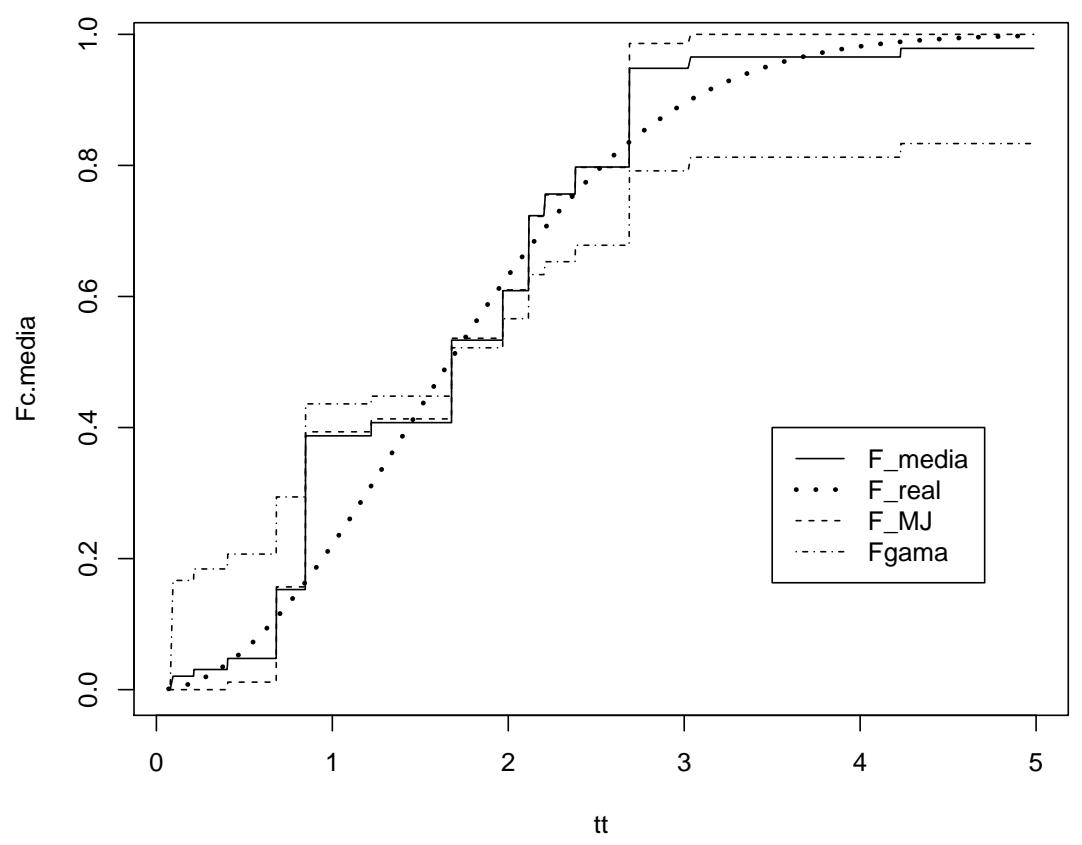

Figura 4.1: Gráfico da média das estimativas a partir do uso de distribuições a priori para sensibilidade e especificidade assumidas

Já no exemplo ilustrado na Figura 4.2 , utilizou-se um valor real de 0.8 tanto para a sensibilidade quanto para a especificidade, obtendo $\hat{F}_{\gamma}$ e $\hat{F}_{\delta}$ através do cálculo do estimador não paramétrico de máxima verossimilhança (ENPMV) da função de distribuição do tempo de falha, além da função corrigida, $\hat{F}_{c o r}$, através do método proposto por Gomes e da-Silva (2011). Mais ainda, foi calculada a função de distribuição do tempo de falha pelo método proposto por McKeown e Jewell (2010), a $\hat{F}_{M J}$, conforme mostrados na Figura 4.2.

A avaliação do vício e o erro-padrão das estimativas $\hat{F}_{\gamma}$ e $\hat{F}_{c}$ foram feitos nos quantis de ordem $0.10,0.25,0.50$ e 0.75 da distribuição do tempo de falha.

Também foram feitas algumas escolhas empíricas dos valores para o parâmetro de escala e de forma para as distribuições das variáveis do tempo de falha e do tempo de censura, permitindo também o estudo de diferentes níveis de proporções de censuras. Além disso, foram feitas combinações dos níveis de sensibilidade e especificidade, para melhor análise do método de estimação proposto.

Para a escolha do parâmetro de escala e de forma iguais a 2 da distribuição 


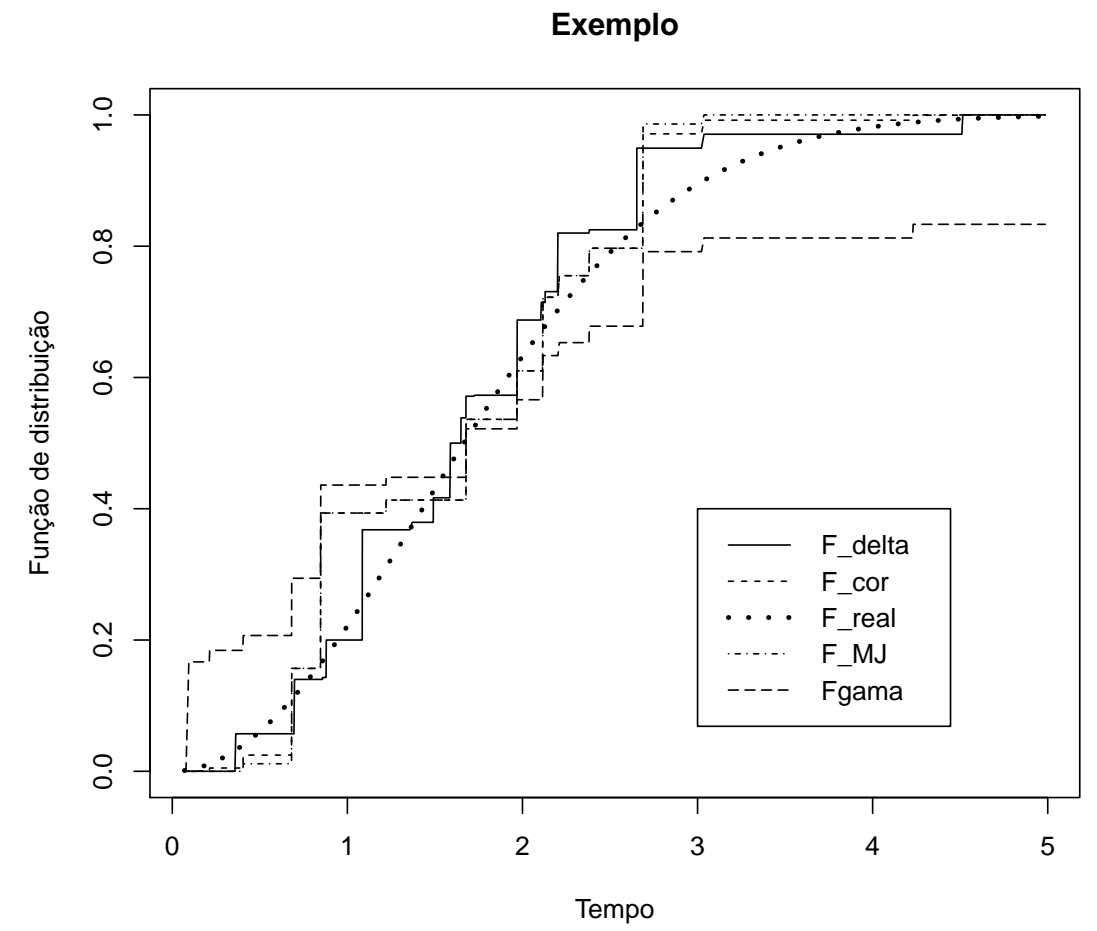

Figura 4.2: Gráfico da função de distribuição do tempo de falha, com $50 \%$ de censura à esquerda e 50\% de censura à direita

Weibull(a,b) para ambas as variáveis de tempo de falha e de tempo de censura, obtémse a proporção de $50 \%$ de censura à esquerda e 50\% de censura à direita.

Para a variável do tempo de falha com parâmetro de escala igual a 2 e parâmetro de forma igual a 2 e para a variável do tempo de censura com parâmetro de escala igual a 2 e parâmetro de forma igual a 3 obteve-se o percentual de $70 \%$ de censura à esquerda e 30\% de censura à direita.

Por fim, para a variável do tempo de falha com parâmetro de escala igual a 2 e parâmetro de forma igual a 3 e para a variável do tempo de censura com parâmetro de escala igual a 2 e parâmetro de forma igual a 2 obteve-se o percentual de $30 \%$ de censura à esquerda e $70 \%$ de censura à direita. Tal simulação foi considerada tanto para versões das estimativas originais quanto para as estimativas suavizadas, com diferentes tamanhos de amostras $(n=200 ; n=500$ ou $n=1000)$, resultando ao todo em 18 tabelas para estes 3 cenários de proporções de censuras.

Foram geradas 5000 amostras para cada combinação de tamanho de amostra, proporção de censura à esquerda, valores de sensibilidade e especificidade. 
Observando ainda essas 18 tabelas, podemos verificar que em geral a performance da função de distribuição estimada corrigida $\hat{F}_{c o r}$ é melhor que a performance da estimativa da função de distribuição não corrigida $\hat{F}_{\gamma}$.

Em relação à variabilidade, para o tamanho de amostra $(n=200)$, por exemplo, as Tabelas 4.1, 4.4, 4.7, 4.10, 4.13, 4.16 mostram que o erro padrão da distribuição estimada $\hat{F}_{c o r}$ e $\hat{F}_{\gamma}$ na cauda da distribuição de $\mathrm{T}$ depende da proporção de censura utilizada no estudo, tanto para as estimativas da $\hat{F}_{c o r}$ e $\hat{F}_{\gamma}$ sem suavização quanto para as estimativas com suavização via Kernel.

Percebemos ainda que, em geral, tanto para as tabelas compostas por estimativas que não utilizaram suavização via kernel, (Tabelas: 4.1, 4.2, 4.3, 4.7, 4.8, 4.9, $4.13,4.14,4.15)$, quanto para as tabelas compostas por estimativas que a utilizaram, (Tabelas: 4.4, 4.5, 4.6, 4.10, 4.11, 4.12, 4.16, 4.17, 4.18), independentemente da proporção de censura avaliada (censura à esquerda de $50 \%$, de $70 \%$, ou de $30 \%$ ), o erro padrão $\hat{\sigma}_{c o r}$ é ligeiramente maior que o erro padrão $\hat{\sigma}_{\gamma}$. Entretanto, essa diferença é compensada pela grande diferença no viés dos dois estimadores, mostrando a maior proximidade da estimativa $\hat{F}_{c o r}$ em relação a $F_{\text {real }}$ que a estimativa da $\hat{F}_{\gamma}$.

\subsubsection{Avaliação do vício em função da sensibilidade e espe- cificidade}

Os gráficos a seguir foram criados para avaliar o vício de $\hat{F}_{\gamma}$ como função da sensibilidade e especificidade nos percentis 10, 25, 50 e 75. A simulação foi relacionada ao percentual de $50 \%$ de censura à esquerda e $50 \%$ de censura à direita com tempo de falha e de censura com distribuição Weibull, ambas com parâmetro de forma e parâmetro de escala iguais a 2 .

O Gráfico 4.3, no primeiro decil, permite identificar que o maior vício alcançado é para valores da sensibilidade iguais a 1 e especificidade iguais a 0.5 da distribuição $\hat{F}_{\gamma}$, com o vício de 0.46 , aproximadamente. De fato, podemos perceber que o vício é nulo quando a sensibilidade e a especificidade assumem valores iguais a 1. Quando a sensibilidade e a especificidade assumem ambos os valores iguais a 0.5, o efeito gerado no erro que está se comentendo é muito alto e próximo a 0.38 .

O Gráfico 4.4, no percentil 25, revelou que o maior vício alcançado é para 


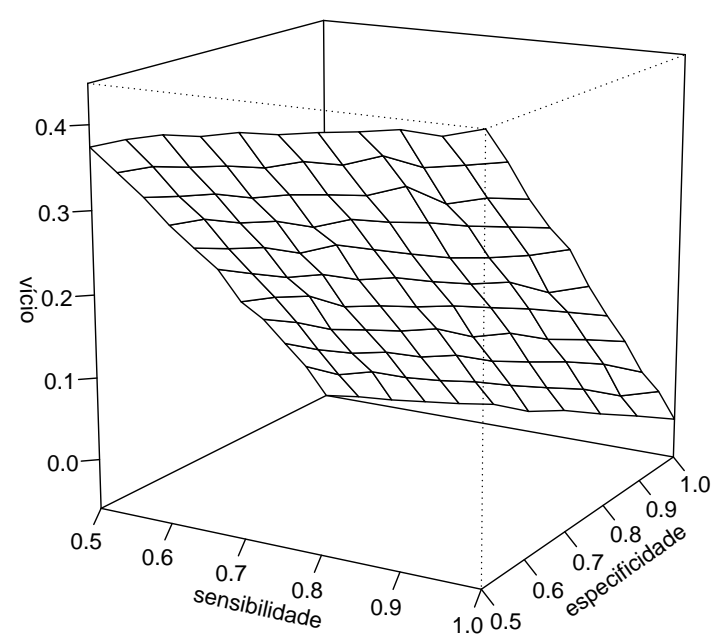

Figura 4.3: Gráfico do vício em função da sensibilidade e especificidade, avaliado no $1^{\circ}$ decil, com $50 \%$ de censura à esquerda e $50 \%$ de censura à direita

10. quartil

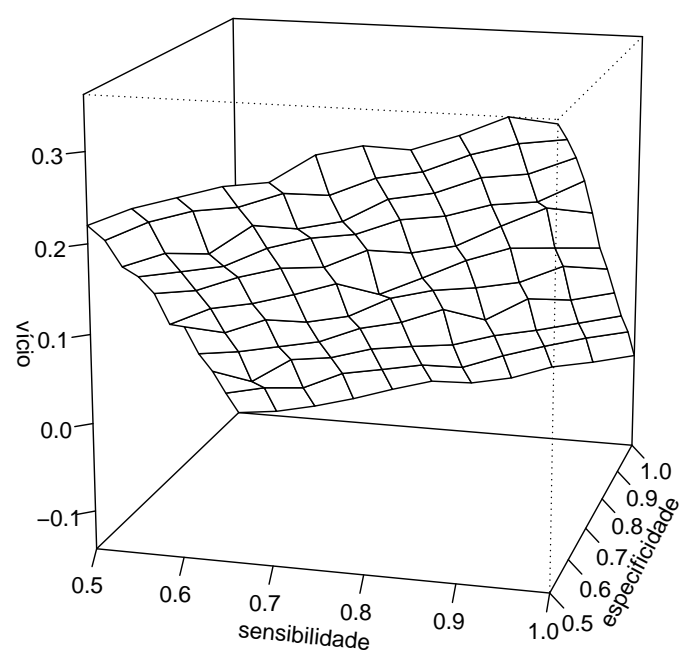

Figura 4.4: Gráfico do vício em função da sensibilidade e especificidade, avaliado no $1^{\circ}$ quartil, com $50 \%$ de censura à esquerda e $50 \%$ de censura à direita

valores da sensibilidade iguais a 1 e especificidade iguais a 0.5 da distribuição $F_{\gamma}$, com o vício de 0.37 , aproximadamente. Quando a sensibilidade e a especificidade assumem 
ambos os valores iguais a 0.5 , o efeito gerado no erro que está se comentendo é também muito alto e próximo a 0.22 .

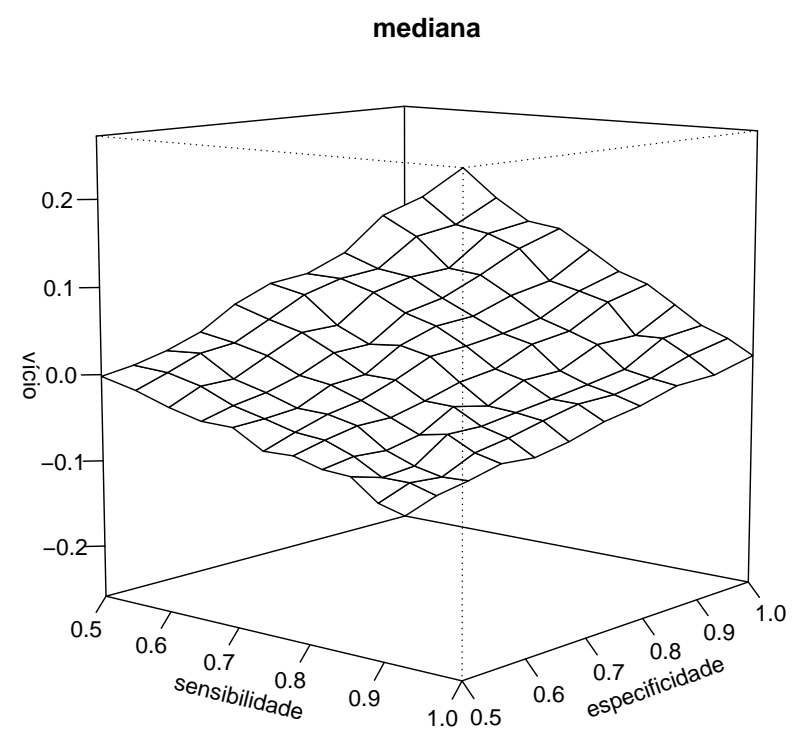

Figura 4.5: Gráfico do vicio em função da sensibilidade e especificidade, avaliado na mediana, com 50\% de censura à esquerda e 50\% de censura à direita

O Gráfico 4.5, na mediana, permite perceber uma característica importante e interessante a respeito do efeito da sensibilidade e especificidade no erro que se está cometendo. O vício é nulo para os valores da sensibilidade iguais aos valores da especificidade, ou seja, a relação $s=e$. Para essa reta, percebe-se que não há vício na distribuição $\hat{F}_{\gamma}$. Os maiores erros são cometidos quando a especificidade assume valor igual a 1 e a sensibilidade valor igual a 0.5 ou vice-versa.

E por último, o Gráfico 4.6, referente ao $3^{\circ}$ quartil, revelou que o maior vício alcançado é para valores da sensibilidade iguais a 1 e especificidade iguais a 0.5 da distribuição $\hat{F}_{\gamma}$, com o vício positivo de 0.13 , aproximadamente. Quando a sensibilidade e a especificidade assumem ambos os valores iguais a 0.5 , o efeito gerado no erro que está se comentendo é também muito alto e próximo a -0.22 . Da mesma forma, quando especificidade assume o valor igual a 1 e a sensibilidade o valor igual a 0.5 o efeito sobre o erro cometido é o maior de todos, próximo a -0.4 . 


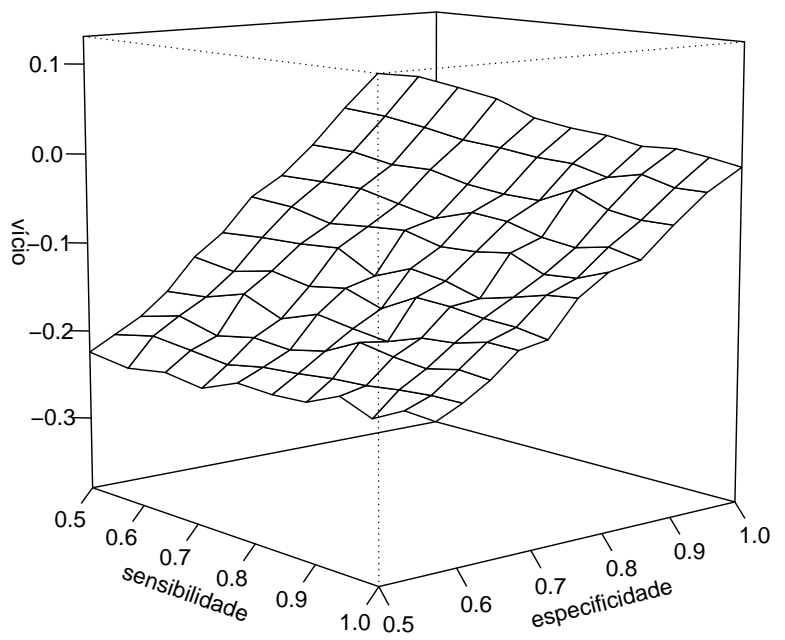

Figura 4.6: Gráfico do vício em função da sensibilidade e especificidade, avaliado no $3^{\circ}$ quartil, com $50 \%$ de censura à esquerda e $50 \%$ de censura à direita

\subsubsection{Versões suavizadas da função de distribuição}

As curvas ilustrativas dadas a seguir, Figura 4.7, são para estimativas suavizadas, via método de kernel, da função de distribuição da $\hat{F}_{\gamma}$, da $\hat{F}_{\delta}$ e da $\hat{F}_{c o r}$.

A partir do Gráfico 4.7 percebe-se que a estimativa $\hat{F}_{c o r}$ está mais próxima da curva real que a estimativa $\hat{F}_{\gamma}$.

Esse estudo de simulação para as estimativas suavizadas da função de distribuição do tempo de falha da $\hat{F}_{\gamma}, \hat{F}_{\delta}$ e $\hat{F}_{\text {cor }}$ permitiu identificar que o método proposto reduz o vício substancialmente quando comparado com o ENPMV. Ou seja, percebese que ao suavizar a função de distribuição do tempo de falha há de fato uma redução no vício indicando que a suavização permite uma aproximação ainda maior da curva estimada corrigida com a curva real.

Pode-se concluir, por meio das análises das simulações que a versão corrigida $\hat{F}_{c o r}$ do ENPMV da F apresenta uma diminuição significativa do viés em relação a $\hat{F}_{\gamma}$. Também pode-se verificar que o erro padrão da distribuição $F$ diminui consideravelmente quando o tamanho da amostra cresce, permitindo assim, a melhora significativa das estimativas da F, especialmente para valores mais baixos da sensibilidade e espe- 


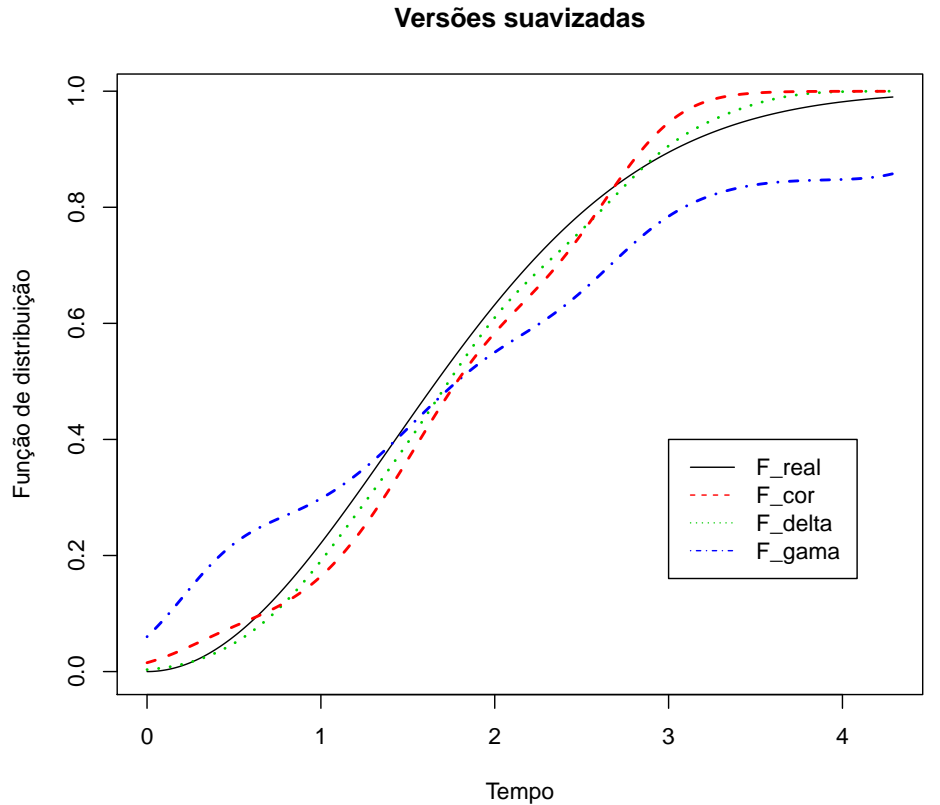

Figura 4.7: Gráfico ilustrativo para estimativas suavizadas da função de distribuição do tempo de falha da $\hat{F}_{\gamma}, \hat{F}_{\delta}$ e $\hat{F}_{c o r}$

cificidade.

\subsubsection{Aplicação para um conjunto de dados reais}

A aplicação para um conjunto de dados reais utilizado foi feita para os dados utilizados no estudo de Gomes e da-Silva (2011), obtido através do estudo de Criqui (1985) que verificou o efeito da sensibilidade e da especificidade na avaliação clínica de doença arterial periférica. Nesta parte, as estimativas de sensibilidade e especificidade foram de 0.712 e 0.913, respectivamente. Para tal estudo, a variável $\gamma$ indicou o pulso anormal nas artérias tibiais posteriores, sendo $T$ a idade de aparecimento da doença arterial periférica e $C$ a idade que o paciente tinha quando o teste foi realizado.

Calculamos a estimativa $\hat{F}_{\gamma}$ e $\hat{F}_{\text {cor }}$ da função de distribuição $F$ de T. A Figura 4.8 mostra o ENPMV $\hat{F}_{\gamma}$ e a estimativa corrigida $\hat{F}_{\text {cor }}$ de F. Para estes dados a função $\hat{F}_{c o r}$ parece indicar um risco desprezível de ocorrência de doença arterial periférica para idades abaixo de 65 anos, assim como visto também para as funções suavizadas.

Além disso, o ENPMV $\hat{F}_{\gamma}$ indica uma probabilidade de aproximadamente $4 \%$ de ocorrência da doença arterial para o paciente com idade de 45 anos, aproximada- 
mente.

De modo geral, a estimativa corrigida para a função de distribuição do aparecimento da doença arterial $\hat{F}_{\text {cor }}$ permitiu identificar que $\hat{F}_{\text {cor }}$ fornece probabilidades menores de ocorrência da doença arterial periférica que o ENPMV $\hat{F}_{\gamma}$ para idades abaixo de 75 anos, aproximadamente.

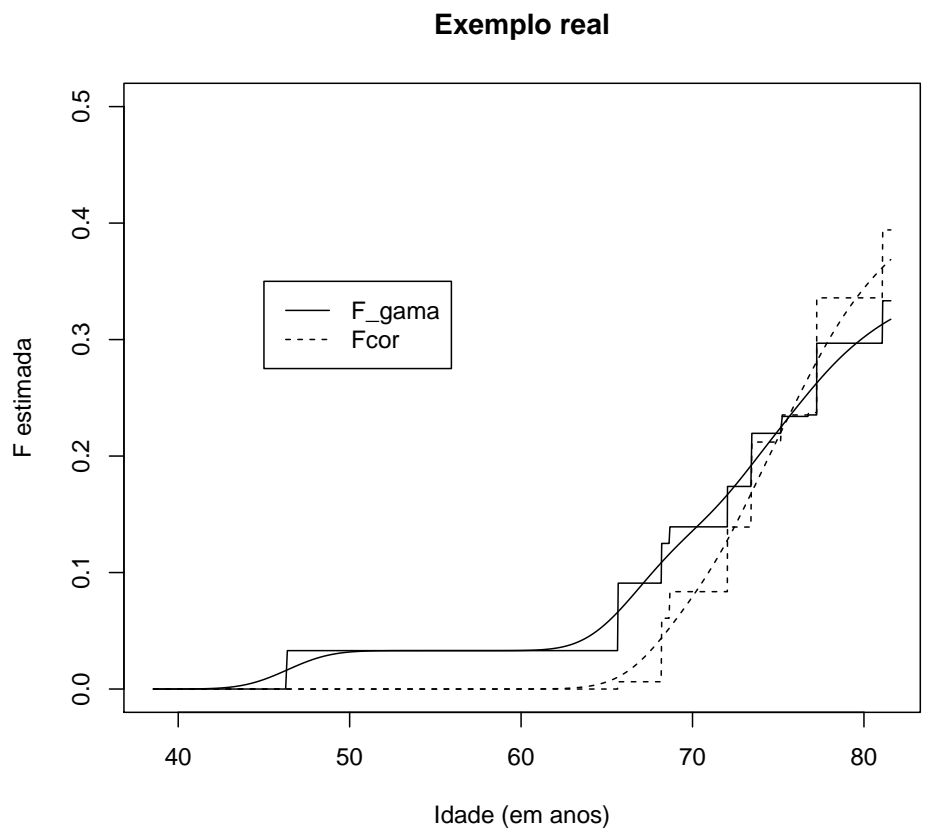

Figura 4.8: Gráfico da função suavizada de distribuição estimada do tempo de ocorrência da doença arterial 


\section{Conclusões e Trabalhos Futuros}

\subsection{Conclusões}

Em ambos os artigos (Gomes e da-Silva (2011) e Mckeown e Jewell (2010)), há a apresentação de um método não paramétrico para estimação da distribuição do tempo de falha F, levando em consideração os erros de classificação (o efeito da sensibilidade e da especificidade do teste da variável em estudo) e consequentemente no cálculo do ENPMV da F.

Através da comparação das várias estimativas da função procurada, podese identificar a diminuição do vício nos estimadores através do método de correção proposto.

Para $\hat{F}_{\delta}$, que é a estimativa com o $\delta$, caso em que não há erro de classificação (situação real difícil de ocorrer), sua estimativa foi muito próxima da curva real.

Para $\hat{F}_{\gamma}$, situação em que há vício forte nas caudas (com erro de classificação, sem correção), percebe-se uma estimativa bastante distante da curva real.

Para $\hat{F}_{c}$, situação obtida através dos métodos de correção para os erros de classificação, seu gráfico escada aparece muito próximo daquele que seria a curva obtida caso não houvesse erros de classificação $\left(\hat{F}_{\delta}\right)$ ou bem próximo da curva real $\mathrm{F}$. O método de correção reduz significativamente o vício do estimador não paramétrico de $\mathrm{F}$ na presença de erros de classificação em dados de status corrente. 


\subsection{Trabalhos Futuros}

O que se pretende para esta próxima etapa do trabalho é estudar métodos de estimação paramétrica para a distribuição $\mathrm{F}$ de $\mathrm{T}$ sob a presença de possíveis erros de classificação na determinação dos valores das variáveis indicadoras.

Desta forma, a sugestão é a utilização de um método de correção para o caso paramétrico, avaliando o vício e a variância dos estimadores corrigidos.

Um ponto teórico a ser explorado é a obtenção da distribuição assintótica do ENPMV de F na presença de erros de classificação e também do estimador corrigido.

Outra possibilidade é o estudo da estimação não paramétrica da distribuição do tempo de falha na presença de censura intervalar com erros de classificação.

Podemos ainda estudar o efeito da correção no ajuste dos modelos de regressão para o caso em que há a presença de covariáveis.

Outra sugestão de estudo é a utilização de um modelo de delineamento de experimentos para a verificação dos fatores que mais influenciam na diminuição do vício do ENPMV. Pode-se criar fatores que levem em consideração o tamanho da amostra, o cenário de censura, os níveis de sensibilidade e especificidade, a presença de suavização. Com isso, pode-se quantificar o vício estimado (variável resposta) para os diferentes conjuntos de fatores. 


\section{Referências Bibliográficas}

[1] AKAIKE, H., 1954. "An Approximation to the Density Function." Ann. Inst. Statistics. Math. 6, 127-32.

[2] AYER, M., BRUNK, H.D., EWING, G.M, REID, W.T., SILVERMAN, E., 1955. "An Empirical Distribution Function for Sampling with Incomplete Information." Ann. Math. Statist. 26, 641-647

[3] BARLOW, R.E., BARTHOLOMEW, D.J., BREMMER, J.M. e BRUNK, H.D., 1972. "Statistical Inference Under Order Restrictions." John Wiley \& Sons, New York.

[4] BASU, D., 1977. "On the elimination of nuisance parameters." J. Amer. Statist. Assoc. 72, 355-366.

[5] BERGER,J. O.; LISEO, B.; WOLPERT, R. L., 1999 . "Integrated likelihood methods for eliminating nuisance parameters" Statistical Science, 1999, Vol. 14, No. 1, 1-28.

[6] BERGER,J. O . and WOLPERT, R. L., 1988. "The Likelihood Principle: A Review, Generalizations, and Statistical Implications" 2nd ed. IMS, Hayward, $C A$.

[7] BJORNSTAD, J., 1996. "On the generalization of the likelihood function and the likelihood principle". J Amer. Statist. Assoc. 91, 791-806.

[8] CRIQUi, M. H.; FRONEK, A.; KLAUBER, M. R.; BARRETT-CONNOR, E.; GABRIEL, S. "The sensitivity, specificity, and predictive value of traditional clinical evaluation of peripheral arterial disease: results from noninvasive testing in a defined population". Circulation, Dallas, v.71, p.516-522, 1985. 
[9] COX, D. R. (1975). "Partial likelihood". Biometrika 62 269-276.

[10] DOUGLAS, J; COHEN, A. (2001). "Nonparametric item response function estimation for assessing parametric model fit." Applied Psychological Measurement, 25, pp. 234-243.

[11] EPANECHNIKOV, V. A. (1969). "Non-parametric estimation of a multivariate probability density". Theory of Probability and its Applications 14, 153-158.

[12] FRASER, D. A. S; REID N. (1989). "Adjustments to profile likelihood" Biometrika (1989), 76, 3, pp. 447-88.

[13] GONÇALVES, R. P., 2011. "Ajuste de modelos de platô de resposta via regressão isotônica" Dissertação de Doutorado, Lavras - $M G$.

[14] GOMES, A. E.; da-SILVA, C. Q. "Nonparametric maximum likelihood estimation for current status data with misclassification." Revista Brasileira de Biometria, v. 29, p. 102-121, 2011.

[15] GROENEBOOM, P.; WELLNER, J.A. (1992) "Information Bounds and Nonparametric Maximum Likelihood Estimation". Birkhauser Verlag, Berlin.

[16] JONGBLOED, G. (1999). "Inverse problems in statistics". Vrije University

[17] KALBFLEISCH, D. A. (1970). "Application of like- J.D. and SPROTT, lihood methods to models involving large numbers of parameters". J. Roy. Statist. Soc. Ser B 32 175-208.

[18] LEE, E. T. 1992. "Statistical Methods for Survival Data Analysis." John Wiley 83 Sons, New York.

[19] LOPES, E. R. 2007. "Intervalos de confiança para a função de distribuição na presença de censura intervalar, caso 1". Dissertação de Mestrado, Belo Horizonte - $M G$.

[20] MCKEOWN, K.; JEWELL, N. P. "Misclassification of current status data". Lifetime Data Anal., Boston, v.16, n.2, p.215-230, 2010. 
[21] ROBERTSON, T., F. WRIGHT, and R. DYKSTRA (1988). "Order Restricted Statistical Inference.". John Wiley and Sons.

[22] RODRIGUES, A. 2010. "Utilização da regressão isotônica em estudos de curva de crescimento". Dissertação de Doutorado, Lavras - MG.

[23] ROSENBLATT, M. (1956). "Remarks on some nonparametrics estimates of a density function". The Annals of Mathematical Statistics. 27, 832-837.

[24] SCOTT, D. W. (1992). "Multivariate Density Estimation: Theory, Practice, and Visualization". New York: John Wiley 6 Sons.

[25] SILVERMAN, B. W. (1986). "Density Estimation for Statistics and Data Analysis". London: Chapman \& Hall.

[26] SIMONOFF, J. S. (1996). "Smoothing Methods in Statistics". New York: Springer-Verlag.

[27] STRAPASSON, E. 2007. "Comparação de modelos com censura intervalar em análise de sobrevivência". Dissertação de Doutorado, Piracicaba.

[28] SUN, J. 1998. "Interval Censoring". Encyclopedia of Biostatistics. First Edition, John Wiley 6 Sons, New York. 


\section{Apêndice 1}

O Teorema 1.10 (em Barlow et al., 1972 - Função de máximo/ mínimo) mostra que:

Se f é isotônica em X e se o domínio de f está em I, então:

$$
\begin{gathered}
\sum_{x} \Delta[g(x), f(x)] w(x) \geq \\
\sum_{x} \Delta\left[g(x), g^{*}(x)\right] w(x)+\sum_{x} \Delta\left[g^{*}(x), f(x)\right] w(x)
\end{gathered}
$$

Consequentemente $g^{*}$ minimiza

$$
\sum_{x} \Delta[g(x), f(x)] w(x)
$$

na classe das isotônicas f com domínio em I e máximiza

$$
\sum_{x}\{\Phi[f(x)]+[g(x)-f(x)] \varphi[f(x)]\} w(x)
$$

A função de máximo (mínimo) é única se $\Phi$ é extritamente convexo.

\section{Corolário}

Seja $\psi_{1}, \psi_{2}, \ldots, \psi_{p}$ serem valores de funções reais arbitrárias e seja $h_{1}, h_{2}, \ldots, h_{m}$ serem funções isotônicas em X.

Então, $g^{*}$ minimiza

$$
\sum_{x} \Delta[g(x), f(x)] w(x)
$$

na classe de funções isotônicas f com domínio em I satisfazendo alguma condição ou todos os lados das condições

$$
\begin{gathered}
\sum_{x}[g(x), f(x)] \varphi_{i}[f(x)] w(x)=0, \quad j=1,2, \ldots p \\
\sum_{x} f(x) h_{j}(x) w(x) \geq \sum g(x) h_{j}(x) w(x), \quad j=1,2, \ldots p
\end{gathered}
$$


Tabela 4.1: Estimação da esperança e erro-padrão de $F_{\gamma}, F_{\delta}$ e $F_{c o r}$ no quantil da T, SEM suavização, com $50 \%$ de censura à esquerda e $50 \%$ à direita

\begin{tabular}{|c|c|c|c|c|c|c|c|}
\hline \multirow[b]{2}{*}{$\mathrm{n}$} & \multirow[b]{2}{*}{$\mathrm{s}$} & \multirow[b]{2}{*}{$\mathrm{e}$} & & \multicolumn{2}{|l|}{ Quantil } & \multirow[b]{2}{*}{0.5} & \multirow[b]{2}{*}{0.75} \\
\hline & & & & 0.10 & 0.25 & & \\
\hline \multirow[t]{6}{*}{200} & 0.7 & 0.7 & $\hat{F}_{\gamma}$ & 0.31453190 & 0.39135880 & 0.49881340 & 0.60227930 \\
\hline & & & $\hat{\sigma}_{\gamma}$ & $(0.314531)$ & $(0.391358)$ & $(0.498813)$ & $(0.602279)$ \\
\hline & & & $\hat{F}_{\delta}$ & 0.06962473 & 0.23242778 & 0.49989409 & 0.75773028 \\
\hline & & & $\hat{\sigma}_{\delta}$ & $(0.067797)$ & $(0.084262)$ & $(0.087688)$ & $(0.084663)$ \\
\hline & & & $\hat{F}_{\text {cor }}$ & 0.10138660 & 0.23556880 & 0.49719050 & 0.74998930 \\
\hline & & & $\hat{\sigma}_{c o r}$ & $(0.126497)$ & $(0.161301)$ & $(0.165686)$ & $(0.155449)$ \\
\hline \multirow[t]{6}{*}{200} & 0.9 & 0.7 & $\hat{F}_{\gamma}$ & 0.33938310 & 0.44476390 & 0.60143110 & 0.75592020 \\
\hline & & & $\hat{\sigma}_{\gamma}$ & $(0.090445)$ & $(0.078463)$ & $(0.073333)$ & $(0.070339)$ \\
\hline & & & $\hat{F}_{\delta}$ & 0.06962473 & 0.23242778 & 0.49989409 & 0.75773028 \\
\hline & & & $\hat{\sigma}_{\delta}$ & $(0.067797)$ & $(0.084262)$ & $(0.087688)$ & $(0.084663)$ \\
\hline & & & $\hat{F}_{c o r}$ & 0.09907556 & 0.24266940 & 0.50238523 & 0.75928267 \\
\hline & & & $\hat{\sigma}_{c o r}$ & $(0.105105)$ & $(0.127757)$ & $(0.122222)$ & $(0.115885)$ \\
\hline \multirow[t]{6}{*}{200} & 0.7 & 0.9 & $\hat{F}_{\gamma}$ & 0.13668150 & 0.23819620 & 0.39797370 & 0.54952580 \\
\hline & & & $\hat{\sigma}_{\gamma}$ & $(0.072769)$ & $(0.069950)$ & $(0.073910)$ & $(0.075517)$ \\
\hline & & & $\hat{F}_{\delta}$ & 0.06962473 & 0.23242778 & 0.49989409 & 0.75773028 \\
\hline & & & $\hat{\sigma}_{\delta}$ & $(0.067797)$ & $(0.084262)$ & $(0.087688)$ & $(0.084663)$ \\
\hline & & & $\hat{F}_{\text {cor }}$ & 0.08489135 & 0.23094749 & 0.49662279 & 0.74781940 \\
\hline & & & $\hat{\sigma}_{c o r}$ & $(0.091754)$ & $(0.115169)$ & $(0.123184)$ & $(0.122444)$ \\
\hline \multirow[t]{6}{*}{200} & 0.9 & 0.9 & $\hat{F}_{\gamma}$ & 0.15760700 & 0.28801230 & 0.50008450 & 0.70436380 \\
\hline & & & $\hat{\sigma}_{\gamma}$ & $(0.081826)$ & $(0.080205)$ & $(0.082775)$ & $(0.080698)$ \\
\hline & & & $\hat{F}_{\delta}$ & 0.06962473 & 0.23242778 & 0.49989409 & 0.75773028 \\
\hline & & & $\hat{\sigma}_{\delta}$ & $(0.067797)$ & $(0.084262)$ & $(0.087688)$ & $(0.084663)$ \\
\hline & & & $\hat{F}_{c o r}$ & 0.08628240 & 0.23510320 & 0.50010560 & 0.75533300 \\
\hline & & & $\hat{\sigma}_{c o r}$ & $(0.083085)$ & $(0.100039)$ & $(0.103469)$ & $(0.100541)$ \\
\hline
\end{tabular}


Tabela 4.2: Estimação da esperança e erro-padrão de $F_{\gamma}, F_{\delta}$ e $F_{c o r}$ no quantil da T, SEM suavização, com 50\% de censura à esquerda e 50\% à direita

\begin{tabular}{|c|c|c|c|c|c|c|c|}
\hline \multirow[b]{2}{*}{$\mathrm{n}$} & \multirow[b]{2}{*}{$\mathrm{S}$} & \multirow[b]{2}{*}{ e } & & \multicolumn{2}{|l|}{ Quantil } & \multirow[b]{2}{*}{0.5} & \multirow[b]{2}{*}{0.75} \\
\hline & & & & 0.10 & 0.25 & & \\
\hline \multirow[t]{6}{*}{500} & 0.7 & 0.7 & $\hat{F}_{\gamma}$ & 0.32741370 & 0.39881760 & 0.49770690 & 0.60103410 \\
\hline & & & $\hat{\sigma}_{\gamma}$ & $(0.054854)$ & $(0.049794)$ & $(0.0481784)$ & $(0.048978)$ \\
\hline & & & $\hat{F}_{\delta}$ & 0.08629490 & 0.24263810 & 0.49968750 & 0.75483310 \\
\hline & & & $\hat{\sigma}_{\delta}$ & $(0.050742)$ & $(0.059033)$ & $(0.0647342)$ & $(0.058427)$ \\
\hline & & & $\hat{F}_{c o r}$ & 0.09587421 & 0.24797065 & 0.49426728 & 0.75194892 \\
\hline & & & $\hat{\sigma}_{c o r}$ & $(0.101557)$ & $(0.122323)$ & $(0.1204460)$ & $(0.120846)$ \\
\hline \multirow[t]{6}{*}{500} & 0.9 & 0.7 & $\hat{F}_{\gamma}$ & 0.34955970 & 0.44990690 & 0.59922070 & 0.75339090 \\
\hline & & & $\hat{\sigma}_{\gamma}$ & $(0.059684)$ & $(0.055498)$ & $(0.0548601)$ & $(0.050889)$ \\
\hline & & & $\hat{F}_{\delta}$ & 0.08629490 & 0.24263810 & 0.49968750 & 0.75483310 \\
\hline & & & $\hat{\sigma}_{\delta}$ & $(0.050742)$ & $(0.059033)$ & $(0.0647342)$ & $(0.058427)$ \\
\hline & & & $\hat{F}_{\text {cor }}$ & 0.09435393 & 0.24989358 & 0.49870124 & 0.75564654 \\
\hline & & & $\hat{\sigma}_{c o r}$ & $(0.082273)$ & $(0.092359)$ & $(0.0914336)$ & $(0.084802)$ \\
\hline \multirow[t]{6}{*}{500} & 0.7 & 0.9 & $\hat{F}_{\gamma}$ & 0.15154170 & 0.24404070 & 0.39755240 & 0.55102540 \\
\hline & & & $\hat{\sigma}_{\gamma}$ & $(0.047747)$ & $(0.050035)$ & $(0.0542672)$ & $(0.055843)$ \\
\hline & & & $\hat{F}_{\delta}$ & 0.08629490 & 0.24263810 & 0.49968750 & 0.75483310 \\
\hline & & & $\hat{\sigma}_{\delta}$ & $(0.050742)$ & $(0.059033)$ & $(0.0647342)$ & $(0.058427)$ \\
\hline & & & $\hat{F}_{c o r}$ & 0.09117618 & 0.24006784 & 0.49592058 & 0.75156849 \\
\hline & & & $\hat{\sigma}_{c o r}$ & $(0.071311)$ & $(0.083392)$ & $(0.0904453)$ & $(0.09265114$ \\
\hline \multirow[t]{6}{*}{500} & 0.9 & 0.9 & $\hat{F}_{\gamma}$ & 0.17179920 & 0.29472460 & 0.49900120 & 0.70388970 \\
\hline & & & $\hat{\sigma}_{\gamma}$ & $(0.052581)$ & $(0.056674)$ & $(0.0601302)$ & $(0.056910)$ \\
\hline & & & $\hat{F}_{\delta}$ & 0.08629490 & 0.24263810 & 0.49968750 & 0.75483310 \\
\hline & & & $\hat{\sigma}_{\delta}$ & $(0.050742)$ & $(0.059033)$ & $(0.0647342)$ & $(0.058427)$ \\
\hline & & & $\hat{F}_{c o r}$ & 0.09212276 & 0.24340574 & 0.49875156 & 0.75486216 \\
\hline & & & $\hat{\sigma}_{c o r}$ & $(0.061423)$ & $(0.070843)$ & $(0.0751628)$ & $(0.071137)$ \\
\hline
\end{tabular}


Tabela 4.3: Estimação da esperança e erro-padrão de $F_{\gamma}, F_{\delta}$ e $F_{c o r}$ no quantil da T, SEM suavização, com $50 \%$ de censura à esquerda e $50 \%$ à direita

\begin{tabular}{|c|c|c|c|c|c|c|c|}
\hline \multirow[b]{2}{*}{$\mathrm{n}$} & \multirow[b]{2}{*}{$\mathrm{S}$} & \multirow[b]{2}{*}{ e } & & \multicolumn{2}{|l|}{ Quantil } & \multirow[b]{2}{*}{0.5} & \multirow[b]{2}{*}{0.75} \\
\hline & & & & 0.10 & 0.25 & & \\
\hline \multirow[t]{6}{*}{1000} & 0.7 & 0.7 & $\hat{F}_{\gamma}$ & 0.33316250 & 0.39726830 & 0.49891420 & 0.60184510 \\
\hline & & & $\hat{\sigma}_{\gamma}$ & $(0.039969)$ & $(0.037298)$ & $(0.038734)$ & $(0.039327)$ \\
\hline & & & $\hat{F}_{\delta}$ & 0.08861313 & 0.24392488 & 0.49729059 & 0.75441700 \\
\hline & & & $\hat{\sigma}_{\delta}$ & $(0.036859)$ & $(0.047485)$ & $(0.053567)$ & $(0.048636)$ \\
\hline & & & $\hat{F}_{c o r}$ & 0.09464439 & 0.24317646 & 0.49728539 & 0.75446550 \\
\hline & & & $\hat{\sigma}_{c o r}$ & $(0.081430)$ & $(0.093230)$ & $(0.096837)$ & $(0.097902)$ \\
\hline \multirow[t]{6}{*}{1000} & 0.9 & 0.7 & $\hat{F}_{\gamma}$ & 0.35485410 & 0.44715880 & 0.59980700 & 0.75209630 \\
\hline & & & $\hat{\sigma}_{\gamma}$ & $(0.044820)$ & $(0.043302)$ & $(0.044183)$ & $(0.040266)$ \\
\hline & & & $\hat{F}_{\delta}$ & 0.08861313 & 0.24392488 & 0.49729059 & 0.75441700 \\
\hline & & & $\hat{\sigma}_{\delta}$ & $(0.036859)$ & $(0.047485)$ & $(0.053567)$ & $(0.048636)$ \\
\hline & & & $\hat{F}_{c o r}$ & 0.09556714 & 0.24526465 & 0.49967831 & 0.75349389 \\
\hline & & & $\hat{\sigma}_{c o r}$ & $(0.067156)$ & $(0.072171)$ & $(0.073638)$ & $(0.067111)$ \\
\hline \multirow[t]{6}{*}{1000} & 0.7 & 0.9 & $\hat{F}_{\gamma}$ & 0.15421100 & 0.24444330 & 0.39666320 & 0.55311180 \\
\hline & & & $\hat{\sigma}_{\gamma}$ & $(0.036703)$ & $(0.040266)$ & $(0.043816)$ & $(0.045763)$ \\
\hline & & & $\hat{F}_{\delta}$ & 0.08861313 & 0.24392488 & 0.49729059 & 0.75441700 \\
\hline & & & $\hat{\sigma}_{\delta}$ & $(0.036859)$ & $(0.047485)$ & $(0.053567)$ & $(0.048636)$ \\
\hline & & & $\hat{F}_{c o r}$ & 0.09202400 & 0.24073880 & 0.49443860 & 0.75518630 \\
\hline & & & $\hat{\sigma}_{c o r}$ & $(0.057991)$ & $(0.067111)$ & $(0.073028)$ & $(0.076272)$ \\
\hline \multirow[t]{6}{*}{1000} & 0.9 & 0.9 & $\hat{F}_{\gamma}$ & 0.17448610 & 0.29482090 & 0.49681910 & 0.70368480 \\
\hline & & & $\hat{\sigma}_{\gamma}$ & $(0.041477)$ & $(0.046020)$ & $(0.049461)$ & $(0.046003)$ \\
\hline & & & $\hat{F}_{\delta}$ & 0.08861313 & 0.24392488 & 0.49729059 & 0.75441700 \\
\hline & & & $\hat{\sigma}_{\delta}$ & $(0.036859)$ & $(0.047485)$ & $(0.053567)$ & $(0.048636)$ \\
\hline & & & $\hat{F}_{c o r}$ & 0.09371824 & 0.24352607 & 0.49602388 & 0.75460602 \\
\hline & & & $\hat{\sigma}_{c o r}$ & $(0.050527)$ & $(0.057525)$ & $(0.061826)$ & $(0.057504)$ \\
\hline
\end{tabular}


Tabela 4.4: Estimação da esperança e erro-padrão de $F_{\gamma}, F_{\delta}$ e $F_{c o r}$ no quantil da T, COM suavização, com 50\% de censura à esquerda e 50\% à direita

\begin{tabular}{|c|c|c|c|c|c|c|c|}
\hline \multirow[b]{2}{*}{$\mathrm{n}$} & \multirow[b]{2}{*}{$\mathrm{s}$} & \multirow[b]{2}{*}{ e } & & \multicolumn{2}{|l|}{ Quantil } & \multirow[b]{2}{*}{0.5} & \multirow[b]{2}{*}{0.75} \\
\hline & & & & 0.10 & 0.25 & & \\
\hline \multirow[t]{6}{*}{200} & 0.7 & 0.7 & $\hat{F}_{\gamma}$ & 0.28357070 & 0.38744430 & 0.49522700 & 0.59820900 \\
\hline & & & $\hat{\sigma}_{\gamma}$ & $(0.054340)$ & $(0.047595)$ & $(0.044028)$ & $(0.049001)$ \\
\hline & & & $\hat{F}_{\delta}$ & 0.10406230 & 0.24651550 & 0.49130500 & 0.74390450 \\
\hline & & & $\hat{\sigma}_{\delta}$ & $(0.030618)$ & $(0.040868)$ & $(0.046091)$ & $(0.047934)$ \\
\hline & & & $\hat{F}_{\text {cor }}$ & 0.12183660 & 0.25481000 & 0.48948420 & 0.73206280 \\
\hline & & & $\hat{\sigma}_{c o r}$ & $(0.083529)$ & $(0.104009)$ & $(0.108788)$ & $(0.110265)$ \\
\hline \multirow[t]{6}{*}{200} & 0.9 & 0.7 & $\hat{F}_{\gamma}$ & 0.31141170 & 0.44287220 & 0.59757490 & 0.74761900 \\
\hline & & & $\hat{\sigma}_{\gamma}$ & $(0.056677)$ & $(0.049082)$ & $(0.043750)$ & $(0.046193)$ \\
\hline & & & $\hat{F}_{\delta}$ & 0.10406230 & 0.24651550 & 0.49130500 & 0.74390450 \\
\hline & & & $\hat{\sigma}_{\delta}$ & $(0.030618)$ & $(0.040868)$ & $(0.046091)$ & $(0.047934)$ \\
\hline & & & $\hat{F}_{\text {cor }}$ & 0.12138330 & 0.25800210 & 0.49659090 & 0.74248600 \\
\hline & & & $\hat{\sigma}_{c o r}$ & $(0.064123)$ & $(0.075214)$ & $(0.072804)$ & $(0.074917)$ \\
\hline \multirow[t]{6}{*}{200} & 0.7 & 0.9 & $\hat{F}_{\gamma}$ & 0.13851430 & 0.24209610 & 0.39179210 & 0.54204320 \\
\hline & & & $\hat{\sigma}_{\gamma}$ & $(0.040141)$ & $(0.041985)$ & $(0.043564)$ & $(0.051502)$ \\
\hline & & & $\hat{F}_{\delta}$ & 0.10406230 & 0.24651550 & 0.49130500 & 0.74390450 \\
\hline & & & $\hat{\sigma}_{\delta}$ & $(0.030618)$ & $(0.040868)$ & $(0.046091)$ & $(0.047934)$ \\
\hline & & & $\hat{F}_{c o r}$ & 0.11280510 & 0.24802160 & 0.48654010 & 0.73116590 \\
\hline & & & $\hat{\sigma}_{c o r}$ & $(0.053821)$ & $(0.066741)$ & $(0.072444)$ & $(0.081498)$ \\
\hline \multirow[t]{6}{*}{200} & 0.9 & 0.9 & $\hat{F}_{\gamma}$ & 0.16365640 & 0.29562100 & 0.49379040 & 0.69302900 \\
\hline & & & $\hat{\sigma}_{\gamma}$ & $(0.042833)$ & $(0.045064)$ & $(0.045500)$ & $(0.050441)$ \\
\hline & & & $\hat{F}_{\delta}$ & 0.10406230 & 0.24651550 & 0.49130500 & 0.74390450 \\
\hline & & & $\hat{\sigma}_{\delta}$ & $(0.030618)$ & $(0.040868)$ & $(0.046091)$ & $(0.047934)$ \\
\hline & & & $\hat{F}_{c o r}$ & 0.11432090 & 0.25193460 & 0.49244030 & 0.73950890 \\
\hline & & & $\hat{\sigma}_{c o r}$ & $(0.045186)$ & $(0.054637)$ & $(0.056856)$ & $(0.062279)$ \\
\hline
\end{tabular}


Tabela 4.5: Estimação da esperança e erro-padrão de $F_{\gamma}, F_{\delta}$ e $F_{c o r}$ no quantil da T, COM suavização, com 50\% de censura à esquerda e 50\% à direita

\begin{tabular}{|c|c|c|c|c|c|c|c|}
\hline \multirow[b]{2}{*}{$\mathrm{n}$} & \multirow[b]{2}{*}{$\mathrm{s}$} & \multirow[b]{2}{*}{ e } & & \multicolumn{2}{|l|}{ Quantil } & \multirow[b]{2}{*}{0.5} & \multirow[b]{2}{*}{0.75} \\
\hline & & & & 0.10 & 0.25 & & \\
\hline \multirow[t]{6}{*}{500} & 0.7 & 0.7 & $\hat{F}_{\gamma}$ & 0.31216400 & 0.39825340 & 0.49626230 & 0.59744610 \\
\hline & & & $\hat{\sigma}_{\gamma}$ & $(0.037641)$ & $(0.032395)$ & $(0.030285)$ & $(0.034858)$ \\
\hline & & & $\hat{F}_{\delta}$ & 0.10473550 & 0.25062690 & 0.49419320 & 0.74534920 \\
\hline & & & $\hat{\sigma}_{\delta}$ & $(0.022107)$ & $(0.028263)$ & $(0.031667)$ & $(0.032714)$ \\
\hline & & & $\hat{F}_{\text {cor }}$ & 0.11734780 & 0.25591110 & 0.49076540 & 0.74043800 \\
\hline & & & $\hat{\sigma}_{c o r}$ & $(0.065980)$ & $(0.077392)$ & $(0.075643)$ & $(0.084819)$ \\
\hline \multirow[t]{6}{*}{500} & 0.9 & 0.7 & $\hat{F}_{\gamma}$ & 0.33801360 & 0.45147690 & 0.59633460 & 0.74690430 \\
\hline & & & $\hat{\sigma}_{\gamma}$ & $(0.038913)$ & $(0.033640)$ & $(0.030824)$ & $(0.032616)$ \\
\hline & & & $\hat{F}_{\delta}$ & 0.10473550 & 0.25062690 & 0.49419320 & 0.74534920 \\
\hline & & & $\hat{\sigma}_{\delta}$ & $(0.022107)$ & $(0.028263)$ & $(0.031667)$ & $(0.032714)$ \\
\hline & & & $\hat{F}_{c o r}$ & 0.11672940 & 0.25758520 & 0.49393520 & 0.74435470 \\
\hline & & & $\hat{\sigma}_{c o r}$ & $(0.049461)$ & $(0.054743)$ & $(0.051368)$ & $(0.054136)$ \\
\hline \multirow[t]{6}{*}{500} & 0.7 & 0.9 & $\hat{F}_{\gamma}$ & 0.15024910 & 0.24857850 & 0.39399690 & 0.54469500 \\
\hline & & & $\hat{\sigma}_{\gamma}$ & $(0.028654)$ & $(0.028405)$ & $(0.030070)$ & $(0.036716)$ \\
\hline & & & $\hat{F}_{\delta}$ & 0.10473550 & 0.25062690 & 0.49419320 & 0.74534920 \\
\hline & & & $\hat{\sigma}_{\delta}$ & $(0.022107)$ & $(0.028263)$ & $(0.031667)$ & $(0.032714)$ \\
\hline & & & $\hat{F}_{c o r}$ & 0.11052880 & 0.25041260 & 0.49000310 & 0.73998680 \\
\hline & & & $\hat{\sigma}_{c o r}$ & $(0.039909)$ & $(0.046792)$ & $(0.050115)$ & $(0.060410)$ \\
\hline \multirow[t]{6}{*}{500} & 0.9 & 0.9 & $\hat{F}_{\gamma}$ & 0.17407830 & 0.30080230 & 0.49431960 & 0.69482150 \\
\hline & & & $\hat{\sigma}_{\gamma}$ & $(0.030273)$ & $(0.030207)$ & $(0.031398)$ & $(0.034892)$ \\
\hline & & & $\hat{F}_{\delta}$ & 0.10473550 & 0.25062690 & 0.49419320 & 0.74534920 \\
\hline & & & $\hat{\sigma}_{\delta}$ & $(0.022107)$ & $(0.028263)$ & $(0.031667)$ & $(0.032714)$ \\
\hline & & & $\hat{F}_{c o r}$ & 0.11138320 & 0.25274540 & 0.49290770 & 0.74331440 \\
\hline & & & $\hat{\sigma}_{c o r}$ & $(0.032724)$ & $(0.037439)$ & $(0.039247)$ & $(0.043553)$ \\
\hline
\end{tabular}


Tabela 4.6: Estimação da esperança e erro-padrão de $F_{\gamma}, F_{\delta}$ e $F_{c o r}$ no quantil da T, COM suavização, com 50\% de censura à esquerda e 50\% à direita

\begin{tabular}{|c|c|c|c|c|c|c|c|}
\hline \multirow[b]{2}{*}{$\mathrm{n}$} & \multirow[b]{2}{*}{$\mathrm{s}$} & \multirow[b]{2}{*}{ e } & & \multicolumn{2}{|l|}{ Quantil } & \multirow[b]{2}{*}{0.5} & \multirow[b]{2}{*}{0.75} \\
\hline & & & & 0.10 & 0.25 & & \\
\hline \multirow[t]{6}{*}{1000} & 0.7 & 0.7 & $\hat{F}_{\gamma}$ & 0.32551550 & 0.39925490 & 0.49775780 & 0.59755780 \\
\hline & & & $\hat{\sigma}_{\gamma}$ & $(0.028579)$ & $(0.023884)$ & $(0.024329)$ & $(0.026871)$ \\
\hline & & & $\hat{F}_{\delta}$ & 0.10340710 & 0.25051590 & 0.49486210 & 0.74448660 \\
\hline & & & $\hat{\sigma}_{\delta}$ & $(0.016933)$ & $(0.021601)$ & $(0.025062)$ & $(0.025877)$ \\
\hline & & & $\hat{F}_{\text {cor }}$ & 0.11112610 & 0.25127080 & 0.49440480 & 0.74304460 \\
\hline & & & $\hat{\sigma}_{c o r}$ & $(0.053879)$ & $(0.058205)$ & $(0.060816)$ & $(0.066419)$ \\
\hline \multirow[t]{6}{*}{1000} & 0.9 & 0.7 & $\hat{F}_{\gamma}$ & 0.35015010 & 0.45140900 & 0.59782900 & 0.74649450 \\
\hline & & & $\hat{\sigma}_{\gamma}$ & $(0.029744)$ & $(0.025146)$ & $(0.024415)$ & $(0.024820)$ \\
\hline & & & $\hat{F}_{\delta}$ & 0.10340710 & 0.25051590 & 0.49486210 & 0.74448660 \\
\hline & & & $\hat{\sigma}_{\delta}$ & $(0.016933)$ & $(0.021601)$ & $(0.025062)$ & $(0.025877)$ \\
\hline & & & $\hat{F}_{c o r}$ & 0.11209740 & 0.25370530 & 0.49638440 & 0.74411450 \\
\hline & & & $\hat{\sigma}_{c o r}$ & $(0.040459)$ & $(0.041426)$ & $(0.040691)$ & $(0.041360)$ \\
\hline \multirow[t]{6}{*}{1000} & 0.7 & 0.9 & $\hat{F}_{\gamma}$ & 0.15488980 & 0.24856290 & 0.39594280 & 0.54573860 \\
\hline & & & $\hat{\sigma}_{\gamma}$ & $(0.022523)$ & $(0.021337)$ & $(0.024643)$ & $(0.028657)$ \\
\hline & & & $\hat{F}_{\delta}$ & 0.10340710 & 0.25051590 & 0.49486210 & 0.74448660 \\
\hline & & & $\hat{\sigma}_{\delta}$ & $(0.016933)$ & $(0.021601)$ & $(0.025062)$ & $(0.025877)$ \\
\hline & & & $\hat{F}_{c o r}$ & 0.10635070 & 0.24832070 & 0.49323880 & 0.74273050 \\
\hline & & & $\hat{\sigma}_{c o r}$ & $(0.033215)$ & $(0.035450)$ & $(0.041071)$ & $(0.047691)$ \\
\hline \multirow[t]{6}{*}{1000} & 0.9 & 0.9 & $\hat{F}_{\gamma}$ & 0.17804060 & 0.30036940 & 0.49589670 & 0.69509250 \\
\hline & & & $\hat{\sigma}_{\gamma}$ & $(0.023737)$ & $(0.022922)$ & $(0.025252)$ & $(0.026768)$ \\
\hline & & & $\hat{F}_{\delta}$ & 0.10340710 & 0.25051590 & 0.49486210 & 0.74448660 \\
\hline & & & $\hat{\sigma}_{\delta}$ & $(0.016933)$ & $(0.021601)$ & $(0.025062)$ & $(0.025877)$ \\
\hline & & & $\hat{F}_{c o r}$ & 0.10792610 & 0.25089340 & 0.49487120 & 0.74385360 \\
\hline & & & $\hat{\sigma}_{c o r}$ & $(0.027007)$ & $(0.028607)$ & $(0.031565)$ & $(0.033459)$ \\
\hline
\end{tabular}


Tabela 4.7: Estimação da esperança e erro-padrão de $F_{\gamma}, F_{\delta}$ e $F_{c o r}$ no quantil da T, SEM suavização, com $70 \%$ de censura à esquerda e 30\% à direita

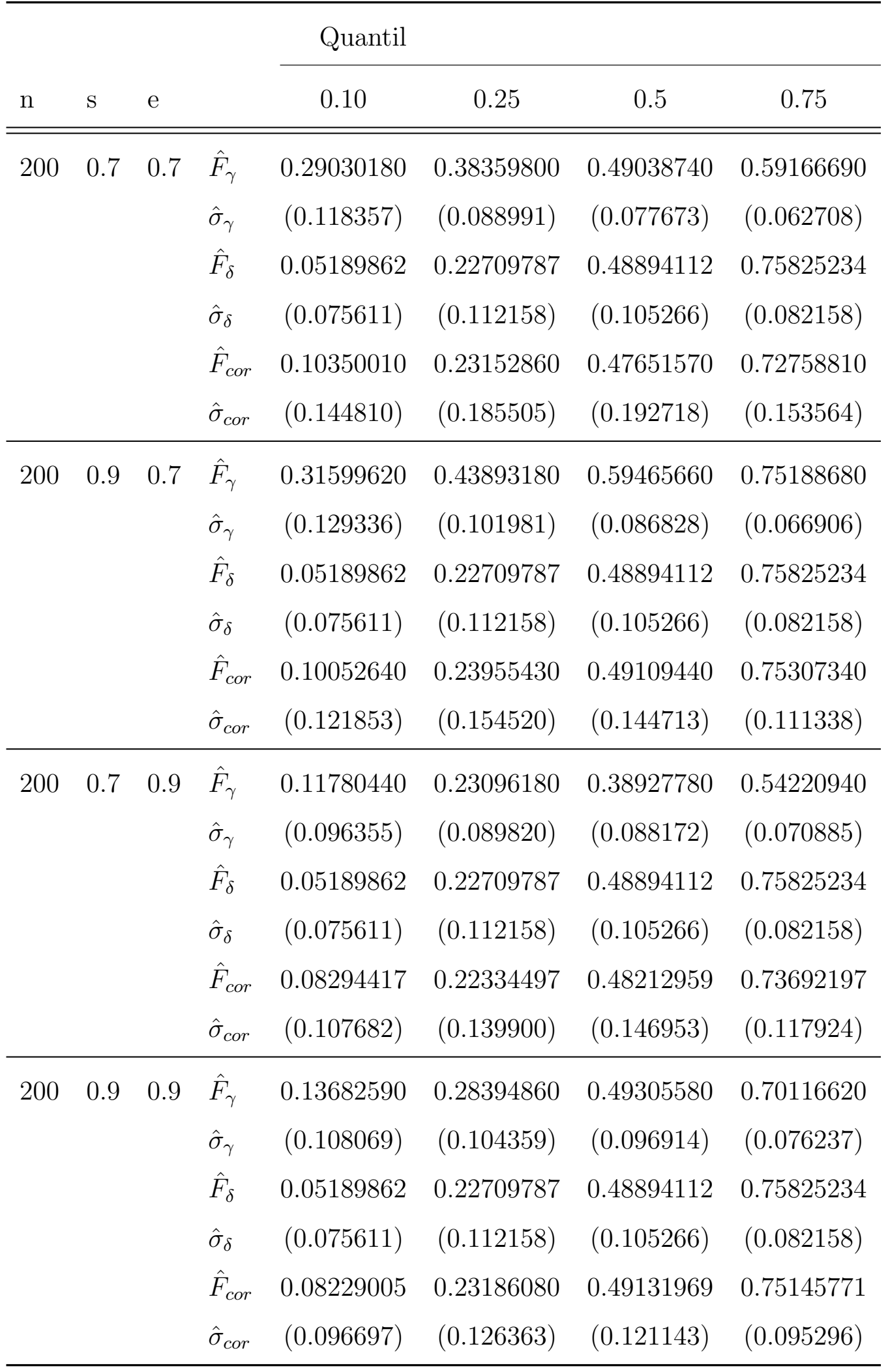


Tabela 4.8: Estimação da esperança e erro-padrão de $F_{\gamma}, F_{\delta}$ e $F_{c o r}$ no quantil da T, SEM suavização, com $70 \%$ de censura à esquerda e 30\% à direita

\begin{tabular}{|c|c|c|c|c|c|c|c|}
\hline \multirow[b]{2}{*}{$\mathrm{n}$} & \multirow[b]{2}{*}{$\mathrm{s}$} & \multirow[b]{2}{*}{ e } & & \multicolumn{2}{|l|}{ Quantil } & \multirow[b]{2}{*}{0.5} & \multirow[b]{2}{*}{0.75} \\
\hline & & & & 0.10 & 0.25 & & \\
\hline \multirow[t]{6}{*}{500} & 0.7 & 0.7 & $\hat{F}_{\gamma}$ & 0.31792760 & 0.39647830 & 0.49430160 & 0.59423390 \\
\hline & & & $\hat{\sigma}_{\gamma}$ & $(0.076870)$ & $(0.062382)$ & $(0.053471)$ & $(0.048145)$ \\
\hline & & & $\hat{F}_{\delta}$ & 0.06799214 & 0.24448450 & 0.49317147 & 0.75309426 \\
\hline & & & $\hat{\sigma}_{\delta}$ & $(0.063831)$ & $(0.075284)$ & $(0.072030)$ & $(0.060368)$ \\
\hline & & & $\hat{F}_{c o r}$ & 0.10112540 & 0.24549970 & 0.48575390 & 0.73540930 \\
\hline & & & $\hat{\sigma}_{c o r}$ & $(0.123114)$ & $(0.147521)$ & $(0.133679)$ & $(0.119919)$ \\
\hline \multirow[t]{6}{*}{500} & 0.9 & 0.7 & $\hat{F}_{\gamma}$ & 0.34076860 & 0.45046990 & 0.59518980 & 0.74693640 \\
\hline & & & $\hat{\sigma}_{\gamma}$ & $(0.084722)$ & $(0.069792)$ & $(0.060328)$ & $(0.051640)$ \\
\hline & & & $\hat{F}_{\delta}$ & 0.06799214 & 0.24448450 & 0.49317147 & 0.75309426 \\
\hline & & & $\hat{\sigma}_{\delta}$ & $(0.063831)$ & $(0.075284)$ & $(0.072030)$ & $(0.060368)$ \\
\hline & & & $\hat{F}_{c o r}$ & 0.09701878 & 0.25150564 & 0.49198294 & 0.74489399 \\
\hline & & & $\hat{\sigma}_{c o r}$ & $(0.101966)$ & $(0.114552)$ & $(0.100547)$ & $(0.086067)$ \\
\hline \multirow[t]{6}{*}{500} & 0.7 & 0.9 & $\hat{F}_{\gamma}$ & 0.13880650 & 0.24469530 & 0.39300460 & 0.54510120 \\
\hline & & & $\hat{\sigma}_{\gamma}$ & $(0.067180)$ & $(0.065501)$ & $(0.060387)$ & $(0.055969)$ \\
\hline & & & $\hat{F}_{\delta}$ & 0.06799214 & 0.24448450 & 0.49317147 & 0.75309426 \\
\hline & & & $\hat{\sigma}_{\delta}$ & $(0.063831)$ & $(0.075284)$ & $(0.072030)$ & $(0.060368)$ \\
\hline & & & $\hat{F}_{c o r}$ & 0.08417352 & 0.24144534 & 0.48834108 & 0.74183535 \\
\hline & & & $\hat{\sigma}_{c o r}$ & $(0.085414)$ & $(0.108473)$ & $(0.100645)$ & $(0.093283)$ \\
\hline \multirow[t]{6}{*}{500} & 0.9 & 0.9 & $\hat{F}_{\gamma}$ & 0.15794720 & 0.29598880 & 0.49431500 & 0.69706770 \\
\hline & & & $\hat{\sigma}_{\gamma}$ & $(0.075897)$ & $(0.074524)$ & $(0.067319)$ & $(0.058669)$ \\
\hline & & & $\hat{F}_{\delta}$ & 0.06799214 & 0.24448450 & 0.49317147 & 0.75309426 \\
\hline & & & $\hat{\sigma}_{\delta}$ & $(0.063831)$ & $(0.075284)$ & $(0.072030)$ & $(0.060368)$ \\
\hline & & & $\hat{F}_{c o r}$ & 0.08431778 & 0.24504222 & 0.49289372 & 0.74633463 \\
\hline & & & $\hat{\sigma}_{c o r}$ & $(0.077822)$ & $(0.092999)$ & $(0.084149)$ & $(0.073336)$ \\
\hline
\end{tabular}


Tabela 4.9: Estimação da esperança e erro-padrão de $F_{\gamma}, F_{\delta}$ e $F_{c o r}$ no quantil da T, SEM suavização, com $70 \%$ de censura à esquerda e 30\% à direita

\begin{tabular}{|c|c|c|c|c|c|c|c|}
\hline \multirow[b]{2}{*}{$\mathrm{n}$} & \multirow[b]{2}{*}{$\mathrm{s}$} & \multirow[b]{2}{*}{ e } & & \multicolumn{2}{|l|}{ Quantil } & \multirow[b]{2}{*}{0.5} & \multirow[b]{2}{*}{0.75} \\
\hline & & & & 0.10 & 0.25 & & \\
\hline \multirow[t]{6}{*}{1000} & 0.7 & 0.7 & $\hat{F}_{\gamma}$ & 0.32595970 & 0.39456670 & 0.49991210 & 0.59706360 \\
\hline & & & $\hat{\sigma}_{\gamma}$ & $(0.055451)$ & $(0.048062)$ & $(0.046398)$ & $(0.037525)$ \\
\hline & & & $\hat{F}_{\delta}$ & 0.07977697 & 0.24031245 & 0.49759355 & 0.75168251 \\
\hline & & & $\hat{\sigma}_{\delta}$ & $(0.051000)$ & $(0.058855)$ & $(0.058714)$ & $(0.048788)$ \\
\hline & & & $\hat{F}_{c o r}$ & 0.09480381 & 0.23756458 & 0.49978026 & 0.74265898 \\
\hline & & & $\hat{\sigma}_{c o r}$ & $(0.097114)$ & $(0.117462)$ & $(0.115995)$ & $(0.093812)$ \\
\hline \multirow[t]{6}{*}{1000} & 0.9 & 0.7 & $\hat{F}_{\gamma}$ & 0.34860340 & 0.44714420 & 0.60230730 & 0.74864490 \\
\hline & & & $\hat{\sigma}_{\gamma}$ & $(0.062005)$ & $(0.055621)$ & $(0.052146)$ & $(0.039498)$ \\
\hline & & & $\hat{F}_{\delta}$ & 0.07977697 & 0.24031245 & 0.49759355 & 0.75168251 \\
\hline & & & $\hat{\sigma}_{\delta}$ & $(0.051000)$ & $(0.058855)$ & $(0.058714)$ & $(0.048788)$ \\
\hline & & & $\hat{F}_{c o r}$ & 0.09469966 & 0.24537609 & 0.50384552 & 0.74774152 \\
\hline & & & $\hat{\sigma}_{c o r}$ & $(0.082348)$ & $(0.092309)$ & $(0.086911)$ & $(0.065830)$ \\
\hline \multirow[t]{6}{*}{1000} & 0.7 & 0.9 & $\hat{F}_{\gamma}$ & 0.14681190 & 0.24236670 & 0.39966090 & 0.54742460 \\
\hline & & & $\hat{\sigma}_{\gamma}$ & $(0.048501)$ & $(0.050661)$ & $(0.049816)$ & $(0.044427)$ \\
\hline & & & $\hat{F}_{\delta}$ & 0.07977697 & 0.24031245 & 0.49759355 & 0.75168251 \\
\hline & & & $\hat{\sigma}_{\delta}$ & $(0.051000)$ & $(0.058855)$ & $(0.058714)$ & $(0.048788)$ \\
\hline & & & $\hat{F}_{c o r}$ & 0.08461450 & 0.23734530 & 0.49943480 & 0.74570760 \\
\hline & & & $\hat{\sigma}_{c o r}$ & $(0.071153)$ & $(0.084232)$ & $(0.083026)$ & $(0.074045)$ \\
\hline \multirow[t]{6}{*}{1000} & 0.9 & 0.9 & $\hat{F}_{\gamma}$ & 0.16639060 & 0.29414330 & 0.50127580 & 0.70005440 \\
\hline & & & $\hat{\sigma}_{\gamma}$ & $(0.055615)$ & $(0.057582)$ & $(0.055489)$ & $(0.045905)$ \\
\hline & & & $\hat{F}_{\delta}$ & 0.07977697 & 0.24031245 & 0.49759355 & 0.75168251 \\
\hline & & & $\hat{\sigma}_{\delta}$ & $(0.051000)$ & $(0.058855)$ & $(0.058714)$ & $(0.048788)$ \\
\hline & & & $\hat{F}_{c o r}$ & 0.08613736 & 0.24268732 & 0.50159479 & 0.75006798 \\
\hline & & & $\hat{\sigma}_{c o r}$ & $(0.064415)$ & $(0.071949)$ & $(0.069361)$ & $(0.057381)$ \\
\hline
\end{tabular}


Tabela 4.10: Estimação da esperança e erro-padrão de $F_{\gamma}, F_{\delta}$ e $F_{c o r}$ no quantil da T, COM suavização, com $70 \%$ de censura à esquerda e 30\% à direita

\begin{tabular}{|c|c|c|c|c|c|c|c|}
\hline \multirow[b]{2}{*}{$\mathrm{n}$} & \multirow[b]{2}{*}{$\mathrm{s}$} & \multirow[b]{2}{*}{ e } & & \multicolumn{2}{|l|}{ Quantil } & \multirow[b]{2}{*}{0.5} & \multirow[b]{2}{*}{0.75} \\
\hline & & & & 0.10 & 0.25 & & \\
\hline \multirow[t]{6}{*}{200} & 0.7 & 0.7 & $\hat{F}_{\gamma}$ & 0.25674150 & 0.37324790 & 0.48719410 & 0.58621470 \\
\hline & & & $\hat{\sigma}_{\gamma}$ & $(0.072854)$ & $(0.064266)$ & $(0.054056)$ & $(0.047274)$ \\
\hline & & & $\hat{F}_{\delta}$ & 0.09341417 & 0.23593636 & 0.48484144 & 0.73963693 \\
\hline & & & $\hat{\sigma}_{\delta}$ & $(0.038943)$ & $(0.054668)$ & $(0.056987)$ & $(0.047863)$ \\
\hline & & & $\hat{F}_{\text {cor }}$ & 0.12190440 & 0.24842300 & 0.47356710 & 0.71156640 \\
\hline & & & $\hat{\sigma}_{c o r}$ & $(0.100700)$ & $(0.127239)$ & $(0.131690)$ & $(0.114136)$ \\
\hline \multirow[t]{6}{*}{200} & 0.9 & 0.7 & $\hat{F}_{\gamma}$ & 0.28605680 & 0.43081690 & 0.59181310 & 0.74088210 \\
\hline & & & $\hat{\sigma}_{\gamma}$ & $(0.076931)$ & $(0.067318)$ & $(0.054079)$ & $(0.044398)$ \\
\hline & & & $\hat{F}_{\delta}$ & 0.09341417 & 0.23593636 & 0.48484144 & 0.73963693 \\
\hline & & & $\hat{\sigma}_{\delta}$ & $(0.038943)$ & $(0.054668)$ & $(0.056987)$ & $(0.047863)$ \\
\hline & & & $\hat{F}_{c o r}$ & 0.12251160 & 0.25520000 & 0.48861930 & 0.73424430 \\
\hline & & & $\hat{\sigma}_{c o r}$ & $(0.079263)$ & $(0.095867)$ & $(0.089449)$ & $(0.073658)$ \\
\hline \multirow[t]{6}{*}{200} & 0.7 & 0.9 & $\hat{F}_{\gamma}$ & 0.12333090 & 0.23045410 & 0.38438590 & 0.53287640 \\
\hline & & & $\hat{\sigma}_{\gamma}$ & $(0.052708)$ & $(0.057238)$ & $(0.054085)$ & $(0.049720)$ \\
\hline & & & $\hat{F}_{\delta}$ & 0.09341417 & 0.23593636 & 0.48484144 & 0.73963693 \\
\hline & & & $\hat{\sigma}_{\delta}$ & $(0.038943)$ & $(0.054668)$ & $(0.056987)$ & $(0.047863)$ \\
\hline & & & $\hat{F}_{c o r}$ & 0.10725300 & 0.23893090 & 0.47543190 & 0.72013810 \\
\hline & & & $\hat{\sigma}_{c o r}$ & $(0.066565)$ & $(0.086387)$ & $(0.089815)$ & $(0.081866)$ \\
\hline \multirow[t]{6}{*}{200} & 0.9 & 0.9 & $\hat{F}_{\gamma}$ & 0.14794850 & 0.28451740 & 0.48786800 & 0.68745320 \\
\hline & & & $\hat{\sigma}_{\gamma}$ & $(0.056928)$ & $(0.061329)$ & $(0.055554)$ & $(0.047731)$ \\
\hline & & & $\hat{F}_{\delta}$ & 0.09341417 & 0.23593636 & 0.48484144 & 0.73963693 \\
\hline & & & $\hat{\sigma}_{\delta}$ & $(0.038943)$ & $(0.054668)$ & $(0.056987)$ & $(0.047863)$ \\
\hline & & & $\hat{F}_{c o r}$ & 0.10909520 & 0.24508600 & 0.48564710 & 0.73409850 \\
\hline & & & $\hat{\sigma}_{c o r}$ & $(0.056514)$ & $(0.071466)$ & $(0.069283)$ & $(0.059558)$ \\
\hline
\end{tabular}


Tabela 4.11: Estimação da esperança e erro-padrão de $F_{\gamma}, F_{\delta}$ e $F_{c o r}$ no quantil da T, COM suavização, com $70 \%$ de censura à esquerda e 30\% à direita

\begin{tabular}{|c|c|c|c|c|c|c|c|}
\hline \multirow[b]{2}{*}{$\mathrm{n}$} & \multirow[b]{2}{*}{$\mathrm{s}$} & \multirow[b]{2}{*}{ e } & & \multicolumn{2}{|l|}{ Quantil } & \multirow[b]{2}{*}{0.5} & \multirow[b]{2}{*}{0.75} \\
\hline & & & & 0.10 & 0.25 & & \\
\hline \multirow[t]{6}{*}{500} & 0.7 & 0.7 & $\hat{F}_{\gamma}$ & 0.29544520 & 0.39294130 & 0.49307810 & 0.59006200 \\
\hline & & & $\hat{\sigma}_{\gamma}$ & $(0.053179)$ & $(0.043975)$ & $(0.035955)$ & $(0.034193)$ \\
\hline & & & $\hat{F}_{\delta}$ & 0.09640220 & 0.24656720 & 0.49240210 & 0.74095970 \\
\hline & & & $\hat{\sigma}_{\delta}$ & $(0.029465)$ & $(0.038303)$ & $(0.038597)$ & $(0.032392)$ \\
\hline & & & $\hat{F}_{c o r}$ & 0.11816850 & 0.25313970 & 0.48328810 & 0.72448260 \\
\hline & & & $\hat{\sigma}_{c o r}$ & $(0.082847)$ & $(0.099217)$ & $(0.089532)$ & $(0.084777)$ \\
\hline \multirow[t]{6}{*}{500} & 0.9 & 0.7 & $\hat{F}_{\gamma}$ & 0.32216800 & 0.44785420 & 0.59455200 & 0.74091650 \\
\hline & & & $\hat{\sigma}_{\gamma}$ & $(0.055450)$ & $(0.045689)$ & $(0.037267)$ & $(0.032588)$ \\
\hline & & & $\hat{F}_{\delta}$ & 0.09640220 & 0.24656720 & 0.49240210 & 0.74095970 \\
\hline & & & $\hat{\sigma}_{\delta}$ & $(0.029465)$ & $(0.038303)$ & $(0.038597)$ & $(0.032392)$ \\
\hline & & & $\hat{F}_{\text {cor }}$ & 0.11713160 & 0.25698030 & 0.49108110 & 0.73480640 \\
\hline & & & $\hat{\sigma}_{c o r}$ & $(0.063869)$ & $(0.072014)$ & $(0.062080)$ & $(0.054288)$ \\
\hline \multirow[t]{6}{*}{500} & 0.7 & 0.9 & $\hat{F}_{\gamma}$ & 0.13810270 & 0.24409610 & 0.39144320 & 0.53793100 \\
\hline & & & $\hat{\sigma}_{\gamma}$ & $(0.039133)$ & $(0.039351)$ & $(0.036814)$ & $(0.036074)$ \\
\hline & & & $\hat{F}_{\delta}$ & 0.09640220 & 0.24656720 & 0.49240210 & 0.74095970 \\
\hline & & & $\hat{\sigma}_{\delta}$ & $(0.029465)$ & $(0.038303)$ & $(0.038597)$ & $(0.032392)$ \\
\hline & & & $\hat{F}_{c o r}$ & 0.10495700 & 0.24649530 & 0.48582710 & 0.72973440 \\
\hline & & & $\hat{\sigma}_{c o r}$ & $(0.051135)$ & $(0.063748)$ & $(0.061350)$ & $(0.060011)$ \\
\hline \multirow[t]{6}{*}{500} & 0.9 & 0.9 & $\hat{F}_{\gamma}$ & 0.16160120 & 0.29701800 & 0.49266010 & 0.68851300 \\
\hline & & & $\hat{\sigma}_{\gamma}$ & $(0.042057)$ & $(0.041961)$ & $(0.038626)$ & $(0.034848)$ \\
\hline & & & $\hat{F}_{\delta}$ & 0.09640220 & 0.24656720 & 0.49240210 & 0.74095970 \\
\hline & & & $\hat{\sigma}_{\delta}$ & $(0.029465)$ & $(0.038303)$ & $(0.038597)$ & $(0.032392)$ \\
\hline & & & $\hat{F}_{c o r}$ & 0.10637710 & 0.25034920 & 0.49086740 & 0.73562410 \\
\hline & & & $\hat{\sigma}_{c o r}$ & $(0.043645)$ & $(0.051605)$ & $(0.048285)$ & $(0.043560)$ \\
\hline
\end{tabular}


Tabela 4.12: Estimação da esperança e erro-padrão de $F_{\gamma}, F_{\delta}$ e $F_{c o r}$ no quantil da T, COM suavização, com $70 \%$ de censura à esquerda e $30 \%$ à direita

\begin{tabular}{|c|c|c|c|c|c|c|c|}
\hline \multirow[b]{2}{*}{$\mathrm{n}$} & \multirow[b]{2}{*}{$\mathrm{s}$} & \multirow[b]{2}{*}{ e } & & \multicolumn{2}{|l|}{ Quantil } & \multirow[b]{2}{*}{0.5} & \multirow[b]{2}{*}{0.75} \\
\hline & & & & 0.10 & 0.25 & & \\
\hline \multirow[t]{6}{*}{1000} & 0.7 & 0.7 & $\hat{F}_{\gamma}$ & 0.31272600 & 0.39462790 & 0.49652070 & 0.59385010 \\
\hline & & & $\hat{\sigma}_{\gamma}$ & $(0.039771)$ & $(0.032155)$ & $(0.029543)$ & $(0.025844)$ \\
\hline & & & $\hat{F}_{\delta}$ & 0.09719025 & 0.24646975 & 0.49355582 & 0.74247156 \\
\hline & & & $\hat{\sigma}_{\delta}$ & $(0.024038)$ & $(0.028599)$ & $(0.029874)$ & $(0.026118)$ \\
\hline & & & $\hat{F}_{\text {cor }}$ & 0.10786700 & 0.24450380 & 0.49139540 & 0.73442630 \\
\hline & & & $\hat{\sigma}_{c o r}$ & $(0.066892)$ & $(0.076148)$ & $(0.073835)$ & $(0.064458)$ \\
\hline \multirow[t]{6}{*}{1000} & 0.9 & 0.7 & $\hat{F}_{\gamma}$ & 0.33829520 & 0.44794060 & 0.59740700 & 0.74406400 \\
\hline & & & $\hat{\sigma}_{\gamma}$ & $(0.041661)$ & $(0.033470)$ & $(0.029876)$ & $(0.024770)$ \\
\hline & & & $\hat{F}_{\delta}$ & 0.09719025 & 0.24646975 & 0.49355582 & 0.74247156 \\
\hline & & & $\hat{\sigma}_{\delta}$ & $(0.024038)$ & $(0.028599)$ & $(0.029874)$ & $(0.026118)$ \\
\hline & & & $\hat{F}_{c o r}$ & 0.10931270 & 0.25002030 & 0.49569630 & 0.74010120 \\
\hline & & & $\hat{\sigma}_{c o r}$ & $(0.052137)$ & $(0.054406)$ & $(0.049790)$ & $(0.041284)$ \\
\hline \multirow[t]{6}{*}{1000} & 0.7 & 0.9 & $\hat{F}_{\gamma}$ & 0.14658540 & 0.24496600 & 0.39462040 & 0.54235940 \\
\hline & & & $\hat{\sigma}_{\gamma}$ & $(0.030209)$ & $(0.028454)$ & $(0.029169)$ & $(0.028060)$ \\
\hline & & & $\hat{F}_{\delta}$ & 0.09719025 & 0.24646975 & 0.49355582 & 0.74247156 \\
\hline & & & $\hat{\sigma}_{\delta}$ & $(0.024038)$ & $(0.028599)$ & $(0.029874)$ & $(0.026118)$ \\
\hline & & & $\hat{F}_{c o r}$ & 0.10140160 & 0.24344000 & 0.49104040 & 0.73723980 \\
\hline & & & $\hat{\sigma}_{c o r}$ & $(0.042563)$ & $(0.046964)$ & $(0.048615)$ & $(0.046756)$ \\
\hline \multirow[t]{6}{*}{1000} & 0.9 & 0.9 & $\hat{F}_{\gamma}$ & 0.16956290 & 0.29743850 & 0.49517550 & 0.69253930 \\
\hline & & & $\hat{\sigma}_{\gamma}$ & $(0.031827)$ & $(0.030780)$ & $(0.030244)$ & $(0.027204)$ \\
\hline & & & $\hat{F}_{\delta}$ & 0.09719025 & 0.24646975 & 0.49355582 & 0.74247156 \\
\hline & & & $\hat{\sigma}_{\delta}$ & $(0.024038)$ & $(0.028599)$ & $(0.029874)$ & $(0.026118)$ \\
\hline & & & $\hat{F}_{c o r}$ & 0.10317060 & 0.24783010 & 0.49397160 & 0.74067290 \\
\hline & & & $\hat{\sigma}_{c o r}$ & $(0.035266)$ & $(0.038262)$ & $(0.037805)$ & $(0.034005)$ \\
\hline
\end{tabular}


Tabela 4.13: Estimação da esperança e erro-padrão de $F_{\gamma}, F_{\delta}$ e $F_{c o r}$ no quantil da T, SEM suavização, com 30\% de censura à esquerda e 70\% à direita

\begin{tabular}{|c|c|c|c|c|c|c|c|}
\hline \multirow[b]{2}{*}{$\mathrm{n}$} & \multirow[b]{2}{*}{$\mathrm{S}$} & \multirow[b]{2}{*}{ e } & & \multicolumn{2}{|l|}{ Quantil } & \multirow[b]{2}{*}{0.5} & \multirow[b]{2}{*}{0.75} \\
\hline & & & & 0.10 & 0.25 & & \\
\hline \multirow[t]{6}{*}{200} & 0.7 & 0.7 & $\hat{F}_{\gamma}$ & 0.33082640 & 0.39924520 & 0.50871020 & 0.64770150 \\
\hline & & & $\hat{\sigma}_{\gamma}$ & $(0.061579)$ & $(0.061185)$ & $(0.068599)$ & $(0.113547)$ \\
\hline & & & $\hat{F}_{\delta}$ & 0.08198045 & 0.24551973 & 0.50463637 & 0.79168771 \\
\hline & & & $\hat{\sigma}_{\delta}$ & $(0.053027)$ & $(0.071245)$ & $(0.095491)$ & $(0.127732)$ \\
\hline & & & $\hat{F}_{\text {cor }}$ & 0.10799070 & 0.25097090 & 0.52142350 & 0.80563870 \\
\hline & & & $\hat{\sigma}_{c o r}$ & $(0.111850)$ & $(0.147250)$ & $(0.170432)$ & $(0.186337)$ \\
\hline \multirow[t]{6}{*}{200} & 0.9 & 0.7 & $\hat{F}_{\gamma}$ & 0.35319150 & 0.45221740 & 0.60856030 & 0.79509600 \\
\hline & & & $\hat{\sigma}_{\gamma}$ & $(0.067285)$ & $(0.068360)$ & $(0.076990)$ & $(0.112109)$ \\
\hline & & & $\hat{F}_{\delta}$ & 0.08198045 & 0.24551973 & 0.50463637 & 0.79168771 \\
\hline & & & $\hat{\sigma}_{\delta}$ & $(0.053027)$ & $(0.071245)$ & $(0.095491)$ & $(0.127732)$ \\
\hline & & & $\hat{F}_{c o r}$ & 0.10268960 & 0.25416400 & 0.51426720 & 0.80323450 \\
\hline & & & $\hat{\sigma}_{c o r}$ & $(0.091058)$ & $(0.112748)$ & $(0.128318)$ & $(0.152945)$ \\
\hline \multirow[t]{6}{*}{200} & 0.7 & 0.9 & $\hat{F}_{\gamma}$ & 0.15032570 & 0.24675290 & 0.40398790 & 0.58959700 \\
\hline & & & $\hat{\sigma}_{\gamma}$ & $(0.052900)$ & $(0.061832)$ & $(0.076634)$ & $(0.125185)$ \\
\hline & & & $\hat{F}_{\delta}$ & 0.08198045 & 0.24551973 & 0.50463637 & 0.79168771 \\
\hline & & & $\hat{\sigma}_{\delta}$ & $(0.053027)$ & $(0.071245)$ & $(0.095491)$ & $(0.127732)$ \\
\hline & & & $\hat{F}_{c o r}$ & 0.09102079 & 0.24468727 & 0.50664654 & 0.79001470 \\
\hline & & & $\hat{\sigma}_{c o r}$ & $(0.077045)$ & $(0.102793)$ & $(0.127724)$ & $(0.162456)$ \\
\hline \multirow[t]{6}{*}{200} & 0.9 & 0.9 & $\hat{F}_{\gamma}$ & 0.16985250 & 0.29892670 & 0.50518330 & 0.74116000 \\
\hline & & & $\hat{\sigma}_{\gamma}$ & $(0.058402)$ & $(0.071221)$ & $(0.086173)$ & $(0.127812)$ \\
\hline & & & $\hat{F}_{\delta}$ & 0.08198045 & 0.24551973 & 0.50463637 & 0.79168771 \\
\hline & & & $\hat{\sigma}_{\delta}$ & $(0.053027)$ & $(0.071245)$ & $(0.095491)$ & $(0.127732)$ \\
\hline & & & $\hat{F}_{c o r}$ & 0.09112069 & 0.24865838 & 0.50647909 & 0.79038259 \\
\hline & & & $\hat{\sigma}_{c o r}$ & $(0.066373)$ & $(0.089027)$ & $(0.107717)$ & $(0.140314)$ \\
\hline
\end{tabular}


Tabela 4.14: Estimação da esperança e erro-padrão de $F_{\gamma}, F_{\delta}$ e $F_{c o r}$ no quantil da T, SEM suavização, com 30\% de censura à esquerda e 70\% à direita

\begin{tabular}{|c|c|c|c|c|c|c|c|}
\hline \multirow[b]{2}{*}{$\mathrm{n}$} & \multirow[b]{2}{*}{$\mathrm{s}$} & \multirow[b]{2}{*}{ e } & & \multicolumn{2}{|l|}{ Quantil } & \multirow[b]{2}{*}{0.5} & \multirow[b]{2}{*}{0.75} \\
\hline & & & & 0.10 & 0.25 & & \\
\hline \multirow[t]{6}{*}{500} & 0.7 & 0.7 & $\hat{F}_{\gamma}$ & 0.33521850 & 0.40195700 & 0.50298830 & 0.62709800 \\
\hline & & & $\hat{\sigma}_{\gamma}$ & $(0.039930)$ & $(0.042457)$ & $(0.050040)$ & $(0.075347)$ \\
\hline & & & $\hat{F}_{\delta}$ & 0.09078429 & 0.24739962 & 0.50659051 & 0.77461434 \\
\hline & & & $\hat{\sigma}_{\delta}$ & $(0.035100)$ & $(0.051234)$ & $(0.069950)$ & $(0.085413)$ \\
\hline & & & $\hat{F}_{c o r}$ & 0.09802439 & 0.25489986 & 0.50747082 & 0.79864004 \\
\hline & & & $\hat{\sigma}_{c o r}$ & $(0.084378)$ & $(0.106124)$ & $(0.125100)$ & $(0.154911)$ \\
\hline \multirow[t]{6}{*}{500} & 0.9 & 0.7 & $\hat{F}_{\gamma}$ & 0.35646400 & 0.45300120 & 0.60510240 & 0.77176000 \\
\hline & & & $\hat{\sigma}_{\gamma}$ & $(0.043285)$ & $(0.049227)$ & $(0.056506)$ & $(0.072746)$ \\
\hline & & & $\hat{F}_{\delta}$ & 0.09078429 & 0.24739962 & 0.50659051 & 0.77461434 \\
\hline & & & $\hat{\sigma}_{\delta}$ & $(0.035100)$ & $(0.051234)$ & $(0.069950)$ & $(0.085413)$ \\
\hline & & & $\hat{F}_{c o r}$ & 0.09738567 & 0.25500202 & 0.50850408 & 0.78346899 \\
\hline & & & $\hat{\sigma}_{c o r}$ & $(0.066178)$ & $(0.082045)$ & $(0.094176)$ & $(0.114946)$ \\
\hline \multirow[t]{6}{*}{500} & 0.7 & 0.9 & $\hat{F}_{\gamma}$ & 0.15463650 & 0.24839800 & 0.40222560 & 0.57189020 \\
\hline & & & $\hat{\sigma}_{\gamma}$ & $(0.036591)$ & $(0.044419)$ & $(0.058293)$ & $(0.081864)$ \\
\hline & & & $\hat{F}_{\delta}$ & 0.09078429 & 0.24739962 & 0.50659051 & 0.77461434 \\
\hline & & & $\hat{\sigma}_{\delta}$ & $(0.035100)$ & $(0.051234)$ & $(0.069950)$ & $(0.085413)$ \\
\hline & & & $\hat{F}_{c o r}$ & 0.09237472 & 0.24733004 & 0.50370929 & 0.78176703 \\
\hline & & & $\hat{\sigma}_{c o r}$ & $(0.058557)$ & $(0.074032)$ & $(0.097155)$ & $(0.126059)$ \\
\hline \multirow[t]{6}{*}{500} & 0.9 & 0.9 & $\hat{F}_{\gamma}$ & 0.17412660 & 0.29965310 & 0.50572160 & 0.72029000 \\
\hline & & & $\hat{\sigma}_{\gamma}$ & $(0.040012)$ & $(0.050996)$ & $(0.064121)$ & $(0.082252)$ \\
\hline & & & $\hat{F}_{\delta}$ & 0.09078429 & 0.24739962 & 0.50659051 & 0.77461434 \\
\hline & & & $\hat{\sigma}_{\delta}$ & $(0.035100)$ & $(0.051234)$ & $(0.069950)$ & $(0.085413)$ \\
\hline & & & $\hat{F}_{c o r}$ & 0.09302228 & 0.24956632 & 0.50715199 & 0.77431669 \\
\hline & & & $\hat{\sigma}_{c o r}$ & $(0.049241)$ & $(0.063745)$ & $(0.080151)$ & $(0.100049)$ \\
\hline
\end{tabular}


Tabela 4.15: Estimação da esperança e erro-padrão de $F_{\gamma}, F_{\delta}$ e $F_{c o r}$ no quantil da T, SEM suavização, com 30\% de censura à esquerda e 70\% à direita

\begin{tabular}{|c|c|c|c|c|c|c|c|}
\hline \multirow[b]{2}{*}{$\mathrm{n}$} & \multirow[b]{2}{*}{$\mathrm{S}$} & \multirow[b]{2}{*}{ e } & & \multicolumn{2}{|l|}{ Quantil } & \multirow[b]{2}{*}{0.5} & \multirow[b]{2}{*}{0.75} \\
\hline & & & & 0.10 & 0.25 & & \\
\hline \multirow[t]{6}{*}{1000} & 0.7 & 0.7 & $\hat{F}_{\gamma}$ & 0.33779410 & 0.40001150 & 0.50198360 & 0.61762930 \\
\hline & & & $\hat{\sigma}_{\gamma}$ & $(0.031389)$ & $(0.032428)$ & $(0.039573)$ & $(0.058252)$ \\
\hline & & & $\hat{F}_{\delta}$ & 0.09341661 & 0.24881832 & 0.50001535 & 0.76658855 \\
\hline & & & $\hat{\sigma}_{\delta}$ & $(0.029007)$ & $(0.041884)$ & $(0.053125)$ & $(0.067362)$ \\
\hline & & & $\hat{F}_{\text {cor }}$ & 0.09922030 & 0.25002880 & 0.50495890 & 0.78750590 \\
\hline & & & $\hat{\sigma}_{c o r}$ & $(0.070059)$ & $(0.081071)$ & $(0.098934)$ & $(0.132597)$ \\
\hline \multirow[t]{6}{*}{1000} & 0.9 & 0.7 & $\hat{F}_{\gamma}$ & 0.35916220 & 0.45132220 & 0.60111850 & 0.76579750 \\
\hline & & & $\hat{\sigma}_{\gamma}$ & $(0.035137)$ & $(0.038024)$ & $(0.044400)$ & $(0.057109)$ \\
\hline & & & $\hat{F}_{\delta}$ & 0.09341661 & 0.24881832 & 0.50001535 & 0.76658855 \\
\hline & & & $\hat{\sigma}_{\delta}$ & $(0.029007)$ & $(0.041884)$ & $(0.053125)$ & $(0.067362)$ \\
\hline & & & $\hat{F}_{c o r}$ & 0.09982390 & 0.25220360 & 0.50186420 & 0.77592160 \\
\hline & & & $\hat{\sigma}_{c o r}$ & $(0.055988)$ & $(0.063374)$ & $(0.074001)$ & $(0.094105)$ \\
\hline \multirow[t]{6}{*}{1000} & 0.7 & 0.9 & $\hat{F}_{\gamma}$ & 0.15637650 & 0.24828690 & 0.40114510 & 0.56436920 \\
\hline & & & $\hat{\sigma}_{\gamma}$ & $(0.029498)$ & $(0.033859)$ & $(0.045399)$ & $(0.064925)$ \\
\hline & & & $\hat{F}_{\delta}$ & 0.09341661 & 0.24881832 & 0.50001535 & 0.76658855 \\
\hline & & & $\hat{\sigma}_{\delta}$ & $(0.029007)$ & $(0.041884)$ & $(0.053125)$ & $(0.067362)$ \\
\hline & & & $\hat{F}_{c o r}$ & 0.09438857 & 0.24714487 & 0.50190858 & 0.77278868 \\
\hline & & & $\hat{\sigma}_{c o r}$ & $(0.048204)$ & $(0.056432)$ & $(0.075665)$ & $(0.105212)$ \\
\hline \multirow[t]{6}{*}{1000} & 0.9 & 0.9 & $\hat{F}_{\gamma}$ & 0.17706080 & 0.29892530 & 0.50054090 & 0.71426670 \\
\hline & & & $\hat{\sigma}_{\gamma}$ & $(0.033090)$ & $(0.039751)$ & $(0.049520)$ & $(0.065466)$ \\
\hline & & & $\hat{F}_{\delta}$ & 0.09341661 & 0.24881832 & 0.50001535 & 0.76658855 \\
\hline & & & $\hat{\sigma}_{\delta}$ & $(0.029007)$ & $(0.041884)$ & $(0.053125)$ & $(0.067362)$ \\
\hline & & & $\hat{F}_{c o r}$ & 0.09639274 & 0.24865656 & 0.50067613 & 0.76775451 \\
\hline & & & $\hat{\sigma}_{c o r}$ & $(0.041190)$ & $(0.049689)$ & $(0.061901)$ & $(0.081599)$ \\
\hline
\end{tabular}


Tabela 4.16: Estimação da esperança e erro-padrão de $F_{\gamma}, F_{\delta}$ e $F_{c o r}$ no quantil da T, COM suavização, com $30 \%$ de censura à esquerda e $70 \%$ à direita

\begin{tabular}{|c|c|c|c|c|c|c|c|}
\hline \multirow[b]{2}{*}{$\mathrm{n}$} & \multirow[b]{2}{*}{$\mathrm{s}$} & \multirow[b]{2}{*}{ e } & & \multicolumn{2}{|l|}{ Quantil } & \multirow[b]{2}{*}{0.5} & \multirow[b]{2}{*}{0.75} \\
\hline & & & & 0.10 & 0.25 & & \\
\hline \multirow[t]{6}{*}{200} & 0.7 & 0.7 & $\hat{F}_{\gamma}$ & 0.31975760 & 0.40157350 & 0.50979370 & 0.65437560 \\
\hline & & & $\hat{\sigma}_{\gamma}$ & $(0.046716)$ & $(0.044215)$ & $(0.051994)$ & $(0.090448)$ \\
\hline & & & $\hat{F}_{\delta}$ & 0.09651403 & 0.24893294 & 0.50366648 & 0.78764483 \\
\hline & & & $\hat{\sigma}_{\delta}$ & $(0.028361)$ & $(0.039673)$ & $(0.056698)$ & $(0.086646)$ \\
\hline & & & $\hat{F}_{c o r}$ & 0.11720900 & 0.26130280 & 0.52160720 & 0.79549600 \\
\hline & & & $\hat{\sigma}_{c o r}$ & $(0.083680)$ & $(0.105301)$ & $(0.127945)$ & $(0.147582)$ \\
\hline \multirow[t]{6}{*}{200} & 0.9 & 0.7 & $\hat{F}_{\gamma}$ & 0.34483760 & 0.45449250 & 0.60905150 & 0.79429860 \\
\hline & & & $\hat{\sigma}_{\gamma}$ & $(0.048763)$ & $(0.045577)$ & $(0.052830)$ & $(0.081723)$ \\
\hline & & & $\hat{F}_{\delta}$ & 0.09651403 & 0.24893294 & 0.50366648 & 0.78764483 \\
\hline & & & $\hat{\sigma}_{\delta}$ & $(0.028361)$ & $(0.039673)$ & $(0.056698)$ & $(0.086646)$ \\
\hline & & & $\hat{F}_{\text {cor }}$ & 0.11448370 & 0.26032680 & 0.51462120 & 0.79333280 \\
\hline & & & $\hat{\sigma}_{c o r}$ & $(0.063681)$ & $(0.074399)$ & $(0.087834)$ & $(0.113639)$ \\
\hline \multirow[t]{6}{*}{200} & 0.7 & 0.9 & $\hat{F}_{\gamma}$ & 0.15024810 & 0.24983680 & 0.40396340 & 0.59477500 \\
\hline & & & $\hat{\sigma}_{\gamma}$ & $(0.035349)$ & $(0.038914)$ & $(0.053151)$ & $(0.097508)$ \\
\hline & & & $\hat{F}_{\delta}$ & 0.09651403 & 0.24893294 & 0.50366648 & 0.78764483 \\
\hline & & & $\hat{\sigma}_{\delta}$ & $(0.028361)$ & $(0.039673)$ & $(0.056698)$ & $(0.086646)$ \\
\hline & & & $\hat{F}_{c o r}$ & 0.10534640 & 0.25118470 & 0.50585050 & 0.77929390 \\
\hline & & & $\hat{\sigma}_{c o r}$ & $(0.050504)$ & $(0.064494)$ & $(0.088205)$ & $(0.123383)$ \\
\hline \multirow[t]{6}{*}{200} & 0.9 & 0.9 & $\hat{F}_{\gamma}$ & 0.17299730 & 0.30230050 & 0.50493100 & 0.73980380 \\
\hline & & & $\hat{\sigma}_{\gamma}$ & $(0.037723)$ & $(0.042210)$ & $(0.055156)$ & $(0.091906)$ \\
\hline & & & $\hat{F}_{\delta}$ & 0.09651403 & 0.24893294 & 0.50366648 & 0.78764483 \\
\hline & & & $\hat{\sigma}_{\delta}$ & $(0.028361)$ & $(0.039673)$ & $(0.056698)$ & $(0.086646)$ \\
\hline & & & $\hat{F}_{c o r}$ & 0.10600070 & 0.25365200 & 0.50596380 & 0.78138350 \\
\hline & & & $\hat{\sigma}_{c o r}$ & $(0.041966)$ & $(0.052627)$ & $(0.068876)$ & $(0.101072)$ \\
\hline
\end{tabular}


Tabela 4.17: Estimação da esperança e erro-padrão de $F_{\gamma}, F_{\delta}$ e $F_{c o r}$ no quantil da T, COM suavização, com $30 \%$ de censura à esquerda e $70 \%$ à direita

\begin{tabular}{|c|c|c|c|c|c|c|c|}
\hline \multirow[b]{2}{*}{$\mathrm{n}$} & \multirow[b]{2}{*}{$\mathrm{S}$} & \multirow[b]{2}{*}{$\mathrm{e}$} & & \multicolumn{2}{|l|}{ Quantil } & \multirow[b]{2}{*}{0.5} & \multirow[b]{2}{*}{0.75} \\
\hline & & & & 0.10 & 0.25 & & \\
\hline \multirow[t]{6}{*}{500} & 0.7 & 0.7 & $\hat{F}_{\gamma}$ & 0.33266020 & 0.40205580 & 0.50410620 & 0.62911490 \\
\hline & & & $\hat{\sigma}_{\gamma}$ & $(0.029116)$ & $(0.029142)$ & $(0.036313)$ & $(0.061864)$ \\
\hline & & & $\hat{F}_{\delta}$ & 0.09840856 & 0.24958429 & 0.50338117 & 0.77352166 \\
\hline & & & $\hat{\sigma}_{\delta}$ & $(0.019271)$ & $(0.027506)$ & $(0.040886)$ & $(0.059002)$ \\
\hline & & & $\hat{F}_{c o r}$ & 0.10768080 & 0.25627480 & 0.50999960 & 0.79022480 \\
\hline & & & $\hat{\sigma}_{\text {cor }}$ & $(0.059884)$ & $(0.072406)$ & $(0.090501)$ & $(0.124429)$ \\
\hline \multirow[t]{6}{*}{500} & 0.9 & 0.7 & $\hat{F}_{\gamma}$ & 0.35553490 & 0.45303910 & 0.60397830 & 0.77335290 \\
\hline & & & $\hat{\sigma}_{\gamma}$ & $(0.029656)$ & $(0.031064)$ & $(0.037283)$ & $(0.055384)$ \\
\hline & & & $\hat{F}_{\delta}$ & 0.09840856 & 0.24958429 & 0.50338117 & 0.77352166 \\
\hline & & & $\hat{\sigma}_{\delta}$ & $(0.019271)$ & $(0.027506)$ & $(0.040886)$ & $(0.059002)$ \\
\hline & & & $\hat{F}_{c o r}$ & 0.10670240 & 0.25539330 & 0.50661880 & 0.77972170 \\
\hline & & & $\hat{\sigma}_{\text {cor }}$ & $(0.043391)$ & $(0.051736)$ & $(0.062139)$ & $(0.085460)$ \\
\hline \multirow[t]{6}{*}{500} & 0.7 & 0.9 & $\hat{F}_{\gamma}$ & 0.15583190 & 0.24963680 & 0.40200580 & 0.57434330 \\
\hline & & & $\hat{\sigma}_{\gamma}$ & $(0.023779)$ & $(0.026265)$ & $(0.037953)$ & $(0.065641)$ \\
\hline & & & $\hat{F}_{\delta}$ & 0.09840856 & 0.24958429 & 0.50338117 & 0.77352166 \\
\hline & & & $\hat{\sigma}_{\delta}$ & $(0.019271)$ & $(0.027506)$ & $(0.040886)$ & $(0.059002)$ \\
\hline & & & $\hat{F}_{\text {cor }}$ & 0.10124410 & 0.24955020 & 0.50331390 & 0.77714190 \\
\hline & & & $\hat{\sigma}_{c o r}$ & $(0.036229)$ & $(0.043763)$ & $(0.063242)$ & $(0.097622)$ \\
\hline \multirow[t]{6}{*}{500} & 0.9 & 0.9 & $\hat{F}_{\gamma}$ & 0.17729410 & 0.30050220 & 0.50282240 & 0.72185570 \\
\hline & & & $\hat{\sigma}_{\gamma}$ & $(0.024697)$ & $(0.028641)$ & $(0.039697)$ & $(0.061295)$ \\
\hline & & & $\hat{F}_{\delta}$ & 0.09840856 & 0.24958429 & 0.50338117 & 0.77352166 \\
\hline & & & $\hat{\sigma}_{\delta}$ & $(0.019271)$ & $(0.027506)$ & $(0.040886)$ & $(0.059002)$ \\
\hline & & & $\hat{F}_{c o r}$ & 0.10178630 & 0.25069520 & 0.50352520 & 0.77234280 \\
\hline & & & $\hat{\sigma}_{c o r}$ & $(0.028986)$ & $(0.035797)$ & $(0.049622)$ & $(0.073092)$ \\
\hline
\end{tabular}


Tabela 4.18: Estimação da esperança e erro-padrão de $F_{\gamma}, F_{\delta}$ e $F_{c o r}$ no quantil da T, COM suavização, com $30 \%$ de censura à esquerda e $70 \%$ à direita

\begin{tabular}{|c|c|c|c|c|c|c|c|}
\hline \multirow[b]{2}{*}{$\mathrm{n}$} & \multirow[b]{2}{*}{$\mathrm{s}$} & \multirow[b]{2}{*}{ e } & & \multicolumn{2}{|l|}{ Quantil } & \multirow[b]{2}{*}{0.5} & \multirow[b]{2}{*}{0.75} \\
\hline & & & & 0.10 & 0.25 & & \\
\hline \multirow[t]{6}{*}{1000} & 0.7 & 0.7 & $\hat{F}_{\gamma}$ & 0.33780940 & 0.40161170 & 0.50193580 & 0.61655440 \\
\hline & & & $\hat{\sigma}_{\gamma}$ & $(0.023000)$ & $(0.022464)$ & $(0.027778)$ & $(0.046823)$ \\
\hline & & & $\hat{F}_{\delta}$ & 0.10027390 & 0.25064280 & 0.49995950 & 0.76271710 \\
\hline & & & $\hat{\sigma}_{\delta}$ & $(0.015127)$ & $(0.020992)$ & $(0.031381)$ & $(0.046096)$ \\
\hline & & & $\hat{F}_{\text {cor }}$ & 0.10682400 & 0.25427530 & 0.50481920 & 0.77957200 \\
\hline & & & $\hat{\sigma}_{c o r}$ & $(0.050143)$ & $(0.056054)$ & $(0.069431)$ & $(0.103678)$ \\
\hline \multirow[t]{6}{*}{1000} & 0.9 & 0.7 & $\hat{F}_{\gamma}$ & 0.36025920 & 0.45248230 & 0.60175080 & 0.76338870 \\
\hline & & & $\hat{\sigma}_{\gamma}$ & $(0.023671)$ & $(0.024076)$ & $(0.028867)$ & $(0.042992)$ \\
\hline & & & $\hat{F}_{\delta}$ & 0.10027390 & 0.25064280 & 0.49995950 & 0.76271710 \\
\hline & & & $\hat{\sigma}_{\delta}$ & $(0.015127)$ & $(0.020992)$ & $(0.031381)$ & $(0.046096)$ \\
\hline & & & $\hat{F}_{c o r}$ & 0.10654790 & 0.25419280 & 0.50291780 & 0.77004410 \\
\hline & & & $\hat{\sigma}_{c o r}$ & $(0.036520)$ & $(0.040110)$ & $(0.048113)$ & $(0.069755)$ \\
\hline \multirow[t]{6}{*}{1000} & 0.7 & 0.9 & $\hat{F}_{\gamma}$ & 0.15917900 & 0.25006630 & 0.40024530 & 0.56329450 \\
\hline & & & $\hat{\sigma}_{\gamma}$ & $(0.018643)$ & $(0.020021)$ & $(0.028863)$ & $(0.049218)$ \\
\hline & & & $\hat{F}_{\delta}$ & 0.10027390 & 0.25064280 & 0.49995950 & 0.76271710 \\
\hline & & & $\hat{\sigma}_{\delta}$ & $(0.015127)$ & $(0.020992)$ & $(0.031381)$ & $(0.046096)$ \\
\hline & & & $\hat{F}_{c o r}$ & 0.10172020 & 0.25013120 & 0.50040830 & 0.76850170 \\
\hline & & & $\hat{\sigma}_{c o r}$ & $(0.029982)$ & $(0.033366)$ & $(0.048105)$ & $(0.078315)$ \\
\hline \multirow[t]{6}{*}{1000} & 0.9 & 0.9 & $\hat{F}_{\gamma}$ & 0.18074520 & 0.30090130 & 0.50057440 & 0.71202080 \\
\hline & & & $\hat{\sigma}_{\gamma}$ & $(0.019636)$ & $(0.021987)$ & $(0.030532)$ & $(0.046797)$ \\
\hline & & & $\hat{F}_{\delta}$ & 0.10027390 & 0.25064280 & 0.49995950 & 0.76271710 \\
\hline & & & $\hat{\sigma}_{\delta}$ & $(0.015127)$ & $(0.020992)$ & $(0.031381)$ & $(0.046096)$ \\
\hline & & & $\hat{F}_{c o r}$ & 0.10273380 & 0.25113250 & 0.50071790 & 0.76408160 \\
\hline & & & $\hat{\sigma}_{c o r}$ & $(0.024098)$ & $(0.027483)$ & $(0.038165)$ & $(0.057915)$ \\
\hline
\end{tabular}




\section{Apêndice 2}

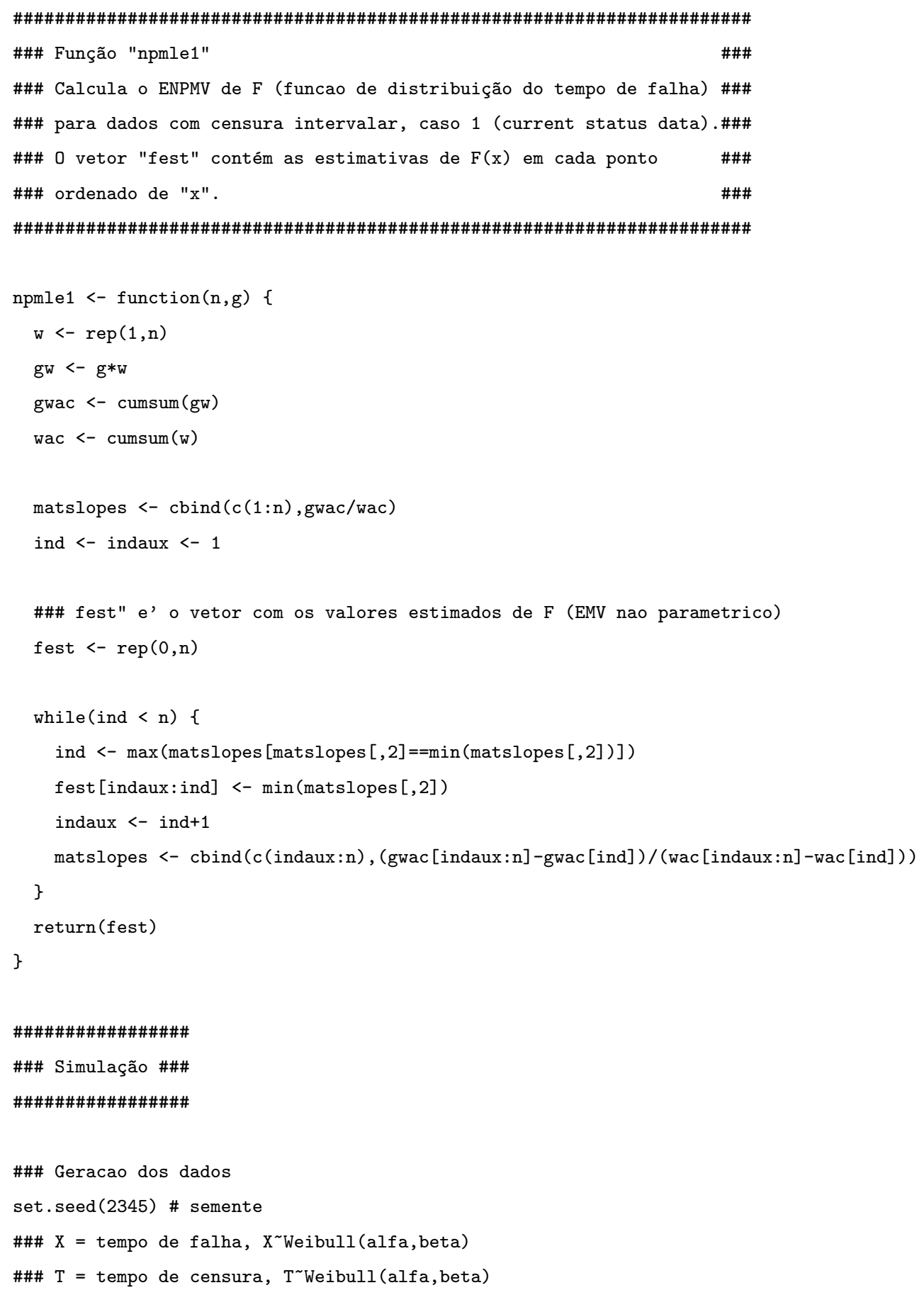




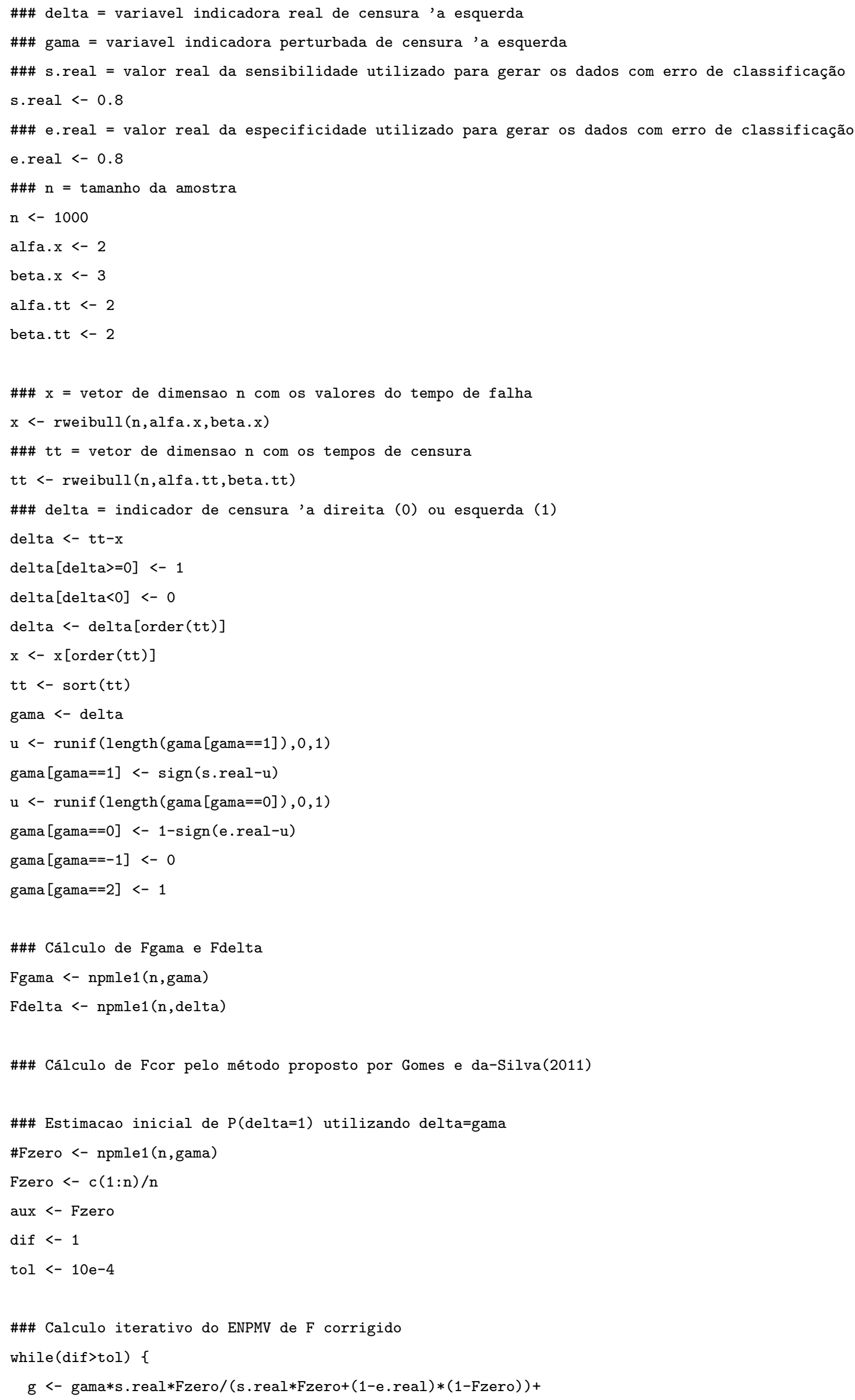




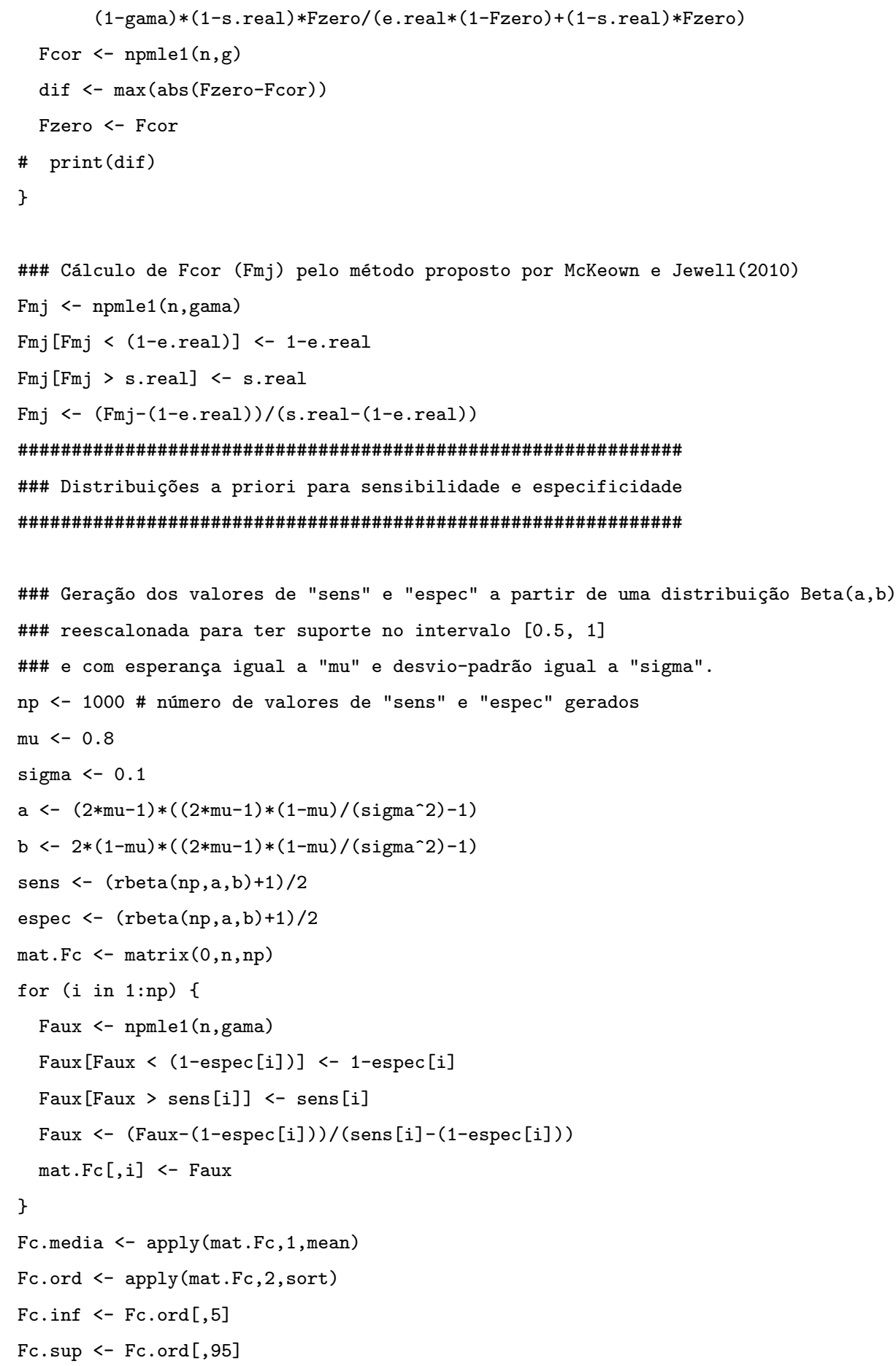

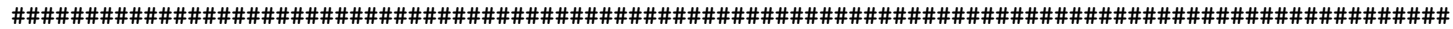

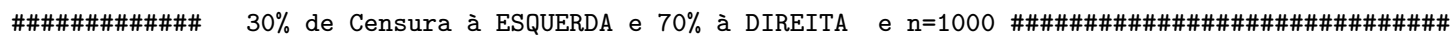

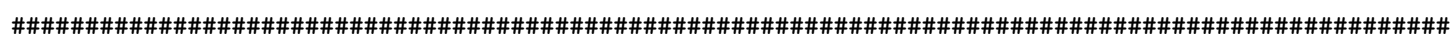

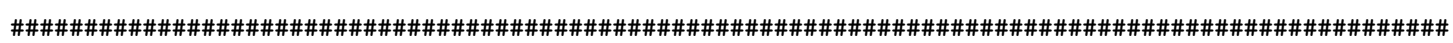
\#\#\#\# Simulação para estimação da esperança e erro-padrão de Fgama, Fdelta e Fcor não suavizadas\#\#

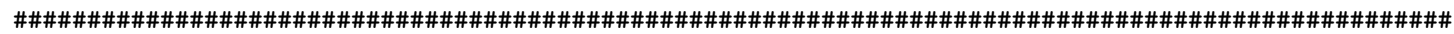

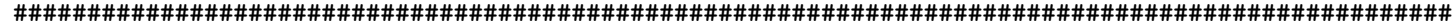
set.seed(2345) \# semente

s.real $<-0.9$ 


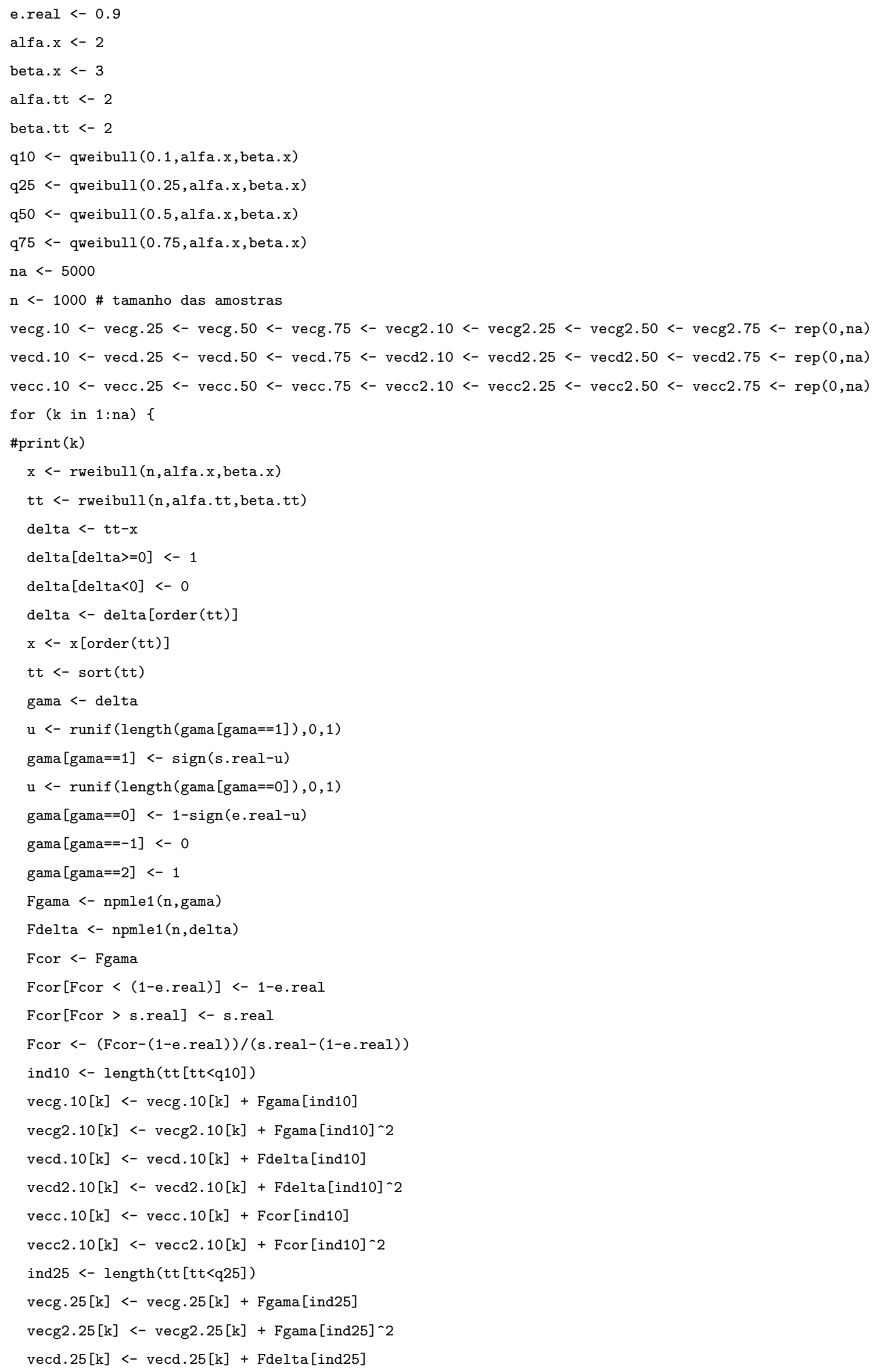




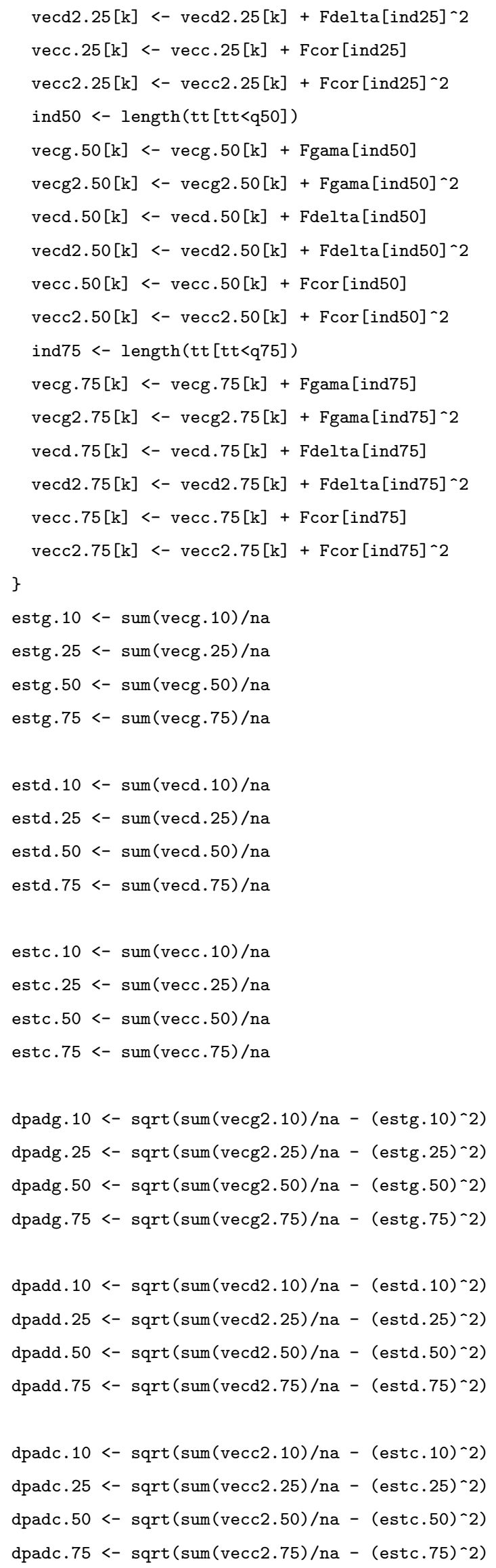




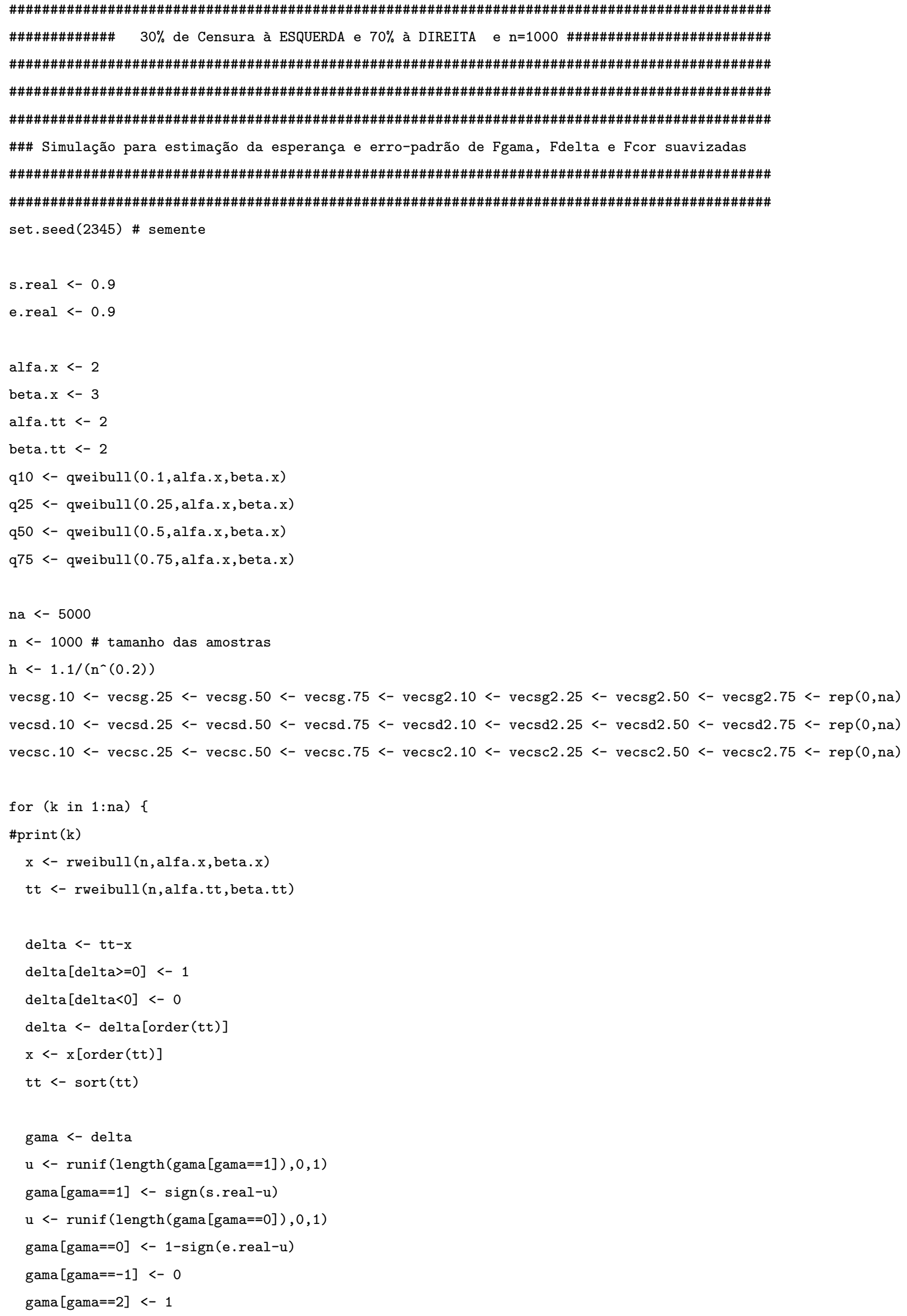




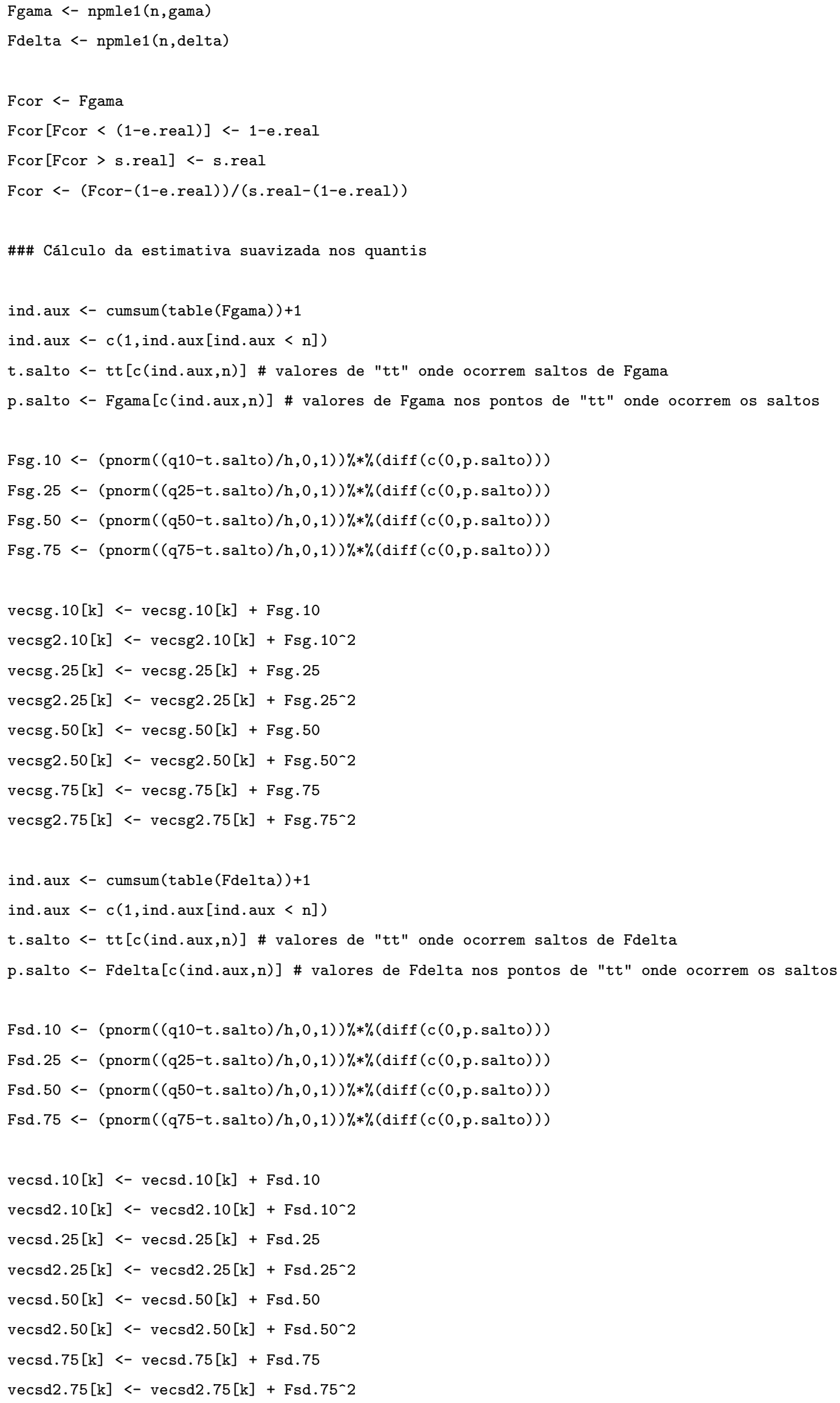




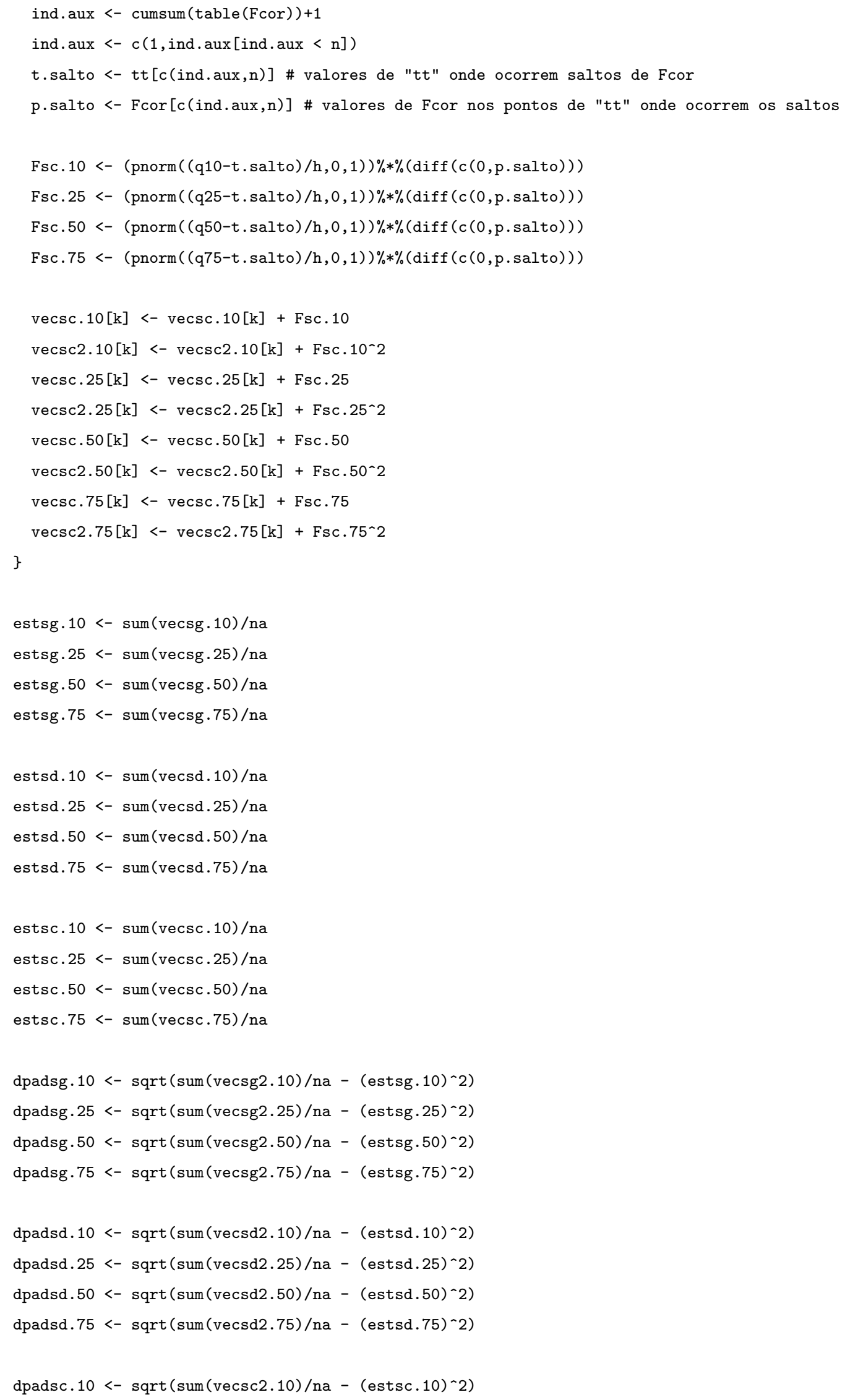


dpadsc. $25<-\operatorname{sqrt}(\operatorname{sum}(\operatorname{vecsc} 2.25) /$ na $-($ estsc.25)^2)

dpadsc.50<- $\operatorname{sqrt}(\operatorname{sum}(\operatorname{vecsc} 2.50) /$ na $-(\operatorname{estsc.50)\leadsto 2)}$

dpadsc. $75<-\operatorname{sqrt}(\operatorname{sum}(\operatorname{vecsc} 2.75) /$ na $-($ estsc.75)^2) 